\title{
Intramolecular Aromatic Nucleophilic Substitution of the Benzimidazole- Activated Nitro Group
}

\author{
Tomasz Fekner, Judith C. Gallucci, and Michael K. Chan
}

\author{
Supporting Information
}

$\underline{\text { Table of Contents }}$

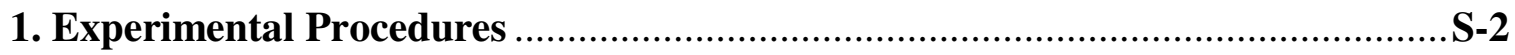

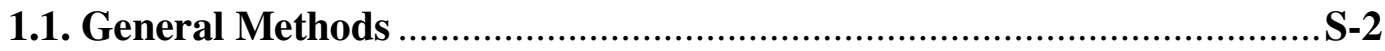

1.2. Synthesis of 2-Aryl-1 $H$-benzimidazoles ……………………….................

1.3. Epoxide-Ring Opening with 2-Aryl-1H-benzimidazoles..........................S-8

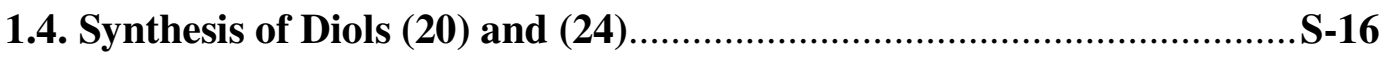

1.5. Intramolecular $S_{N} A r$ Reactions with Monoalcohols .............................S-22

1.6. Intramolecular $S_{N} A r$ Reactions with Diols ...........................................32

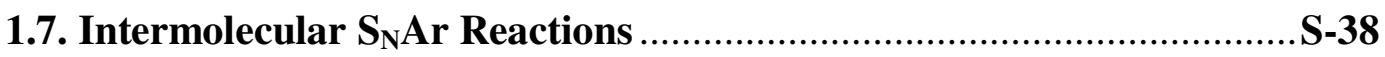

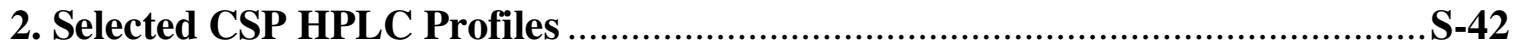

3. X-Ray Single-Crystal Data for Benzimidazole (9) ……………...........................48

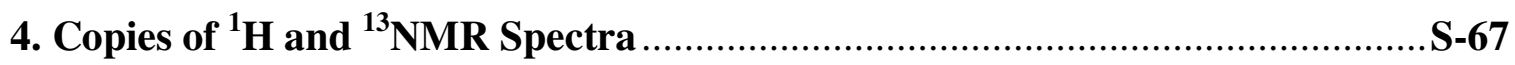




\section{Experimental Procedures}

\subsection{General Methods}

All reactions were performed under anhydrous conditions and an inert atmosphere of argon or nitrogen in the oven-dried glassware with magnetic stirring. Yields refer to chromatographically and spectroscopically ( ${ }^{1} \mathrm{H}$ NMR) homogenous materials, unless otherwise indicated. Reagents were used as obtained from commercial sources, or purified according to the guidelines of Perrin and Armarego. ${ }^{1}$ Evaporation in vacuo refers to the removal of volatiles on a Büchi rotory evaporator attached to an in-house vacuum system $(\sim 20 \mathrm{~mm} \mathrm{Hg})$. Flash chromatography was carried out using Merck Kiesegel $60 \mathrm{~F}_{254}$ (230-400 mesh) silica gel following the method of Still et al. ${ }^{2}$ Only distilled solvents were used as eluents. Thin-layer chromatography (TLC) was performed on Merck DC-Alufolien plates pre-coated with silica gel $60 \mathrm{~F}_{254}$, that were visualized either by quenching of ultraviolet fluorescence, or by charring with $5 \% \mathrm{w} / \mathrm{v}$ phosphomolybdic acid in $95 \% \mathrm{EtOH}, 10 \% \mathrm{w} / \mathrm{v}$ ammonium molybdate in $1 \mathrm{M} \mathrm{H}_{2} \mathrm{SO}_{4}$, or $10 \% \mathrm{KMnO}_{4}$ in $1 \mathrm{M} \mathrm{H}_{2} \mathrm{SO}_{4}$. Observed retention factors $\left(R_{f}\right)$ are quoted to the nearest 0.05 . All reaction solvents were distilled before use, and stored over activated $4 \AA$ molecular sieves, unless otherwise indicated. Anhydrous $\mathrm{CH}_{2} \mathrm{Cl}_{2}$ was obtained by refluxing over $\mathrm{CaH}_{2}$. Anhydrous THF was obtained by distillation, immediately before use, from sodium/benzophenone ketyl under an inert atmosphere of nitrogen. Anhydrous DMF was obtained by distillation under reduced pressure from $\mathrm{CaH}_{2}$, and stored over $4 \AA$ molecular sieves. Petroleum ether refers to the fraction of light petroleum boiling between 40 and $60{ }^{\circ} \mathrm{C}$. High-resolution mass spectrometry (HRMS) measurements are valid to $\pm 5 \mathrm{ppm}$. Melting points (mp) are quoted to the nearest $0.5{ }^{\circ} \mathrm{C}$. Elemental analyses were performed by Atlantic Microlab, Inc., Norcross, GA.

\subsection{Synthesis of 2-Aryl-1H-benzimidazoles}

Each of the previously unreported 2-aryl- $1 H$-benzimidazoles used in these studies was prepared from an appropriate benzoic acid and 1,2-diaminobenzene derivative. Thus, the benzoic acid was converted to the corresponding acyl chloride by treatment with oxalyl chloride in $\mathrm{CH}_{2} \mathrm{Cl}_{2}$ in the presence of a catalytic amount of DMF. The crude acyl chloride was used directly to acylate the 1,2-diaminbenzene derivative in $\mathrm{CH}_{2} \mathrm{Cl}_{2}$ in the presence of $\mathrm{Et}_{3} \mathrm{~N}$. The crude $\mathrm{N}$ -

\footnotetext{
${ }^{1}$ Perrin, D. D.; Armarego, W. L. F. Purification of Laboratory Chemicals; Pergamon Press: New York, 1988.

${ }^{2}$ Still, W. C.; Hahn, M.; Mitra, A. J. Org. Chem. 1978, 43, 2923-2925.
} 
monoacyl product was subsequently subjected to dehydrocyclization in boiling glacial AcOH in the presence of $\mathrm{AcONa}$ to give, after chromatographic purification, the required benzimidazole in high overall yield ( $>80 \%$ in each case). In regards to analytical characterization of these benzimidazoles, it should be mentioned that, due to NH-tautomerizm, it was frequently difficult to obtain good quality ${ }^{13} \mathrm{C}$ NMR spectra for these compounds.

\section{4-Methyl-2-(2-nitrophenyl)-1H-benzimidazole (37)}

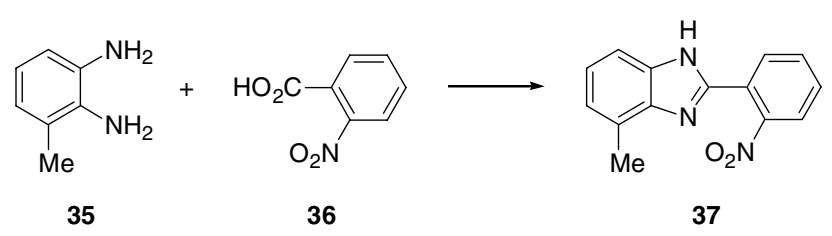

To a suspension of 2-nitrobenzoic acid $36(30.0 \mathrm{~g}, 180 \mathrm{mmol})$ in $\mathrm{CH}_{2} \mathrm{Cl}_{2}(200 \mathrm{~mL})$ was added $(\mathrm{COCl})_{2}(20 \mathrm{~mL}, 0.23 \mathrm{~mol})$, followed by two drops of DMF. The resulting mixture was stirred at $\mathrm{rt}$ for $17 \mathrm{~h}$, and evaporated in vacuo to give a clear oil. The crude acyl chloride was dissolved in $\mathrm{CH}_{2} \mathrm{Cl}_{2}(100 \mathrm{~mL})$, and added dropwise over $1 \mathrm{~h}$ into an ice-cooled solution of 2,3diaminotoluene $35(21.3 \mathrm{~g}, 175 \mathrm{mmol})$ and $\mathrm{Et}_{3} \mathrm{~N}(33 \mathrm{~mL}, 0.23 \mathrm{~mol})$ in $\mathrm{CH}_{2} \mathrm{Cl}_{2}(1.5 \mathrm{~L})$. After an additional $3 \mathrm{~h}$ at $0{ }^{\circ} \mathrm{C} \cdot \mathrm{rt}$, the volatiles were removed in vacuo, and the residue was refluxed in glacial $\mathrm{AcOH}(40 \mathrm{~mL})$ in the presence of AcONa $(14.7 \mathrm{~g}, 175 \mathrm{mmol})$ for $19 \mathrm{~h}$. The reaction mixture was cooled to rt, evaporated in vacuo, and partitioned between $\mathrm{CH}_{2} \mathrm{Cl}_{2}$ and water. The biphasic mixture was cooled in an ice bath, and neutralized with solid $\mathrm{K}_{2} \mathrm{CO}_{3}$ during vigorous stirring. The phases were separated, and the extraction was completed with additional portions of $\mathrm{CH}_{2} \mathrm{Cl}_{2}$. The combined organic extracts were dried $\left(\mathrm{MgSO}_{4}\right)$, and evaporated in vacuo. Purification by flash chromatography (silica gel, $\mathrm{CH}_{2} \mathrm{Cl}_{2} \cdot \mathrm{CH}_{2} \mathrm{Cl}_{2} / \mathrm{EtOAc}$, 6/1) gave the title compound 37 (40.3 g, 91\%) as a yellow solid: $\mathrm{R}_{f}=0.55\left(\mathrm{CH}_{2} \mathrm{Cl}_{2} / \mathrm{EtOAc}, 3 / 1\right)$; mp 167.0-167.5 ${ }^{\circ} \mathrm{C}\left(\right.$ EtOAc/petroleum ether); ${ }^{1} \mathrm{H}$ NMR $\left(250 \mathrm{MHz}, d_{6}-\mathrm{Me}_{2} \mathrm{CO}\right) 2.56(\mathrm{~s}, 3 \mathrm{H}), 7.05$ (d, J=7.0 Hz, $1 \mathrm{H}), 7.15$ (t, $J=7.5 \mathrm{~Hz}, 1 \mathrm{H}), 7.42(\mathrm{br} \mathrm{d}, J=6.5 \mathrm{~Hz}, 1 \mathrm{H}), 7.67$ (dt, $J=7.5,2.0 \mathrm{~Hz}, 1 \mathrm{H}), 7.73(\mathrm{dt}$, $J=7.5,1.5 \mathrm{~Hz}, 1 \mathrm{H}), 7.90(\sim \mathrm{dd}, J=7.5,2.0 \mathrm{~Hz}, 1 \mathrm{H}), 7.95(\sim \mathrm{dd}, J=7.5,2.0 \mathrm{~Hz}, 1 \mathrm{H})$, and 12.2 (br s, $1 \mathrm{H}) ;{ }^{13} \mathrm{C}\left\{{ }^{1} \mathrm{H}\right\} \mathrm{NMR}\left(101 \mathrm{MHz}, d_{6}\right.$-DMSO) 16.48, 17.00, 109.1, 116.6, 121.6, 122.0, $123.0,123.5,124.3,124.7,124.8,128.8,130.7,131.2,131.3,132.5,132.7,134.3,134.5,143.2$, 143.4, 146.7, 147.3, 148.9, and 149.0; ${ }^{4} \mathrm{IR}\left(\mathrm{CHCl}_{3}\right) v_{\max } 1533,1449$, and $1349 \mathrm{~cm}^{-1}$; MS (ESI) $\mathrm{m} / z$ (rel intensity) $254\left(100 \%, \mathrm{MH}^{+}\right)$and 208 (20); HRMS calcd for $\mathrm{C}_{14} \mathrm{H}_{12} \mathrm{~N}_{3} \mathrm{O}_{2}\left(\mathrm{MH}^{+}\right)$254.0929,

\footnotetext{
${ }^{3}$ Vanelle, P.; Liegeois, C. T.; Meuche, J.; Maldonado, J.; Crozet, M. P. Heterocycles 1997, 45, 955-962.

${ }^{4}$ Most peaks doubled due to the NH-tautomerizm.
} 
found 254.0925; Anal. Calcd for $\mathrm{C}_{16} \mathrm{H}_{13} \mathrm{~N}_{3} \mathrm{O}_{2}$ : C, 66.40; H, 4.38; N, 16.59. Found: C, 66.50; $\mathrm{H}$, $4.37 ; \mathrm{N}, 16.61$.

\section{2-(3-Chloro-2-nitrophenyl)-4-methyl-1H-benzimidazole (39)}



To a suspension of 3-chloro-2-nitrobenzoic acid $38(5.00 \mathrm{~g}, 24.8 \mathrm{mmol})$ in $\mathrm{CH}_{2} \mathrm{Cl}_{2}(20$ $\mathrm{mL})$ was added $(\mathrm{COCl})_{2}(2.8 \mathrm{~mL}, 32 \mathrm{mmol})$, followed by a drop of DMF. After $1 \mathrm{~h}$, the resulting clear solution was evaporated in vacuo to give a white solid. The crude acyl chloride was dissolved in $\mathrm{CH}_{2} \mathrm{Cl}_{2}(50 \mathrm{~mL})$, and added dropwise over $30 \mathrm{~min}$ to a solution of 2,3diaminotoluene $35(2.94 \mathrm{~g}, 24.1 \mathrm{mmol})$ and $\mathrm{Et}_{3} \mathrm{~N}(4.5 \mathrm{~mL}, 32 \mathrm{mmol})$ in $\mathrm{CH}_{2} \mathrm{Cl}_{2}(250 \mathrm{~mL})$ at 0 ${ }^{\circ} \mathrm{C}$. After an additional $2 \mathrm{~h}$ at $0{ }^{\circ} \mathrm{C} \cdot \mathrm{rt}$, the volatiles were removed in vacuo to give a yellow solid. The solid was dissolved in glacial AcOH (50 mL), AcONa (2.03 g, $24.8 \mathrm{mmol})$ was added, and the mixture was refluxed for $13 \mathrm{~h}$. The reaction mixture was cooled to $\mathrm{rt}$, evaporated in vacuo, and partitioned between $\mathrm{CH}_{2} \mathrm{Cl}_{2}$ and water. The biphasic mixture was cooled in an ice bath, and neutralized with solid $\mathrm{K}_{2} \mathrm{CO}_{3}$ during vigorous stirring. The phases were separated, and the extraction was completed with additional portions of $\mathrm{CH}_{2} \mathrm{Cl}_{2}$. The combined organic extracts were dried $\left(\mathrm{MgSO}_{4}\right)$, and evaporated in vacuo. Purification by flash chromatography (silica gel, $\left.\mathrm{CH}_{2} \mathrm{Cl}_{2} \cdot \mathrm{CH}_{2} \mathrm{Cl}_{2} / \mathrm{EtOAc}, 10 / 1\right)$ gave the title compound $39(6.23 \mathrm{~g}, 91 \%)$ as a light yellow solid: $\mathrm{R}_{f}=0.70\left(\mathrm{CH}_{2} \mathrm{Cl}_{2} / \mathrm{EtOAc}, 9 / 1\right) ; \mathrm{mp} 201.5-202.5^{\circ} \mathrm{C}\left(\mathrm{Me}_{2} \mathrm{CO}\right) ;{ }^{1} \mathrm{H} \mathrm{NMR}\left(250 \mathrm{MHz}, d_{6}-\mathrm{Me}_{2} \mathrm{CO}\right)$ $2.55(\mathrm{~s}, 3 \mathrm{H}), 7.06$ (d, $J=7.0 \mathrm{~Hz}, 1 \mathrm{H}), 7.16$ (t, $J=7.5 \mathrm{~Hz}, 1 \mathrm{H}), 7.42$ (d, $J=7.5 \mathrm{~Hz}, 1 \mathrm{H}), 7.72$ (t, $J$ $=8.0 \mathrm{~Hz}, 1 \mathrm{H}), 7.77(\mathrm{dd}, J=8.0,2.0 \mathrm{~Hz}, 1 \mathrm{H}), 8.07(\mathrm{dd}, J=7.0,2.0 \mathrm{~Hz}, 1 \mathrm{H})$, and $12.0(\mathrm{br} \mathrm{s}, 1 \mathrm{H})$; IR (KBr) $v_{\max } 1542,1441$, and $1373 \mathrm{~cm}^{-1}$; MS (ESI) $\mathrm{m} / z$ (rel intensity) $288\left(100 \%, \mathrm{MH}^{+}\right.$) and 242 (35); HRMS calcd for $\mathrm{C}_{14} \mathrm{H}_{11} \mathrm{ClN}_{3} \mathrm{O}_{2}\left(\mathrm{MH}^{+}\right)$288.0540, found 288.0522; Anal. Calcd for $\mathrm{C}_{14} \mathrm{H}_{10} \mathrm{ClN}_{3} \mathrm{O}_{2}: \mathrm{C}, 58.45 ; \mathrm{H}, 3.50 ; \mathrm{Cl}, 12.32 ; \mathrm{N}, 14.61$. Found: C, 58.40; H, 3.44; Cl, 12.43; N, 14.48 .

4-Methyl-2-(3-methyl-2-nitrophenyl)-1H-benzimidazole (41)

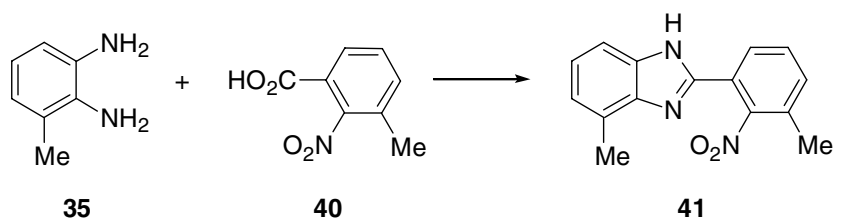


To a suspension of 3-methyl-2-nitrobenzoic acid 40 (5.00 g, $27.6 \mathrm{mmol})$ in $\mathrm{CH}_{2} \mathrm{Cl}_{2}(20$ $\mathrm{mL})$ was added $(\mathrm{COCl})_{2}(2.9 \mathrm{~mL}, 39 \mathrm{mmol})$, followed by a drop of DMF. After $3 \mathrm{~h}$, the resulting clear solution was evaporated in vacuo to give a white solid. The crude acyl chloride was dissolved in $\mathrm{CH}_{2} \mathrm{Cl}_{2}(50 \mathrm{~mL})$, and added dropwise over $30 \mathrm{~min}$ to a solution of 2,3diaminotoluene 35 (3.01 g, $24.6 \mathrm{mmol})$ and $\mathrm{Et}_{3} \mathrm{~N}(4.6 \mathrm{~mL}, 33 \mathrm{mmol})$ in $\mathrm{CH}_{2} \mathrm{Cl}_{2}(300 \mathrm{~mL})$ at 0 ${ }^{\circ} \mathrm{C}$. After an additional $4 \mathrm{~h}$ at $0{ }^{\circ} \mathrm{C} \cdot \mathrm{rt}$, the volatiles were removed in vacuo to give a yellow solid. The solid was dissolved in glacial AcOH (50 mL), AcONa (2.26 g, $27.6 \mathrm{mmol})$ was added, and the mixture was refluxed for $17 \mathrm{~h}$. The reaction mixture was cooled to $\mathrm{rt}$, evaporated in vacuo, and partitioned between $\mathrm{CH}_{2} \mathrm{Cl}_{2}$ and water. The biphasic mixture was cooled in an ice bath, and neutralized with solid $\mathrm{K}_{2} \mathrm{CO}_{3}$ during vigorous stirring. The phases were separated, and the extraction was completed with additional portions of $\mathrm{CH}_{2} \mathrm{Cl}_{2}$. The combined organic extracts were dried $\left(\mathrm{MgSO}_{4}\right)$, and evaporated in vacuo to give a crude product as an off-white solid. Purification by flash chromatography (silica gel, $\mathrm{CH}_{2} \mathrm{Cl}_{2} \cdot \mathrm{CH}_{2} \mathrm{Cl}_{2} / \mathrm{EtOAc}, 30 / 1$ ) gave the title compound 41 (5.85 g, 89\%) as a white solid: $\mathrm{R}_{f}=0.45$ (EtOAc/petroleum ether, 1/1); mp 186.0$187.5{ }^{\circ} \mathrm{C}$ (EtOAc/petroleum ether); ${ }^{1} \mathrm{H}$ NMR (400 MHz, $d_{6}$-DMSO) 2.34 (s, 3H), 2.54 (s, 3H), $7.04(\mathrm{~d}, J=7.0 \mathrm{~Hz}, 1 \mathrm{H}), 7.13(\mathrm{t}, J=7.5 \mathrm{~Hz}, 1 \mathrm{H}), 7.42$ (br s, 1H), 7.60 (d, $J=7.5 \mathrm{~Hz}, 1 \mathrm{H}), 7.69$ $(\mathrm{t}, J=7.5 \mathrm{~Hz}, 1 \mathrm{H})$, and $7.94($ br s, $1 \mathrm{H}) ; \mathrm{IR}\left(\mathrm{CHCl}_{3}\right) v_{\max } 1537$ and $1370 \mathrm{~cm}^{-1}$; MS (ESI) $\mathrm{m} / z$ (rel intensity) $290\left(70 \%, \mathrm{MNa}^{+}\right), 268$ (100), and 222 (45); HRMS calcd for $\mathrm{C}_{15} \mathrm{H}_{13} \mathrm{~N}_{3} \mathrm{NaO}_{2}\left(\mathrm{MNa}^{+}\right)$ 290.0905, found 290.0890; Anal. Calcd for $\mathrm{C}_{15} \mathrm{H}_{13} \mathrm{~N}_{3} \mathrm{O}_{2}: \mathrm{C}, 67.40 ; \mathrm{H}, 4.90 ; \mathrm{N}, 15.72$. Found: C, 67.27; H, 4.86; N, 15.73 .

\section{2-(2-Fluorophenyl)-4-methyl-1H-benzimidazole (43)}

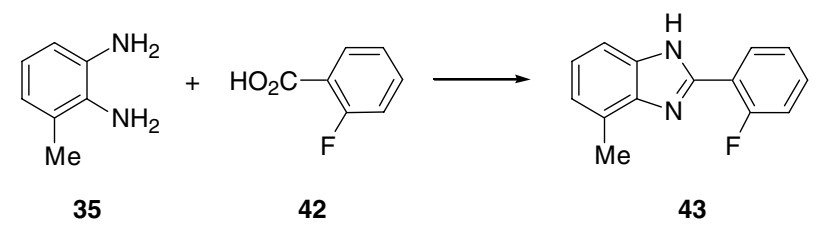

To a suspension of 2-fluorobenzoic acid 42 (5.00 g, $35.7 \mathrm{mmol})$ in $\mathrm{CH}_{2} \mathrm{Cl}_{2}$ (20 mL) was added $(\mathrm{COCl})_{2}(4.1 \mathrm{~mL}, 46 \mathrm{mmol})$, followed by a drop of DMF. The resulting mixture was stirred at $\mathrm{rt}$ for $16 \mathrm{~h}$, and evaporated in vacuo to give a yellow oil. The crude acyl chloride was dissolved in $\mathrm{CH}_{2} \mathrm{Cl}_{2}(50 \mathrm{~mL})$, and added dropwise over $1 \mathrm{~h}$ to a solution of 2,3-diaminotoluene ${ }^{3}$ 35 (4.22 g, $34.6 \mathrm{mmol})$ and $\mathrm{Et}_{3} \mathrm{~N}(6.3 \mathrm{~mL}, 45 \mathrm{mmol})$ in $\mathrm{CH}_{2} \mathrm{Cl}_{2}(250 \mathrm{~mL})$ at $0{ }^{\circ} \mathrm{C}$. After an additional $2 \mathrm{~h}$ at $0{ }^{\circ} \mathrm{C} \cdot \mathrm{rt}$, the volatiles were removed in vacuo, and the residue was refluxed in glacial $\mathrm{AcOH}(50 \mathrm{~mL})$ in the presence of $\mathrm{AcONa}(2.93 \mathrm{~g}, 35.7 \mathrm{mmol})$ for $8 \mathrm{~h}$. The reaction 
mixture was cooled to rt, evaporated in vacuo, and partitioned between $\mathrm{CH}_{2} \mathrm{Cl}_{2}$ and water. The biphasic mixture was cooled in an ice bath, and neutralized with solid $\mathrm{K}_{2} \mathrm{CO}_{3}$ during vigorous stirring. The phases were separated, and the extraction was completed with additional portions of $\mathrm{CH}_{2} \mathrm{Cl}_{2}$. The combined organic extracts were dried $\left(\mathrm{MgSO}_{4}\right)$, and evaporated in vacuo to give an orange foam. Purification by flash chromatography (silica gel, $\mathrm{CH}_{2} \mathrm{Cl}_{2} \cdot \mathrm{CH}_{2} \mathrm{Cl}_{2} / \mathrm{EtOAc}, 20 / 1$ ) gave the title compound $43(6.80 \mathrm{~g}, 87 \%)$ as a white solid: $\mathbf{R}_{f}=0.45$ (EtOAc/petroleum ether, 3/1); mp 161.0-161.5 ${ }^{\circ} \mathrm{C}$ (EtOAc/petroleum ether); ${ }^{1} \mathrm{H}$ NMR (250 MHz, $\left.\mathrm{CDCl}_{3}\right) \quad 2.54$ (s, 3H), $6.97(\mathrm{~d}, J=7.0 \mathrm{~Hz}, 1 \mathrm{H}), 7.03-7.17(\mathrm{~m}, 2 \mathrm{H}), 7.19$ (d, $J=7.5 \mathrm{~Hz}, 1 \mathrm{H}), 7.22-7.46(\mathrm{~m}, 2 \mathrm{H}), 8.40$ (dt, $J=8.0,2.0 \mathrm{~Hz}, 1 \mathrm{H})$, and $9.78(\mathrm{br} \mathrm{s}, 1 \mathrm{H})$; IR $\left(\mathrm{CHCl}_{3}\right) v_{\max } 1466 \mathrm{~cm}^{-1}$; MS (ESI) $\mathrm{m} / z$ (rel intensity) $227\left(100 \%, \mathrm{MH}^{+}\right)$; HRMS calcd for $\mathrm{C}_{14} \mathrm{H}_{12} \mathrm{FN}_{2}\left(\mathrm{MH}^{+}\right)$227.0984, found 227.0987; Anal. Calcd for $\mathrm{C}_{14} \mathrm{H}_{11} \mathrm{FN}_{2}: \mathrm{C}, 74.32 ; \mathrm{H}, 4.90 ; \mathrm{N}, 12.38$. Found: C, 74.06; H, 4.88; N, 12.36.

\section{2-(2-Chlorophenyl)-4-methyl-1H-benzimidazole (45)}

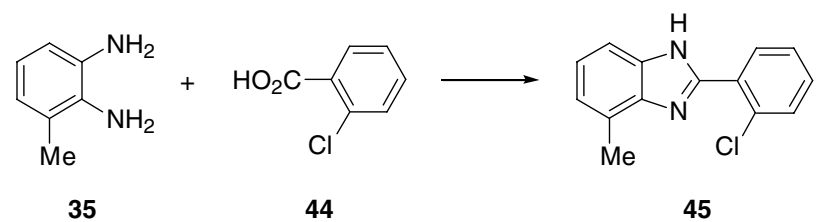

To a suspension of 2-chlorobenzoic acid 44 (5.00 g, $31.9 \mathrm{mmol})$ in $\mathrm{CH}_{2} \mathrm{Cl}_{2}$ (30 mL) was added $(\mathrm{COCl})_{2}(3.6 \mathrm{~mL}, 42 \mathrm{mmol})$, followed by a drop of DMF. The resulting mixture was stirred at $\mathrm{rt}$ for $90 \mathrm{~min}$, and evaporated in vacuo to give a clear oil. The crude acyl chloride was dissolved in $\mathrm{CH}_{2} \mathrm{Cl}_{2}(50 \mathrm{~mL})$, and added dropwise over $40 \mathrm{~min}$ to a solution of 2,3diaminotoluene $35(3.78 \mathrm{~g}, 30.9 \mathrm{mmol})$ and $\mathrm{Et}_{3} \mathrm{~N}(5.8 \mathrm{~mL}, 42 \mathrm{mmol})$ in $\mathrm{CH}_{2} \mathrm{Cl}_{2}(250 \mathrm{~mL})$ at 0 ${ }^{\circ} \mathrm{C}$. After an additional $3 \mathrm{~h}$ at $0{ }^{\circ} \mathrm{C} \cdot \mathrm{rt}$, the volatiles were removed in vacuo to give a pale brown oil. The residue was refluxed in glacial $\mathrm{AcOH}(50 \mathrm{~mL})$ in the presence of $\mathrm{AcONa}(2.62 \mathrm{~g}, 31.9$ $\mathrm{mmol}$ ) for $15 \mathrm{~h}$. The reaction mixture was cooled to $\mathrm{rt}$, evaporated in vacuo, and partitioned between $\mathrm{CH}_{2} \mathrm{Cl}_{2}$ and water. The biphasic mixture was cooled in an ice bath, and neutralized with solid $\mathrm{K}_{2} \mathrm{CO}_{3}$ during vigorous stirring. The phases were separated, and the extraction was completed with additional portions of $\mathrm{CH}_{2} \mathrm{Cl}_{2}$. The combined organic extracts were dried $\left(\mathrm{MgSO}_{4}\right)$, and evaporated in vacuo to give a yellow foam. Purification by flash chromatography ( silica gel, petroleum ether - petroleum ether/EtOAc, 3/1) gave the title compound 45 (6.80 g, $88 \%$ ) as a white foam: $\mathrm{R}_{f}=0.35$ (petroleum ether/EtOAc, 3/1); ${ }^{1} \mathrm{H}$ NMR $\left(250 \mathrm{MHz}, \mathrm{CDCl}_{3}\right.$ ) $2.54(\mathrm{~s}, 3 \mathrm{H}), 6.98(\sim \mathrm{d}, J=7.0 \mathrm{~Hz}, 1 \mathrm{H}), 7.08(\mathrm{t}, J=7.5 \mathrm{~Hz}, 1 \mathrm{H}), 7.19-7.26(\mathrm{~m}, 2 \mathrm{H}), 7.29-7.38(\mathrm{~m}$, $2 \mathrm{H}), 8.11-8.15(\mathrm{~m}, 1 \mathrm{H})$, and 9.70 (br s, 1H); IR $\left(\mathrm{CHCl}_{3}\right) v_{\max } 1449,1396$, and $1045 \mathrm{~cm}^{-1}$; MS 
(ESI) $\mathrm{m} / \mathrm{z}$ (rel intensity) $265\left(30 \%, \mathrm{MNa}^{+}\right)$and 243(100); HRMS calcd for $\mathrm{C}_{14} \mathrm{H}_{11} \mathrm{ClN}_{2} \mathrm{Na}\left(\mathrm{MNa}^{+}\right)$ 265.0508 , found 265.0510 .

\section{2-(2-Methoxyphenyl)-1H-benzimidazole (48)}



To a solution of 2-methoxybenzoic acid $47(5.00 \mathrm{~g}, 32.9 \mathrm{mmol})$ in $\mathrm{CH}_{2} \mathrm{Cl}_{2}(30 \mathrm{~mL})$ was added $(\mathrm{COCl})_{2}(3.7 \mathrm{~mL}, 43 \mathrm{mmol})$, followed by a drop of DMF. After $16 \mathrm{~h}$ at $\mathrm{rt}$, the resulting clear solution was evaporated in vacuo to give a pale yellow oil. The crude acyl chloride was dissolved in $\mathrm{CH}_{2} \mathrm{Cl}_{2}(50 \mathrm{~mL})$, and added dropwise over $30 \mathrm{~min}$ to an ice-cooled solution of 1,2diaminobenzene 46 (3.89 g, $31.9 \mathrm{mmol})$ and $\mathrm{Et}_{3} \mathrm{~N}(6.0 \mathrm{~mL}, 43 \mathrm{mmol})$ in $\mathrm{CH}_{2} \mathrm{Cl}_{2}(250 \mathrm{~mL})$. After an additional $3 \mathrm{~h}$ at $0{ }^{\circ} \mathrm{C} \cdot \mathrm{rt}$, the volatiles were removed in vacuo to give a brown solid. The solid was dissolved in glacial AcOH $(50 \mathrm{~mL})$, AcONa $(2.7 \mathrm{~g}, 33 \mathrm{mmol})$ was added, and the mixture was refluxed for $19 \mathrm{~h}$. The reaction mixture was cooled to rt, evaporated in vacuo, and partitioned between $\mathrm{CH}_{2} \mathrm{Cl}_{2}$ and water. The biphasic mixture was cooled in an ice bath, and neutralized with solid $\mathrm{K}_{2} \mathrm{CO}_{3}$ during vigorous stirring. The phases were separated, and the extraction was completed with additional portions of $\mathrm{CH}_{2} \mathrm{Cl}_{2}$. The combined organic extracts were dried $\left(\mathrm{MgSO}_{4}\right)$, and evaporated in vacuo to give a crude product as a yellow solid. Purification by flash chromatography (silica gel, $\mathrm{CH}_{2} \mathrm{Cl}_{2} \cdot \mathrm{CH}_{2} \mathrm{Cl}_{2} / \mathrm{EtOAc}$, 8/1) gave the title compound 48 (7.10 g, 93\%) as a white solid: $\mathrm{R}_{f}=0.60\left(\mathrm{CH}_{2} \mathrm{Cl}_{2} / \mathrm{EtOAc}, 3 / 1\right) ; \mathrm{mp} 179.5-180.5^{\circ} \mathrm{C}$ (EtOAc/petroleum ether) (Lit. $\left.{ }^{5} 181{ }^{\circ} \mathrm{C}\right) ;{ }^{1} \mathrm{H}$ NMR $\left(250 \mathrm{MHz}, \mathrm{CDCl}_{3}\right) \quad 3.95$ (s, 3H), 6.49 (d, $J=$ $8.5 \mathrm{~Hz}, 1 \mathrm{H}$ ), 7.03 (dt, $J=7.5,1.0 \mathrm{~Hz}, 1 \mathrm{H}), 7.12-7.20$ (m, 2H), 7.30 (ddd, $J=8.5,7.5,2.0 \mathrm{~Hz}$, 1H), 7.54 (br s, 2H), $8.49(\mathrm{dd}, J=8.0,2.0 \mathrm{~Hz}, 1 \mathrm{H})$, and $10.6(\mathrm{br} \mathrm{s}, 1 \mathrm{H}) ;{ }^{13} \mathrm{C}\left\{{ }^{1} \mathrm{H}\right\} \mathrm{NMR}(63 \mathrm{MHz}$, $\left.\mathrm{CDCl}_{3}\right) \quad 55.92,111.5,117.9,121.7,122.5,130.2,131.2,149.9$, and 156.8; $\mathrm{IR}\left(\mathrm{CHCl}_{3}\right) v_{\text {max }} 1472$, 1441,1279 , and $1240 \mathrm{~cm}^{-1}$; MS (ESI) $\mathrm{m} / z$ (rel intensity) $225\left(100 \%, \mathrm{MH}^{+}\right)$; HRMS calcd for $\mathrm{C}_{14} \mathrm{H}_{13} \mathrm{~N}_{2} \mathrm{O}\left(\mathrm{MH}^{+}\right)$225.1028, found 225.1020.

\footnotetext{
${ }^{5}$ Nardi, D.; Tajana, A.; Rossi, S. J. Het. Chem. 1973, 10, 815-819.
} 


\section{2-(3-Chloro-2-nitrophenyl)-1H-benzimidazole (49)}

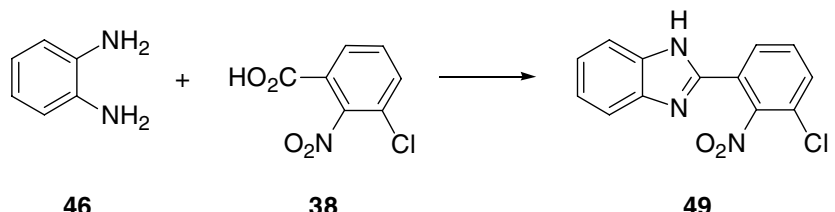

To a suspension of 3-chloro-2-nitrobenzoic acid 38 (5.00 g, $24.8 \mathrm{mmol})$ in $\mathrm{CH}_{2} \mathrm{Cl}_{2}(20$ $\mathrm{mL})$ was added $(\mathrm{COCl})_{2}(2.8 \mathrm{~mL}, 32 \mathrm{mmol})$, followed by a drop of DMF. After $1 \mathrm{~h}$, the resulting clear solution was evaporated in vacuo to give a white solid. The crude acyl chloride was dissolved in $\mathrm{CH}_{2} \mathrm{Cl}_{2}(50 \mathrm{~mL})$, and added dropwise over $1 \mathrm{~h}$ to a solution of 1,2-diaminobenzene $46(2.60 \mathrm{~g}, 24.1 \mathrm{mmol})$ and $\mathrm{Et}_{3} \mathrm{~N}(4.5 \mathrm{~mL}, 32 \mathrm{mmol})$ in $\mathrm{CH}_{2} \mathrm{Cl}_{2}(250 \mathrm{~mL})$ at $0{ }^{\circ} \mathrm{C}$. After an additional $2 \mathrm{~h}$ at $0{ }^{\circ} \mathrm{C} \rightarrow \mathrm{rt}$, the volatiles were removed in vacuo to give a pale yellow solid. The solid was dissolved in glacial AcOH (50 mL), AcONa (2.03 g, $24.8 \mathrm{mmol})$ was added, and the mixture was refluxed for $19 \mathrm{~h}$. The reaction mixture was cooled to $\mathrm{rt}$, evaporated in vacuo, and partitioned between $\mathrm{CH}_{2} \mathrm{Cl}_{2}$ and water. The biphasic mixture was cooled in an ice bath, and neutralized with solid $\mathrm{K}_{2} \mathrm{CO}_{3}$ during vigorous stirring. The phases were separated, and the extraction was completed with additional portions of $\mathrm{CH}_{2} \mathrm{Cl}_{2}$. The combined organic extracts were dried $\left(\mathrm{MgSO}_{4}\right)$, and evaporated in vacuo to give a crude product as a creamy solid. Purification by flash chromatography (silica gel, $\mathrm{CH}_{2} \mathrm{Cl}_{2} \rightarrow \mathrm{CH}_{2} \mathrm{Cl}_{2} / \mathrm{EtOAc}$, 2/1) gave the title compound 49 (5.78 g, 88\%) as an off-white solid: $\mathrm{R}_{f}=0.70\left(\right.$ EtOAc); mp $>260{ }^{\circ} \mathrm{C}\left(\mathrm{Me}{ }_{2} \mathrm{CO}\right) ;{ }^{1} \mathrm{H}$ NMR (400 MHz, $d_{6}$-DMSO) $\delta$ 7.22-7.30 (m, 2H), 7.58-7.67 (m, 2H), $7.81(\mathrm{t}, J=8.0 \mathrm{~Hz}, 1 \mathrm{H})$, $7.88(\mathrm{~d}, J=8.0 \mathrm{~Hz}, 1 \mathrm{H}), 8.13(\mathrm{~d}, J=7.5 \mathrm{~Hz}, 1 \mathrm{H})$, and 13.3 (br s, 1H); IR (KBr) $v_{\max } 1541,1450$, 1440, 1415, and $1376 \mathrm{~cm}^{-1}$; MS (ESI) $\mathrm{m} / z$ (rel intensity) $274\left(100 \%, \mathrm{MH}^{+}\right.$); HRMS calcd for $\mathrm{C}_{13} \mathrm{H}_{9} \mathrm{ClN}_{3} \mathrm{O}_{2}\left(\mathrm{MH}^{+}\right)$274.0383, found 274.0372; Anal. Calcd for $\mathrm{C}_{13} \mathrm{H}_{8} \mathrm{ClN}_{3} \mathrm{O}_{2}$ : C, 57.05; $\mathrm{H}$, 2.95; Cl, 12.95; N, 15.35. Found: C, 56.85; H, 2.86; Cl, 13.13; N, 15.27.

\subsection{Epoxide-Ring Opening with 2-Aryl-1H-benzimidazoles}

The free NH group of 2-aryl-1H-benzimidazoles, and in particular 2-(2-nitrophenyl)-1Hbenzimidazoles, is poorly nucleophilic, so its reaction with epoxides is not very facile. The $\mathrm{Cu}(\mathrm{OTf})_{2}$-catalyzed reaction used in these studies to prepare the desired alcohols does not usually go to completion. With a few notable exceptions, the levels of conversions are usually low $(<50 \%)$. In general, 4-substituted 2-aryl-1H-benzimidazoles are far better substrates than their unsubstituted counterparts. It presumably stems from a significantly reduced ability, due to steric 
reasons, to form $\mathrm{Cu}$ (II) complexes by the substrates (and the products derived from them) that are heavily substituted in the vicinity of the benzimidazole nitrogen. For the unsubstituted benzimidazoles, in turn, the catalytic cycle ceases to operate relatively quickly, as an active $\mathrm{Cu}$ (II) species gets converted into various unreactive complexes. On a few occasions, we attempted to use stochiometric amounts of $\mathrm{Cu}(\mathrm{OTf})_{2}$, but it did not lead to improved yields of the alcohols. As far as epoxide substrates are concerned, it was found that only terminal epoxides are reactive enough, with the 2-monosubstitued derivatives being significantly better substrates than the 2,2-disubstitued ones.

\section{1-[4-Methyl-2-(2-nitrophenyl)benzimidazol-1-yl]propan-2-ol (4)}

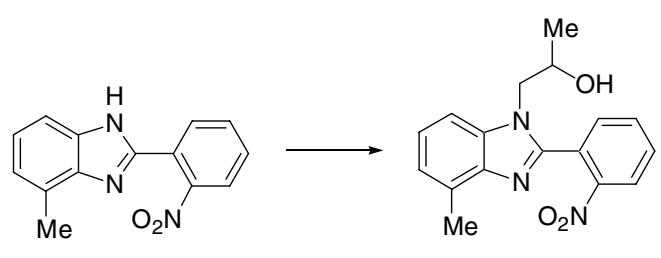

37

4

To a solution of benzimidazole $37(1.27 \mathrm{~g}, 5.00 \mathrm{mmol})$ in $\mathrm{MeCN}(8 \mathrm{~mL})$ were added propylene oxide $(2.9 \mathrm{~g}, 50 \mathrm{mmol})$ and $\mathrm{Cu}(\mathrm{OTf})_{2}(362 \mathrm{mg}, 1.00 \mathrm{mmol})$. After $22 \mathrm{~h}$ at $40{ }^{\circ} \mathrm{C}$, the reaction mixture was cooled to $\mathrm{rt}$ and evaporated in vacuo. The residue was partitioned between $\mathrm{CH}_{2} \mathrm{Cl}_{2}$ and satd $\mathrm{NaHCO}_{3}$, and stirred at $\mathrm{rt}$ for $30 \mathrm{~min}$. The phases were separated, and the extraction was completed with additional portions of $\mathrm{CH}_{2} \mathrm{Cl}_{2}$. The combined organic extracts were dried $\left(\mathrm{MgSO}_{4}\right)$, and evaporated in vacuo to give a brown oil. Purification by flash chromatography (silica gel, $\mathrm{CH}_{2} \mathrm{Cl}_{2} \rightarrow \mathrm{CH}_{2} \mathrm{Cl}_{2} / \mathrm{EtOAc}, 4 / 1$ ) gave the recovered starting material 37 (670 mg, 53\%) and the title compound 4 (499 mg, 32\%) as a pale yellow solid. Alcohol 4: $\mathrm{R}_{f}$ $=0.55$ (EtOAc); mp 171.0-172.0 ${ }^{\circ} \mathrm{C}$ (EtOAc/petroleum ether); ${ }^{1} \mathrm{H}$ NMR $\left(400 \mathrm{MHz}, \mathrm{CDCl}_{3}\right) \delta$ $1.13(\mathrm{~d}, J=6.0 \mathrm{~Hz}, 3 \mathrm{H}), 2.66(\mathrm{~s}, 4 \mathrm{H}), 3.95(\mathrm{dd}, J=14.5,8.5 \mathrm{~Hz}, 1 \mathrm{H}), 4.03(\mathrm{dd}, J=14.5,3.5 \mathrm{~Hz}$, $1 \mathrm{H}), 4.10-4.20(\mathrm{~m}, 1 \mathrm{H}), 7.15(\mathrm{~d}, J=6.5 \mathrm{~Hz}, 1 \mathrm{H}), 7.23-7.34(\mathrm{~m}, 2 \mathrm{H}), 7.64(\mathrm{dd}, J=7.0,1.5 \mathrm{~Hz}$, $1 \mathrm{H}), 7.68(\mathrm{dd}, J=8.0,1.5 \mathrm{~Hz}, 1 \mathrm{H}), 7.73(\mathrm{dt}, J=7.5,1.0 \mathrm{~Hz}, 1 \mathrm{H})$, and $8.16(\mathrm{dd}, J=8.0,1.0 \mathrm{~Hz}$, $1 \mathrm{H}) ;{ }^{13} \mathrm{C}\left\{{ }^{1} \mathrm{H}\right\} \mathrm{NMR}\left(101 \mathrm{MHz}, \mathrm{CDCl}_{3}\right) \delta 16.65,20.63,52.03,65.98,107.8,123.0,123.2,124.9$, 126.3, 130.1, 130.9, 132.9, 133.2, 134.6, 142.2, 148.6, and 149.0; IR $\left(\mathrm{CHCl}_{3}\right) v_{\max } 1534,1458$, 1397, and $1348 \mathrm{~cm}^{-1}$; MS (ESI) $\mathrm{m} / z$ (rel intensity) $312\left(100 \%, \mathrm{MH}^{+}\right.$); HRMS calcd for $\mathrm{C}_{17} \mathrm{H}_{18} \mathrm{~N}_{3} \mathrm{O}_{3}\left(\mathrm{MH}^{+}\right)$312.1348, found 312.1328; Anal. Calcd for $\mathrm{C}_{17} \mathrm{H}_{17} \mathrm{~N}_{3} \mathrm{O}_{3}$ : C, 65.58; H, 5.50; N, 13.50. Found: C, 65.49; H, 5.45; N, 13.39. 


\section{1-[4-Methyl-2-(2-nitrophenyl)benzimidazol-1-yl]hexan-2-ol (6)}

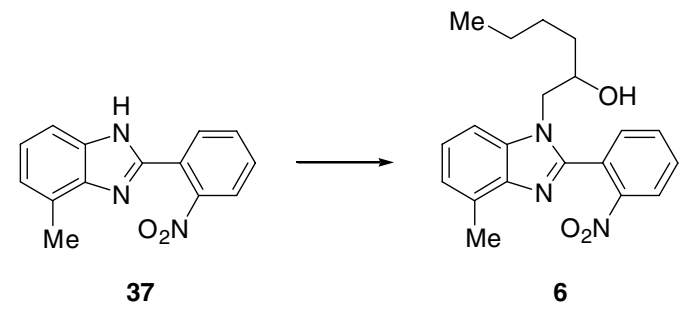

To a solution of benzimidazole 37 (1.27 g, $5.00 \mathrm{mmol})$ in MeCN (8 mL) were added 1,2epoxyhexane $(1.5 \mathrm{~g}, 15 \mathrm{mmol})$ and $\mathrm{Cu}(\mathrm{OTf})_{2}(362 \mathrm{mg}, 1.00 \mathrm{mmol})$. After $20 \mathrm{~h}$ at reflux, the reaction mixture was cooled to $\mathrm{rt}$ and evaporated in vacuo. The residue was partitioned between $\mathrm{CH}_{2} \mathrm{Cl}_{2}$ and satd $\mathrm{NaHCO}_{3}$, and stirred at $\mathrm{rt}$ for $30 \mathrm{~min}$. The phases were separated, and the extraction was completed with additional portions of $\mathrm{CH}_{2} \mathrm{Cl}_{2}$. The combined organic extracts were dried $\left(\mathrm{MgSO}_{4}\right)$, and evaporated in vacuo to give a brown oil. Purification by flash chromatography (silica gel, $\mathrm{CH}_{2} \mathrm{Cl}_{2} \rightarrow \mathrm{CH}_{2} \mathrm{Cl}_{2} / \mathrm{EtOAc}$, 4/1) gave the recovered starting material $37(329 \mathrm{mg}, 26 \%)$ and the title compound $6(754 \mathrm{mg}, 43 \%)$ as a yellow solid. Alcohol 6: $\mathrm{R}_{f}=$ 0.65 (EtOAc); ${ }^{1} \mathrm{H}$ NMR (400 MHz, $\left.\mathrm{CDCl}_{3}\right) \delta 0.87$ (t, $\left.J=6.5 \mathrm{~Hz}, 3 \mathrm{H}\right), 1.15-1.49(\mathrm{~m}, 6 \mathrm{H}), 2.66$ (s, $3 \mathrm{H}), 3.87-3.96(\mathrm{~m}, 2 \mathrm{H}), 4.07(\mathrm{dd}, J=18.0,7.0 \mathrm{~Hz}, 1 \mathrm{H}), 7.15(\mathrm{~d}, J=6.5 \mathrm{~Hz}, 1 \mathrm{H}), 7.23-7.33(\mathrm{~m}$, $2 \mathrm{H}), 7.60-7.73(\mathrm{~m}, 3 \mathrm{H})$, and $8.15(\mathrm{~d}, J=7.5 \mathrm{~Hz}, 1 \mathrm{H}) ;{ }^{13} \mathrm{C}\left\{{ }^{1} \mathrm{H}\right\} \mathrm{NMR}\left(63 \mathrm{MHz}, \mathrm{CDCl}_{3}\right) \delta 14.32$, 17.16, 22.89, 27.89, 34.81, 51.49, 70.38, 108.3, 123.5, 123.7, 125.3, 126.7, 130.5, 131.4, 133.5, 133.7, 135.1, 142.6, 149.2, and 149.4; IR $\left(\mathrm{CHCl}_{3}\right) v_{\max }$ 2960, 1535, 1458, 1397, 1348, and 1242 $\mathrm{cm}^{-1}$; MS (ESI) $m / z$ (rel intensity) 354 (100\%, $\mathrm{MH}^{+}$) and 254 (15); HRMS calcd for $\mathrm{C}_{20} \mathrm{H}_{24} \mathrm{~N}_{3} \mathrm{O}_{3}$ $\left(\mathrm{MH}^{+}\right)$354.1817, found 354.1830.

\section{3,3-Dimethyl-1-[2-(2-nitrophenyl)benzimidazol-1-yl]butan-2-ol (8)}

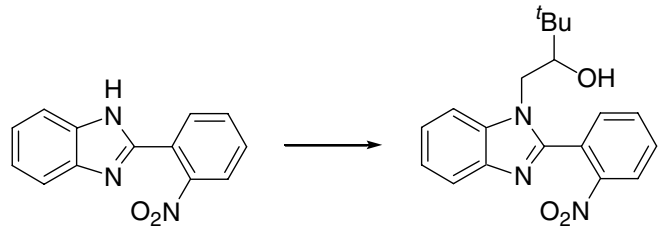

50

8

To a suspension of benzimidazole $\mathbf{5 0}^{6}$ (2.39 g, $\left.10.0 \mathrm{mmol}\right)$ in $\mathrm{MeCN}$ (40 mL) were added 3,3-dimethyl-1,2-epoxybutane $(5.0 \mathrm{~g}, 50 \mathrm{mmol})$ and $\mathrm{Cu}(\mathrm{OTf})_{2}(724 \mathrm{mg}, 2.00 \mathrm{mmol})$. After $17 \mathrm{~h}$ at reflux, the reaction mixture was cooled to $\mathrm{rt}$ and evaporated in vacuo. The residue was partitioned between $\mathrm{CH}_{2} \mathrm{Cl}_{2}$ and satd $\mathrm{NaHCO}_{3}$, and stirred at $\mathrm{rt}$ for $30 \mathrm{~min}$. The phases were

\footnotetext{
${ }^{6}$ Zaika, L. L.; Joullié, M. M. J. Het. Chem. 1966, 3, 289-298.
} 
separated, and the extraction was completed with additional portions of $\mathrm{CH}_{2} \mathrm{Cl}_{2}$. The combined organic extracts were dried $\left(\mathrm{MgSO}_{4}\right)$, and evaporated in vacuo to give a brown foam. Purification by flash chromatography (silica gel, $\mathrm{CH}_{2} \mathrm{Cl}_{2} \rightarrow \mathrm{CH}_{2} \mathrm{Cl}_{2} / \mathrm{EtOAc}$, 4/1) gave the title compound 8 (603 mg, $18 \%$ ) as a white solid: $\mathrm{R}_{f}=0.70$ (EtOAc); $\mathrm{mp} \mathrm{191.5-193.0}{ }^{\circ} \mathrm{C}$ (EtOAc/petroleum ether); ${ }^{1} \mathrm{H}$ NMR $\left(400 \mathrm{MHz}, \mathrm{CDCl}_{3}\right) \delta 0.90(\mathrm{~s}, 9 \mathrm{H}), 2.58$ (br s, $\left.1 \mathrm{H}\right), 3.61(\mathrm{~d}, J=10.0 \mathrm{~Hz}$, 1H), 3.96 (dd, $J=14.5,10.0 \mathrm{~Hz}, 1 \mathrm{H}), 4.24(\mathrm{~d}, J=14.5 \mathrm{~Hz}, 1 \mathrm{H}), 7.32$ (t, $J=6.5 \mathrm{~Hz}, 1 \mathrm{H}), 7.36$ (t, $J=7.0 \mathrm{~Hz}, 1 \mathrm{H}), 7.62-7.74(\mathrm{~m}, 4 \mathrm{H})$, and $8.15(\mathrm{~d}, J=8.0 \mathrm{~Hz}, 1 \mathrm{H}) ;{ }^{13} \mathrm{C}\left\{{ }^{1} \mathrm{H}\right\} \mathrm{NMR}(63 \mathrm{MHz}$, $\left.\mathrm{CDCl}_{3}\right) \delta 25.86,34.72,47.56,77.97,110.9,120.5,122.9,123.6,125.1,126.6,131.3,133.4$, 133.5, 135.5, 143.4, 149.5, and 150.1; IR $\left(\mathrm{CHCl}_{3}\right) v_{\max } 2965,1534,1458,1404$, and $1349 \mathrm{~cm}^{-1}$; MS (ESI) $\mathrm{m} / \mathrm{z}$ (rel intensity) $340\left(100 \%, \mathrm{MH}^{+}\right.$) and 240 (10); HRMS calcd for $\mathrm{C}_{19} \mathrm{H}_{21} \mathrm{~N}_{3} \mathrm{NaO}_{3}$ $\left(\mathrm{MNa}^{+}\right)$362.1481, found 362.1473; Anal. Calcd for $\mathrm{C}_{19} \mathrm{H}_{21} \mathrm{~N}_{3} \mathrm{O}_{3}: \mathrm{C}, 67.24 ; \mathrm{H}, 6.24 ; \mathrm{N}, 12.38$. Found: C, 67.14; H, 6.25; N, 12.31 .

\section{3,3-Dimethyl-1-[4-methyl-2-(2-nitrophenyl)benzimidazol-1-yl]butan-2-ol (10)}

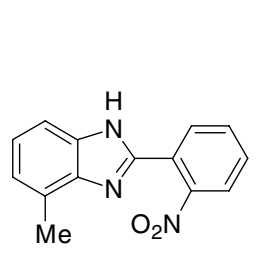

37

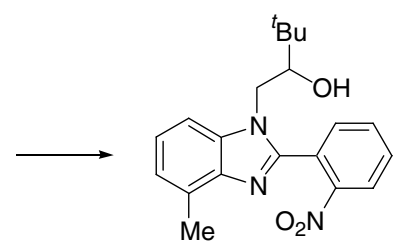

10

To a solution of benzimidazole $37(2.00 \mathrm{~g}, 7.91 \mathrm{mmol})$ and 3,3-dimethyl-1,2-epoxybutane (3.95 g, $39.5 \mathrm{mmol})$ in $\mathrm{MeCN}(40 \mathrm{~mL})$ was added $\mathrm{Cu}(\mathrm{OTf})_{2}(573 \mathrm{mg}, 1.58 \mathrm{mmol})$. The reaction mixture was refluxed for $24 \mathrm{~h}$, cooled to $\mathrm{rt}$, and evaporated in vacuo. The residue was partitioned between $\mathrm{CH}_{2} \mathrm{Cl}_{2}$ and satd $\mathrm{NaHCO}_{3}$, and stirred at $\mathrm{rt}$ for $30 \mathrm{~min}$. The phases were separated, and the extraction was completed with additional portions of $\mathrm{CH}_{2} \mathrm{Cl}_{2}$. The combined organic extracts were dried $\left(\mathrm{MgSO}_{4}\right)$, and evaporated in vacuo to give a brown solid. Purification by flash chromatography (silica gel, $\mathrm{CH}_{2} \mathrm{Cl}_{2} \rightarrow \mathrm{CH}_{2} \mathrm{Cl}_{2} / \mathrm{EtOAc}$, 2/1) gave the recovered starting material $37(780 \mathrm{mg}, 39 \%)$ and the title compound $10(1.39 \mathrm{~g}, 50 \%)$ as a pale yellow solid. Alcohol 10: $\mathrm{R}_{f}=0.70$ (EtOAc); mp 203.5-204.5 ${ }^{\circ} \mathrm{C}$ (EtOAc/petroleum ether); ${ }^{1} \mathrm{H}$ NMR (250 $\left.\mathrm{MHz}, \mathrm{CDCl}_{3}\right) \delta 0.83(\mathrm{~s}, 9 \mathrm{H}), 2.01(\mathrm{~d}, J=4.0 \mathrm{~Hz}, 1 \mathrm{H}), 2.61(\mathrm{~s}, 3 \mathrm{H}), 3.56(\mathrm{dd}, J=10.0,4.0 \mathrm{~Hz}$, $1 \mathrm{H}), 3.87(\mathrm{dd}, J=14.5,10.0 \mathrm{~Hz}, 1 \mathrm{H}), 4.18(\mathrm{~d}, J=14.5 \mathrm{~Hz}, 1 \mathrm{H}), 7.07(\mathrm{~d}, J=7.0 \mathrm{~Hz}, 1 \mathrm{H}), 7.19(\mathrm{t}$, $J=8.0 \mathrm{~Hz}, 1 \mathrm{H}), 7.26(\mathrm{~d}, J=8.0 \mathrm{~Hz}, 1 \mathrm{H}), 7.57-7.72(\mathrm{~m}, 3 \mathrm{H})$, and $8.10(\sim \mathrm{dd}, J=8.0,1.0 \mathrm{~Hz}, 1 \mathrm{H})$;

${ }^{13} \mathrm{C}\left\{{ }^{1} \mathrm{H}\right\}$ NMR $\left(101 \mathrm{MHz}, \mathrm{CDCl}_{3}\right) \delta 16.69,25.41,34.22,47.17,77.60,107.8,122.9,123.1$, 
124.8, 126.5, 130.2, 130.8, 133.0, 133.1, 134.7, 142.4, 148.8, and 149.2; IR $\left(\mathrm{CHCl}_{3}\right) v_{\max } 2964$, 1534, 1458, 1398, and $1348 \mathrm{~cm}^{-1}$; MS (ESI) $\mathrm{m} / z$ (rel intensity) $354\left(100 \%, \mathrm{MH}^{+}\right)$and $254(10)$; HRMS calcd for $\mathrm{C}_{20} \mathrm{H}_{23} \mathrm{~N}_{3} \mathrm{NaO}_{3}\left(\mathrm{MNa}^{+}\right)$376.1637, found 376.1644; Anal. Calcd for $\mathrm{C}_{20} \mathrm{H}_{23} \mathrm{~N}_{3} \mathrm{O}_{3}$ : C, 67.97; H, 6.56; N, 11.89. Found: C, 67.97; H, 6.61; N, 11.91 .

\section{2-Methyl-1-[4-methyl-2-(2-nitrophenyl)benzimidazol-1-yl]propan-2-ol (14)}

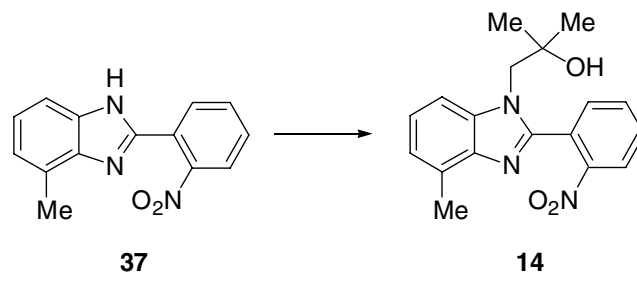

To a solution of benzimidazole $37(1.27 \mathrm{~g}, 5.0 \mathrm{mmol})$ and 1,2-epoxy-2-methylpropane $(1.8 \mathrm{~g}, 25 \mathrm{mmol})$ in $\mathrm{MeCN}(8 \mathrm{~mL})$ was added $\mathrm{Cu}(\mathrm{OTf})_{2}(362 \mathrm{mg}, 1.00 \mathrm{mmol})$ to give a darkbrown suspension. The reaction mixture was refluxed for $24 \mathrm{~h}$, cooled to $\mathrm{rt}$, and evaporated in vacuo. The residue was partitioned between $\mathrm{CH}_{2} \mathrm{Cl}_{2}$ and satd $\mathrm{NaHCO}_{3}$, and stirred at $\mathrm{rt}$ for $1 \mathrm{~h}$. The phases were separated, and the extraction was completed with additional portions of $\mathrm{CH}_{2} \mathrm{Cl}_{2}$. The combined organic extracts were dried $\left(\mathrm{MgSO}_{4}\right)$, and evaporated in vacuo to give a brown oil. Purification by flash chromatography (silica gel, $\mathrm{CH}_{2} \mathrm{Cl}_{2} \rightarrow \mathrm{CH}_{2} \mathrm{Cl}_{2} / \mathrm{EtOAc}$, 10/1; repurification: silica gel, $\left.\mathrm{CH}_{2} \mathrm{Cl}_{2} / \mathrm{EtOAc}, 2 / 1\right)$ gave the recovered starting material 37 (1.08 g, 85\%) and the title compound $\mathbf{1 4}(197 \mathrm{mg}, 12 \%)$ as a pale yellow solid: $\mathrm{R}_{f}=0.55$ (EtOAc); ${ }^{1} \mathrm{H}$ NMR (400 MHz, CDCl $) \delta 1.12$ (s, 6H), 1.98 (s, 1H), 2.64 (s, 3H), 4.08 (s, 2H), 7.09 (d, J = 7.5 $\mathrm{Hz}, 1 \mathrm{H}), 7.21(\mathrm{t}, J=7.5 \mathrm{~Hz}, 1 \mathrm{H}), 7.31(\mathrm{~d}, J=9.0 \mathrm{~Hz}, 1 \mathrm{H}), 7.61(\mathrm{dd}, J=7.5,1.0 \mathrm{~Hz}, 1 \mathrm{H}), 7.64(\mathrm{t}$, $J=7.5 \mathrm{~Hz}, 1 \mathrm{H}), 7.64(\mathrm{dt}, J=8.0,1.5 \mathrm{~Hz}, 1 \mathrm{H}), 7.72(\mathrm{dt}, J=7.5,1.5 \mathrm{~Hz}, 1 \mathrm{H})$, and $8.12(\mathrm{dd}, J=$ 8.0, $1.0 \mathrm{~Hz}, 1 \mathrm{H}) ;{ }^{13} \mathrm{C}\left\{{ }^{1} \mathrm{H}\right\} \operatorname{NMR}\left(101 \mathrm{MHz}, \mathrm{CDCl}_{3}\right) \delta 16.67,27.69,55.07,71.99,108.7,122.9$, 123.2, 125.0, 127.1, 130.2, 130.7, 132.8, 133.1, 135.7, 142.3, 148.8, and 149.1; IR $\left(\mathrm{CHCl}_{3}\right) v_{\max }$ 1533, 1456, and $1348 \mathrm{~cm}^{-1}$; MS (ESI) $\mathrm{m} / z$ (rel intensity) $326\left(100 \%, \mathrm{MH}^{+}\right), 308(20)$, and 254 (70); HRMS calcd for $\mathrm{C}_{18} \mathrm{H}_{19} \mathrm{~N}_{3} \mathrm{NaO}_{3}\left(\mathrm{MNa}^{+}\right) 348.1324$, found 348.1318. 


\section{1-[2-(3-Chloro-2-nitrophenyl)-4-methylbenzimidazol-1-yl]-3,3-dimethylbutan-2-ol (16)}

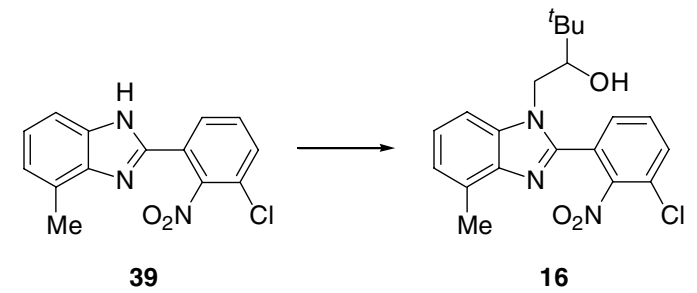

To a solution of benzimidazole 39 (2.00 $\mathrm{g}, 7.04 \mathrm{mmol})$ and 3,3-dimethyl-1,2epoxybutane $(3.5 \mathrm{~g}, 35 \mathrm{mmol})$ in $\mathrm{MeCN}(50 \mathrm{~mL})$ was added $\mathrm{Cu}(\mathrm{OTf})_{2}(510 \mathrm{mg}, 1.41 \mathrm{mmol})$. The reaction mixture was refluxed for $22 \mathrm{~h}$, cooled to $\mathrm{rt}$, and evaporated in vacuo. The residue was partitioned between $\mathrm{CH}_{2} \mathrm{Cl}_{2}$ and satd $\mathrm{NaHCO}_{3}$, and stirred at $\mathrm{rt}$ for $30 \mathrm{~min}$. The phases were separated, and the extraction was completed with additional portions of $\mathrm{CH}_{2} \mathrm{Cl}_{2}$. The combined organic extracts were dried $\left(\mathrm{MgSO}_{4}\right)$, and evaporated in vacuo to give a brown oil. Purification by flash chromatography (silica gel, $\mathrm{CH}_{2} \mathrm{Cl}_{2} \rightarrow$ EtOAc) gave the recovered starting material 39 $(1.31 \mathrm{~g}, 66 \%)$ and the title compound $16(554 \mathrm{mg}, 20 \%)$ as a white solid. Alcohol 16: $\mathrm{R}_{f}=0.70$ $\left(\mathrm{CH}_{2} \mathrm{Cl}_{2} / \mathrm{EtOAc}, 2 / 1\right)$; mp 235.5-237.0 ${ }^{\circ} \mathrm{C}\left(\right.$ EtOAc); ${ }^{1} \mathrm{H} \mathrm{NMR}\left(250 \mathrm{MHz}, \mathrm{CDCl}_{3}\right) \delta 0.94(\mathrm{~s}, 9 \mathrm{H})$, $1.97(\mathrm{~d}, J=4.5 \mathrm{~Hz}, 1 \mathrm{H}), 2.63$ (s, 3H), 3.65 (ddd, $J=10.0,4.5,1.5 \mathrm{~Hz}, 1 \mathrm{H}), 4.05$ (dd, $J=14.5$, $10.0 \mathrm{~Hz}, 1 \mathrm{H}), 4.33$ (dd, $J=14.5,1.5 \mathrm{~Hz}, 1 \mathrm{H}), 7.05-7.28(\mathrm{~m}, 3 \mathrm{H}), 7.53(\mathrm{t}, J=7.5 \mathrm{~Hz}, 1 \mathrm{H}), 7.64$ $(\mathrm{d}, J=8.0 \mathrm{~Hz}, 1 \mathrm{H})$, and $7.69(\mathrm{~d}, J=7.0 \mathrm{~Hz}, 1 \mathrm{H}) ;{ }^{13} \mathrm{C}\left\{{ }^{1} \mathrm{H}\right\} \mathrm{NMR}\left(101 \mathrm{MHz}, d_{6}-\mathrm{DMSO}\right) \delta 16.14$, 25.48, 34.41, 46.76, 75.40, 109.1, 122.3, 123.0, 124.6, 125.5, 129.0, 131.3, 131.8, 132.0, 134.9, 141.9, 146.3, and 148.8; IR $\left(\mathrm{CHCl}_{3}\right) v_{\max } 2965,1546,1457$, and $1365 \mathrm{~cm}^{-1}$; MS (ESI) $\mathrm{m} / z$ (rel intensity) $388\left(100 \%, \mathrm{MH}^{+}\right)$and $288(10)$; $\mathrm{HRMS}$ calcd for $\mathrm{C}_{20} \mathrm{H}_{22} \mathrm{ClN}_{3} \mathrm{NaO}_{3}\left(\mathrm{MNa}^{+}\right) 410.1247$, found 410.1234 .

\section{3,3-Dimethyl-1-[4-methyl-2-(3-methyl-2-nitrophenyl)benzimidazol-1-yl]butan-2-ol (18)}

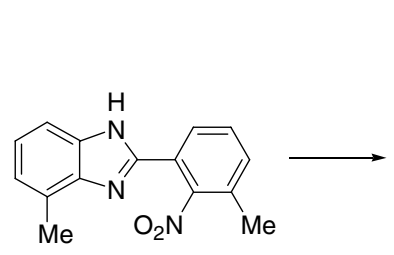

41

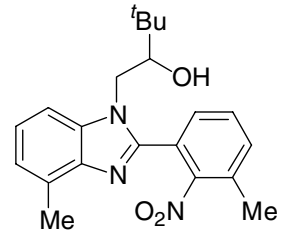

18

To a solution of benzimidazole 41 (2.00 g, $7.5 \mathrm{mmol})$ in MeCN (15 mL) were added 3,3dimethyl-1,2-epoxybutane $(3.0 \mathrm{~g}, 30 \mathrm{mmol})$ and $\mathrm{Cu}(\mathrm{OTf})_{2}(543 \mathrm{mg}, 1.5 \mathrm{mmol})$ to give a darkbrown solution. After $19 \mathrm{~h}$ at reflux, the reaction mixture was cooled to $\mathrm{rt}$ and evaporated in vacuo. The residue was partitioned between $\mathrm{CH}_{2} \mathrm{Cl}_{2}$ and satd $\mathrm{NaHCO}_{3}$, and stirred at $\mathrm{rt}$ for 30 
min. The phases were separated, and the extraction was completed with additional portions of $\mathrm{CH}_{2} \mathrm{Cl}_{2}$. The combined organic extracts were dried $\left(\mathrm{MgSO}_{4}\right)$, and evaporated in vacuo to give a brown foam. Purification by flash chromatography (silica gel, $\mathrm{CH}_{2} \mathrm{Cl}_{2} \rightarrow \mathrm{CH}_{2} \mathrm{Cl}_{2} / \mathrm{EtOAc}$, 4/1) gave the recovered starting material 41 (247 mg, 12\%) and the title compound $\mathbf{1 8}(1.08 \mathrm{~g}, 39 \%)$ as a white solid. Alcohol 18: $\mathrm{R}_{f}=0.55\left(\mathrm{CH}_{2} \mathrm{Cl}_{2} / \mathrm{EtOAc}, 3 / 1\right) ; \mathrm{mp} 216.0-217.5^{\circ} \mathrm{C}$ (EtOAc/petroleum ether); ${ }^{1} \mathrm{H}$ NMR (400 MHz, $d_{6}$-DMSO) $\delta 0.86$ (s, 9H), 2.40 (s, 3H), 2.51 (s, 3H), 3.59 (dd, $J$ $=10.5,5.5 \mathrm{~Hz}, 1 \mathrm{H}), 3.95(\mathrm{dd}, J=14.0,10.5 \mathrm{~Hz}, 1 \mathrm{H}), 4.27(\mathrm{~d}, J=14.0 \mathrm{~Hz}, 1 \mathrm{H}), 5.12(\mathrm{~d}, J=5.5$ $\mathrm{Hz}, 1 \mathrm{H}), 7.06(\mathrm{~d}, J=7.0 \mathrm{~Hz}, 1 \mathrm{H}), 7.21(\mathrm{t}, J=7.5 \mathrm{~Hz}, 1 \mathrm{H}), 7.44(\mathrm{~d}, J=8.0 \mathrm{~Hz}, 1 \mathrm{H}), 7.69$ (dd, $J=$ 8.0, 1.0 Hz, 1H), $7.71(\mathrm{t}, J=7.5 \mathrm{~Hz}, 1 \mathrm{H})$, and $7.99(\mathrm{dd}, J=7.0,1.0 \mathrm{~Hz}, 1 \mathrm{H}) ;{ }^{13} \mathrm{C}\left\{{ }^{1} \mathrm{H}\right\} \mathrm{NMR}(101$ MHz, $d_{6}$-DMSO) $\delta 16.18,17.28,25.47,34.37,46.73,75.61,109.1,122.0,122.5,124.0,128.7$, 130.1, $130.4(2 \times \mathrm{C}), 133.0,134.9,141.9,147.9$, and 150.8; IR $\left(\mathrm{CHCl}_{3}\right) v_{\max } 2964,1535,1457$, and $1365 \mathrm{~cm}^{-1}$; MS (ESI) $\mathrm{m} / z$ (rel intensity) $390\left(75 \%, \mathrm{MNa}^{+}\right), 368$ (100), and 268 (5); HRMS calcd for $\mathrm{C}_{21} \mathrm{H}_{25} \mathrm{~N}_{3} \mathrm{NaO}_{3}\left(\mathrm{MNa}^{+}\right)$390.1794, found 390.1805; Anal. Calcd for $\mathrm{C}_{21} \mathrm{H}_{25} \mathrm{~N}_{3} \mathrm{O}_{3}: \mathrm{C}$, 68.64; H, 6.86; N, 11.44. Found: C, 68.64; H, 6.86; N, 11.30 .

\section{1-[2-(2-Fluorophenyl)-4-methylbenzimidazol-1-yl]-3,3-dimethylbutan-2-ol (28)}

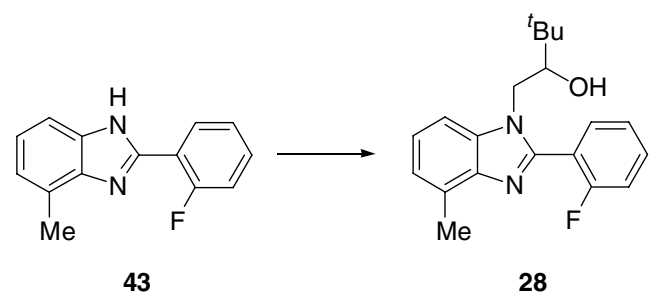

To a solution of benzimidazole $43(2.00 \mathrm{~g}, 8.85 \mathrm{mmol})$ and 3,3-dimethyl-1,2epoxybutane $(4.4 \mathrm{~g}, 44 \mathrm{mmol})$ in $\mathrm{MeCN}(50 \mathrm{~mL})$ was added $\mathrm{Cu}(\mathrm{OTf})_{2}(641 \mathrm{mg}, 1.77 \mathrm{mmol})$. The reaction mixture was refluxed for $16 \mathrm{~h}$, cooled to $\mathrm{rt}$, and evaporated in vacuo. The residue was partitioned between $\mathrm{CH}_{2} \mathrm{Cl}_{2}$ and satd $\mathrm{NaHCO}_{3}$, and stirred at $\mathrm{rt}$ for $30 \mathrm{~min}$. The phases were separated, and the extraction was completed with additional portions of $\mathrm{CH}_{2} \mathrm{Cl}_{2}$. The combined organic extracts were dried $\left(\mathrm{MgSO}_{4}\right)$, and evaporated in vacuo to give a brown oil. Purification by flash chromatography (silica gel, $\mathrm{CH}_{2} \mathrm{Cl}_{2} \rightarrow$ EtOAc) gave the recovered starting material $\mathbf{4 3}$ $(1.40 \mathrm{~g}, 70 \%)$ and the title compound $\mathbf{2 8}(298 \mathrm{mg}, 10 \%)$ as a white solid. Alcohol 28: $\mathrm{R}_{f}=0.70$ (EtOAc/ $\left.\mathrm{CH}_{2} \mathrm{Cl}_{2}, 1 / 1\right)$; mp 178.5-179.0 ${ }^{\circ} \mathrm{C}$ (EtOAc/petroleum ether); ${ }^{1} \mathrm{H}$ NMR (400 MHz, $\mathrm{CDCl}_{3}$ ) $\delta 0.89$ (s, 9H), 1.76 (s, 1H), 2.74 (s, 3H), 3.49 (d, $J=10.0 \mathrm{~Hz}, 1 \mathrm{H}), 4.06$ (dd, $J=14.5,10.0 \mathrm{~Hz}$, $1 \mathrm{H}), 4.31(\mathrm{~d}, J=14.5 \mathrm{~Hz}, 1 \mathrm{H}), 7.15(\mathrm{~d}, J=7.0 \mathrm{~Hz}, 1 \mathrm{H}), 7.19-7.33(\mathrm{~m}, 3 \mathrm{H}), 7.37$ (d, $J=8.0 \mathrm{~Hz}$, 
1H), $7.52(\mathrm{dd}, J=13.0,7.0 \mathrm{~Hz}, 1 \mathrm{H})$, and $7.67(\mathrm{t}, J=7.0 \mathrm{~Hz}, 1 \mathrm{H}) ;{ }^{13} \mathrm{C}\left\{{ }^{1} \mathrm{H}\right\} \mathrm{NMR}(101 \mathrm{MHz}$, $\left.\mathrm{CDCl}_{3}\right) \delta 16.76,25.29,34.23$, [46.6 (d, $\left.J=3.0 \mathrm{~Hz}\right)$ ?], 78.31, 108.0, $115.8(\mathrm{~d}, J=21.5 \mathrm{~Hz}), 119.2$ (d, $J=14.5 \mathrm{~Hz}), 122.7,122.9,124.5(\mathrm{~d}, J=3.5 \mathrm{~Hz}), 130.1,131.9$ (d, $J=8.0 \mathrm{~Hz}), 132.7$ (d, $J=$ $2.0 \mathrm{~Hz}), 135.1,142.3,148.3$, and $160.0(\mathrm{~d}, J=249 \mathrm{~Hz}) ; \mathrm{IR}\left(\mathrm{CHCl}_{3}\right) v_{\max } 2964,1642,1459$, and $1391 \mathrm{~cm}^{-1}$; MS (ESI) $\mathrm{m} / z$ (rel intensity) $327\left(100 \%, \mathrm{MH}^{+}\right.$) and 227 (10); HRMS calcd for $\mathrm{C}_{20} \mathrm{H}_{23} \mathrm{FN}_{2} \mathrm{NaO}\left(\mathrm{MNa}^{+}\right)$349.1692, found 349.1698; Anal. Calcd for $\mathrm{C}_{20} \mathrm{H}_{23} \mathrm{FN}_{2} \mathrm{O}: \mathrm{C}, 73.59 ; \mathrm{H}$, 7.10; N, 8.58. Found: C, 73.50; H, 7.31; N, 8.64.

\section{1-[2-(2-Chlorophenyl)-4-methylbenzimidazol-1-yl]propan-2-ol (29)}

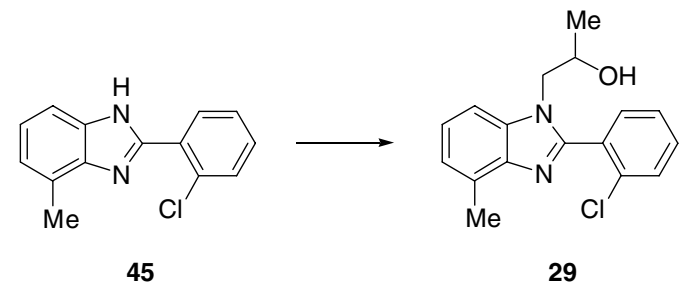

To a solution of benzimidazole 45 (3.00 g, $12.3 \mathrm{mmol})$ in $\mathrm{MeCN}(15 \mathrm{~mL})$ were added propylene oxide $(3.6 \mathrm{~g}, 62 \mathrm{mmol})$ and $\mathrm{Cu}(\mathrm{OTf})_{2}(891 \mathrm{mg}, 2.46 \mathrm{mmol})$ to give a brown suspension. After $20 \mathrm{~h}$ at $65^{\circ} \mathrm{C}$, the reaction mixture was cooled to rt and evaporated in vacuo. The residue was partitioned between $\mathrm{CH}_{2} \mathrm{Cl}_{2}$ and satd $\mathrm{NaHCO}_{3}$, and stirred at $\mathrm{rt}$ for $40 \mathrm{~min}$. The phases were separated, and the extraction was completed with additional portions of $\mathrm{CH}_{2} \mathrm{Cl}_{2}$. The combined organic extracts were dried $\left(\mathrm{MgSO}_{4}\right)$, and evaporated in vacuo to give a light brown foam. Purification by flash chromatography (silica gel, $\mathrm{CH}_{2} \mathrm{Cl}_{2} \rightarrow$ EtOAc) gave the title compound 29 (851 mg, 23\%) as a white solid: $\mathrm{R}_{f}=0.35\left(\mathrm{CH}_{2} \mathrm{Cl}_{2} / \mathrm{EtOAc}, 3 / 1\right)$; mp 164.0-165.0 ${ }^{\circ} \mathrm{C}$ (EtOAc/petroleum ether); ${ }^{1} \mathrm{H}$ NMR $\left(400 \mathrm{MHz}, \mathrm{CDCl}_{3}\right) \delta 1.02(\mathrm{~d}, J=6.0 \mathrm{~Hz}, 3 \mathrm{H}), 2.49$ (br s, 1H), 2.70 (s, 3H), 3.99 (d, $J=6.0 \mathrm{~Hz}, 2 \mathrm{H}$ ), 4.06 (heptet, $J=6.0,6.0 \mathrm{~Hz}, 1 \mathrm{H}$ ), 7.13 (d, $J=7.5 \mathrm{~Hz}$, $1 \mathrm{H}), 7.24(\mathrm{t}, J=7.0 \mathrm{~Hz}, 1 \mathrm{H}), 7.30(\mathrm{~d}, J=8.0 \mathrm{~Hz}, 1 \mathrm{H}), 7.36(\mathrm{dt}, J=7.5,1.0 \mathrm{~Hz}, 1 \mathrm{H}), 7.44(\mathrm{dt}, J=$ $7.5,1.5 \mathrm{~Hz}, 1 \mathrm{H}), 7.50(\mathrm{dd}, J=8.0,1.0 \mathrm{~Hz}, 1 \mathrm{H})$, and $7.53(\mathrm{dd}, J=7.5,1.5 \mathrm{~Hz}, 1 \mathrm{H}) ;{ }^{13} \mathrm{C}\left\{{ }^{1} \mathrm{H}\right\}$ NMR $\left(101 \mathrm{MHz}, \mathrm{CDCl}_{3}\right) \delta 16.76,20.78,51.63,66.20,108.0,122.9(2 \times \mathrm{C}), 126.9,129.6,130.1$, 130.2, 131.2, 132.7, 134.2, 134.6, 142.1, and 150.4; IR $\left(\mathrm{CHCl}_{3}\right) v_{\max } 1607,1453,1391,1335$, and $1241 \mathrm{~cm}^{-1}$; MS (ESI) $\mathrm{m} / z$ (rel intensity) $323\left(35 \%, \mathrm{MNa}^{+}\right.$), 301 (100), and 243 (45); HRMS calcd for $\mathrm{C}_{17} \mathrm{H}_{18} \mathrm{ClN}_{2} \mathrm{O}\left(\mathrm{MNa}^{+}\right)$301.1107, found 301.1112; Anal. Calcd for $\mathrm{C}_{17} \mathrm{H}_{17} \mathrm{ClN}_{2} \mathrm{O}$ : C, 67.88; H, 5.70; Cl, 11.79; N, 9.31. Found: C, 68.02; H, 5.65; Cl, 11.98; N, 9.30. 


\subsection{Synthesis of Diols (20) and (24)}

The diols used in these studies were prepared by the Sharpless asymmetric dihydroxylation reaction (AD) of appropriate $N$-allyl substituted 2-aryl-1H-benzimidazoles with commercially available AD-mix- $\alpha$ [containing the (DHQ) 2 PHAL ligand]. ${ }^{1} \mathrm{H}$ NMR analysis of the corresponding Mosher esters revealed that the resulting diols were, in each case, virtually racemic. Presumably, the benzimidazole substrates can act as ligands for osmium and, thus, interfere with the, otherwise highly enantioselective, catalytic cycle.

\section{1-Allyl-2-(2-nitrophenyl)-1H-benzimidazole (51)}

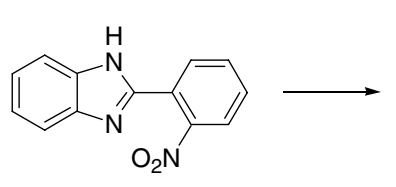

50

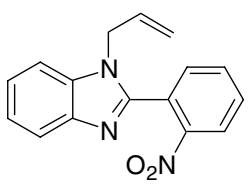

51

To an ice-cooled suspension of benzimidazole $\mathbf{5 0}^{6}(3.35 \mathrm{~g}, 14.0 \mathrm{mmol})$ in THF (40 mL) was added $\mathrm{NaH}(60 \% \mathrm{w} / \mathrm{w}, 616 \mathrm{mg}, 15.4 \mathrm{mmol})$ portionwise over $5 \mathrm{~min}$. After $10 \mathrm{~min}$ at $0{ }^{\circ} \mathrm{C}$, the resulting red solution was treated with allyl bromide $(1.6 \mathrm{~mL}, 18 \mathrm{mmol})$, and stirred at $0{ }^{\circ} \mathrm{C}$ $\rightarrow \mathrm{rt}$ for $16 \mathrm{~h}$. The reaction mixture was quenched with water, evaporated in vacuo, and partitioned between $\mathrm{CH}_{2} \mathrm{Cl}_{2}$ and water. The phases were separated, and the extraction was completed with additional portions of $\mathrm{CH}_{2} \mathrm{Cl}_{2}$. The combined organic extracts were dried $\left(\mathrm{MgSO}_{4}\right)$, and evaporated in vacuo to give a yellow oil. Purification by flash chromatography (silica gel, $\left.\mathrm{CH}_{2} \mathrm{Cl}_{2} \rightarrow \mathrm{CH}_{2} \mathrm{Cl}_{2} / \mathrm{EtOAc}, 2 / 1\right)$ gave the title compound $\mathbf{5 1}$ (3.80 g, 97\%) as a pale yellow solid: $\mathrm{R}_{f}=0.55\left(\mathrm{CH}_{2} \mathrm{Cl}_{2} /\right.$ EtOAc, $\left.3 / 1\right) ; \mathrm{mp} \mathrm{81.5-83.0}{ }^{\circ} \mathrm{C}$ (EtOAc/petroleum ether); ${ }^{1} \mathrm{H}$ NMR (400 MHz, CDCl $) \delta 4.64(\mathrm{~s}, 2 \mathrm{H}), 5.07(\sim \mathrm{d}, J=17.0 \mathrm{~Hz}, 1 \mathrm{H}), 5.21(\mathrm{~d}, J=10.5 \mathrm{~Hz}, 1 \mathrm{H})$, 5.84-5.94 (m, 1H), 7.28-7.47 (m, 3H), $7.62(\mathrm{~d}, J=7.0 \mathrm{~Hz}, 1 \mathrm{H}), 7.65-7.88(\mathrm{~m}, 3 \mathrm{H})$, and $8.19(\mathrm{~d}, J$ $=8.0 \mathrm{~Hz}, 1 \mathrm{H}) ;{ }^{13} \mathrm{C}\left\{{ }^{1} \mathrm{H}\right\} \mathrm{NMR}\left(63 \mathrm{MHz}, \mathrm{CDCl}_{3}\right) \delta 46.98,110.4,117.9,120.0,122.4,123.1$, 124.7, 125.9, 131.0, 131.6, 132.5, 133.1, 134.8, 143.0, 148.7, and 149.3; IR $\left(\mathrm{CHCl}_{3}\right) v_{\max } 1535$, 1459, 1401, and $1348 \mathrm{~cm}^{-1}$; MS (ESI) $\mathrm{m} / z$ (rel intensity) $280\left(100 \%, \mathrm{MH}^{+}\right)$; HRMS calcd for $\mathrm{C}_{16} \mathrm{H}_{14} \mathrm{~N}_{3} \mathrm{O}_{2}\left(\mathrm{MH}^{+}\right)$280.1086, found 280.1092; Anal. Calcd for $\mathrm{C}_{16} \mathrm{H}_{13} \mathrm{~N}_{3} \mathrm{O}_{2}$ : C, 68.81; H, 4.69; N, 15.05. Found: C, 68.74; H, 4.71; N, 15.13. 


\section{3-[2-(2-Nitrophenyl)benzimidazol-1-yl]propane-1,2-diol (20)}

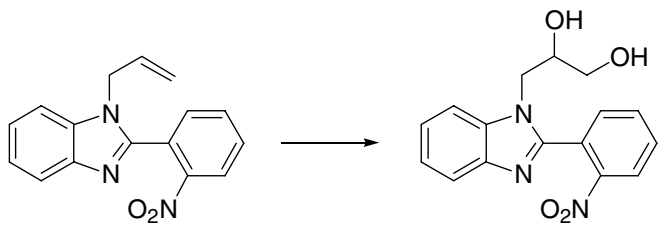

51

20

A solution of $\mathrm{AD}-\mathrm{mix}-\beta(6.2 \mathrm{~g})$ in ${ }^{t} \mathrm{BuOH}(25 \mathrm{~mL})$ and $\mathrm{H}_{2} \mathrm{O}(25 \mathrm{~mL})$ was stirred at $\mathrm{rt}$ for $1 \mathrm{~h}$. The mixture was cooled in an ice bath, and benzimidazole 51 (1.15 g, $4.12 \mathrm{mmol})$ was added. After $13 \mathrm{~h}$ at $0{ }^{\circ} \mathrm{C} \rightarrow \mathrm{rt}, \mathrm{Na}_{2} \mathrm{SO}_{3}(4.5 \mathrm{~g}, 36 \mathrm{mmol})$ was added, and the reaction mixture was stirred at $\mathrm{rt}$ for $1 \mathrm{~h}$. The resulting gray solution was diluted with $\mathrm{CH}_{2} \mathrm{Cl}_{2}$ and $\mathrm{H}_{2} \mathrm{O}$, and stirred for $5 \mathrm{~min}$. The phases were separated, and the extraction was completed with additional portions of $\mathrm{CH}_{2} \mathrm{Cl}_{2}$. The combined organic extracts were dried $\left(\mathrm{MgSO}_{4}\right)$, and evaporated in vacuo to give a yellow solid. Purification by flash chromatography (silica gel, EtOAc/Me ${ }_{2} \mathrm{CO}, 10 / 1$ ) gave the title compound 20 (950 mg, 74\%) as a yellow solid: $\mathrm{R}_{f}=0.15$ (EtOAc); mp 181.5-183.0 ${ }^{\circ} \mathrm{C}$ $\left(\right.$ EtOAc); $[\alpha]_{\mathrm{D}}=-0.5$ (c 0.61 in $\left.\mathrm{MeOH}\right) ;{ }^{1} \mathrm{H}$ NMR (400 MHz, $d_{6}$-DMSO) $\delta 3.27-3.40(\mathrm{~m}, 2 \mathrm{H})$, 3.84-3.90 (m, 1H), $3.96(\mathrm{dd}, J=14.5,8.5 \mathrm{~Hz}, 1 \mathrm{H}), 4.29(\mathrm{dd}, J=14.5,3.0 \mathrm{~Hz}, 1 \mathrm{H}), 4.76(\mathrm{t}, J=$ $5.5 \mathrm{~Hz}, 1 \mathrm{H}), 5.11(\mathrm{~d}, J=5.0 \mathrm{~Hz}, 1 \mathrm{H}), 7.26(\sim \mathrm{t}, J=7.0 \mathrm{~Hz}, 1 \mathrm{H}), 7.33(\mathrm{dt}, J=7.0,1.0 \mathrm{~Hz}, 1 \mathrm{H})$, $7.66(\mathrm{~d}, J=8.0 \mathrm{~Hz}, 1 \mathrm{H}), 7.71(\mathrm{~d}, J=8.0 \mathrm{~Hz}, 1 \mathrm{H}), 7.83(\mathrm{dt}, J=7.5,1.5 \mathrm{~Hz}, 1 \mathrm{H}), 7.90(\mathrm{dt}, J=7.5$, $1.0 \mathrm{~Hz}, 1 \mathrm{H}), 7.97(\mathrm{dd}, J=7.5,1.5 \mathrm{~Hz}, 1 \mathrm{H})$, and $8.21(\mathrm{dd}, J=8.0,1.0 \mathrm{~Hz}, 1 \mathrm{H}) ;{ }^{13} \mathrm{C}\left\{{ }^{1} \mathrm{H}\right\} \mathrm{NMR}$ (63 MHz, $d_{6}$-DMSO) $\delta 47.78,63.47,70.03,111.5,119.1,121.9,122.5,124.5,125.4,131.2$, 133.0, 133.3, 135.4, 142.6, 149.1, and 149.5; IR (KBr) $v_{\max } 3073,1523,1464,1446,1417$, and $1349 \mathrm{~cm}^{-1}$; MS (ESI) $\mathrm{m} / z$ (rel intensity) $314\left(100 \%, \mathrm{MH}^{+}\right)$; HRMS calcd for $\mathrm{C}_{16} \mathrm{H}_{16} \mathrm{~N}_{3} \mathrm{O}_{4}\left(\mathrm{MH}^{+}\right)$ 314.1141, found 314.1146; Anal. Calcd for $\mathrm{C}_{16} \mathrm{H}_{15} \mathrm{~N}_{3} \mathrm{O}_{4}$ : C, 61.34; H, 4.83; N, 13.41. Found: C, 61.46; H, 4.84; N, 13.50 .

$\left(2 R, 2^{\prime} R\right)-3,3,3-T^{\prime}$ rifluoro-2-methoxy-2-phenylpropionic acid 2-[2-(2-nitrophenyl)benzoimidazol-1-yl]-1-(3,3,3-trifluoro-2-methoxy-2-phenylpropionyloxymethyl)ethyl ester (52)

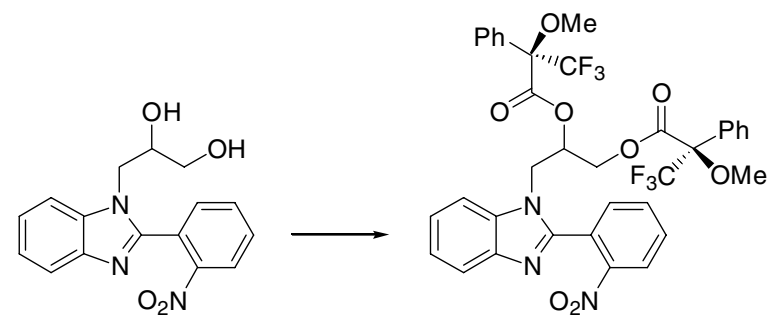

20

52 
To an ice-cooled solution of diol $20(20.0 \mathrm{mg}, 64 \mu \mathrm{mol})$ in $\mathrm{CH}_{2} \mathrm{Cl}_{2}(2 \mathrm{~mL})$ were added (R)-MTPA (59.8 mg, $0.26 \mathrm{mmol}$ ), DMAP (33 mg, $0.27 \mathrm{mmol}$ ), and DCC (158 mg, $0.77 \mathrm{mmol})$. The reaction mixture was stirred at $0{ }^{\circ} \mathrm{C} \rightarrow \mathrm{rt}$ for $40 \mathrm{~h}$, and the resulting white suspension filtered through a plug of cotton. The filtrate was diluted with EtOAc, and washed successively with satd $\mathrm{NaHCO}_{3}$, water, and brine. The organic layer was dried $\left(\mathrm{MgSO}_{4}\right)$, and evaporated in vacuo to give a pale yellow oil. Purification by flash chromatography (silica gel, $\mathrm{CH}_{2} \mathrm{Cl}_{2} \rightarrow$ $\mathrm{CH}_{2} \mathrm{Cl}_{2} /$ EtOAc, 10/1) gave the title compound $\mathbf{5 2}$ (44 mg, 92\%) as a pale yellow oil: $\mathrm{R}_{f}=0.25$ (petroleum ether/EtOAc, 2/1); ${ }^{1} \mathrm{H}$ NMR (400 MHz, $\left.\mathrm{CDCl}_{3}\right) \delta 2.92$ (s, 3H), $3.23(\mathrm{~s}, 6 \mathrm{H}), 3.29$ (s, $3 \mathrm{H}), 4.05-4.21(\mathrm{~m}, 6 \mathrm{H}), 4.57(\mathrm{dd}, J=13.0,3.0 \mathrm{~Hz}, 1 \mathrm{H}), 4.75(\mathrm{dd}, J=12.5,2.5 \mathrm{~Hz}, 1 \mathrm{H}), 5.49$ $5.59(\mathrm{~m}, 2 \mathrm{H}), 6.95-7.34(\mathrm{~m}, 28 \mathrm{H}), 7.50-7.77(\mathrm{~m}, 6 \mathrm{H})$, and 8.02-8.13 (m, 2H); IR $\left(\mathrm{CHCl}_{3}\right) v_{\max }$ 1758 and $1534 \mathrm{~cm}^{-1}$; MS (ESI) $\mathrm{m} / z$ (rel intensity) $768\left(90 \%, \mathrm{MNa}^{+}\right.$) and 746 (100); HRMS calcd for $\mathrm{C}_{36} \mathrm{H}_{29} \mathrm{~F}_{6} \mathrm{~N}_{3} \mathrm{NaO}_{8}\left(\mathrm{MNa}^{+}\right)$768.1757, found 768.1743.

\section{1-Allyl-2-(3-chloro-2-nitrophenyl)-4-methylbenzimidazole (53) and}

\section{1-allyl-2-(3-chloro-2-nitrophenyl)-7-methylbenzimidazole (54)}

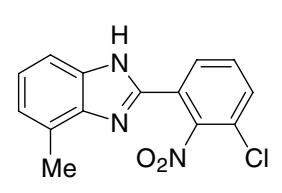

39

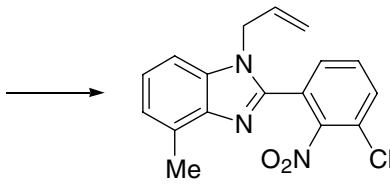

53

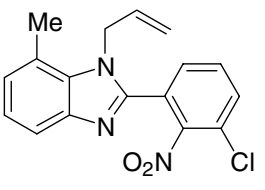

54

To a yellow solution of benzimidazole $39(3.00 \mathrm{~g}, 10.5 \mathrm{mmol})$ in THF $(25 \mathrm{~mL})$ was added $\mathrm{NaH}(60 \% \mathrm{w} / \mathrm{w}, 462 \mathrm{mg}, 11.6 \mathrm{mmol})$ portionwise at $0{ }^{\circ} \mathrm{C}$. After $15 \mathrm{~min}$ at $\mathrm{rt}$, the resulting deep-orange solution was treated with allyl bromide $(1.2 \mathrm{~mL}, 13.6 \mathrm{mmol})$, and stirred at $\mathrm{rt}$ for 18 h. The reaction mixture was quenched with water, evaporated in vacuo, and partitioned between $\mathrm{CH}_{2} \mathrm{Cl}_{2}$ and water. The phases were separated, and the extraction was completed with additional portions of $\mathrm{CH}_{2} \mathrm{Cl}_{2}$. The combined organic extracts were dried $\left(\mathrm{MgSO}_{4}\right)$, and evaporated in vacuo to give a yellow solid. Repetitive purification by flash chromatography (silica gel, $\mathrm{CH}_{2} \mathrm{Cl}_{2}$ $\rightarrow \mathrm{CH}_{2} \mathrm{Cl}_{2}$ /EtOAc, 20/1) gave the title compound $\mathbf{5 3}(2.39 \mathrm{~g}, 70 \%)$ as a pale yellow solid, and the title compound $54(618 \mathrm{mg}, 18 \%)$ as a yellow solid. ${ }^{7}$ Benzimidazole 53: $\mathbf{R}_{f}=0.55$ (EtOAc/petroleum ether, 1/1); mp 124.5-125.5 ${ }^{\circ} \mathrm{C}$ (EtOAc/petroleum ether); ${ }^{1} \mathrm{H}$ NMR (400 $\left.\mathrm{MHz} \mathrm{CDCl}_{3}\right) \delta 2.68(\mathrm{~s}, 3 \mathrm{H}), 4.72-4.73(\mathrm{~m}, 2 \mathrm{H}), 5.05(\mathrm{~d}, J=17.0 \mathrm{~Hz}, 1 \mathrm{H}), 5.27(\mathrm{~d}, J=10.0 \mathrm{~Hz}$,

\footnotetext{
${ }^{7}$ As the product distribution in $N$-alkylations of unsymmetrical 2-aryl- $1 H$-benzimidazoles (e.g., 39) is usually strongly biased towards a sterically less hindered of the two possible isomers, the major product $\mathbf{5 3}$ was assigned the structure as depicted, with the allyl substituent attached to the sterically more accessible benzimidazole nitrogen.
} 
1H), $5.95(\mathrm{ddt}, J=17.0,10.0,3.5 \mathrm{~Hz}, 1 \mathrm{H}), 7.13(\mathrm{~d}, J=6.0 \mathrm{~Hz}, 1 \mathrm{H}), 7.21-7.28(\mathrm{~m}, 2 \mathrm{H}), 7.52-$ $7.58(\mathrm{~m}, 2 \mathrm{H})$, and $7.67(\sim \mathrm{d}, J=7.5 \mathrm{~Hz}, 1 \mathrm{H}) ;{ }^{13} \mathrm{C}\left\{{ }^{1} \mathrm{H}\right\}$ NMR $\left(101 \mathrm{MHz}, \mathrm{CDCl}_{3}\right) \delta 16.51,47.10$, 108.0, 117.8, 123.2, 123.7, 125.9, 126.5, 129.4, 130.7 ( $2 \times \mathrm{C}), 131.8,132.1,134.7,142.4,145.8$, and 149.5; IR $\left(\mathrm{CHCl}_{3}\right) v_{\max } 1546,1455$, and $1364 \mathrm{~cm}^{-1}$; MS (ESI) $\mathrm{m} / \mathrm{z}$ (rel intensity) 350 (100\%, $\mathrm{MNa}^{+}$) and 328 (50); HRMS calcd for $\mathrm{C}_{17} \mathrm{H}_{14} \mathrm{ClN}_{3} \mathrm{NaO}_{2}\left(\mathrm{MNa}^{+}\right)$350.0672, found 350.0670; Anal. Calcd for $\mathrm{C}_{13} \mathrm{H}_{14} \mathrm{ClN}_{3} \mathrm{O}_{2}: \mathrm{C}, 62.30 ; \mathrm{H}, 4.31 ; \mathrm{Cl}, 10.82 ; \mathrm{N}, 12.82$. Found: $\mathrm{C}, 62.46 ; \mathrm{H}, 4.29$; $\mathrm{Cl}, 10.99 ; \mathrm{N}, 12.86$. Benzimidazole 54: $\mathrm{R}_{f}=0.50$ (EtOAc/petroleum ether, 1/1); mp 144.0-145.0 ${ }^{\circ} \mathrm{C}$ (EtOAc/petroleum ether); ${ }^{1} \mathrm{H}$ NMR $\left(400 \mathrm{MHz}, \mathrm{CDCl}_{3}\right) \delta 2.64$ (s, 3H), 4.75 ( dd, $J=17.0,2.0$ $\mathrm{Hz}, 1 \mathrm{H}), 4.84-4.86(\mathrm{~m}, 2 \mathrm{H}), 5.22(\sim \mathrm{dd}, J=10.5,1.5 \mathrm{~Hz}, 1 \mathrm{H}), 5.98$ (ddt, $J=17.0,10.5,4.0 \mathrm{~Hz}$, $1 \mathrm{H}), 7.03(\mathrm{~d}, J=7.5 \mathrm{~Hz}, 1 \mathrm{H}), 7.16(\mathrm{t}, J=7.5 \mathrm{~Hz}, 1 \mathrm{H}), 7.47-7.52(\mathrm{~m}, 2 \mathrm{H})$, and 7.60-7.66 (m, 2H); ${ }^{13} \mathrm{C}\left\{{ }^{1} \mathrm{H}\right\}$ NMR $\left(101 \mathrm{MHz}, \mathrm{CDCl}_{3}\right) \delta 17.96,47.82,116.7,118.6,121.5,122.9,125.9,126.3$, 129.3, 130.7, 132.2, 133.7, 134.2, 143.4, 147.4, and 149.5 (one $\mathrm{C}$ atom obscured); $\mathrm{R}\left(\mathrm{CHCl}_{3}\right)$ $v_{\max } 1545,1454,1394$, and $1363 \mathrm{~cm}^{-1}$; MS (ESI) $\mathrm{m} / z$ (rel intensity) $350\left(100 \%, \mathrm{MNa}^{+}\right)$and 328 (65); HRMS calcd for $\mathrm{C}_{17} \mathrm{H}_{15} \mathrm{ClN}_{3} \mathrm{O}_{2}\left(\mathrm{MH}^{+}\right)$328.0853, found 328.0859; Anal. Calcd for $\mathrm{C}_{17} \mathrm{H}_{14} \mathrm{ClN}_{3} \mathrm{O}_{2}$ : C, 62.30; H, 4.31; Cl, 10.82; N, 12.82. Found: C, 62.52; H, 4.30; Cl, 10.89; N, 12.90 .

\section{3-[2-(3-Chloro-2-nitrophenyl)-4-methyl-benzimidazol-1-yl]propane-1,2-diol (24)}



A solution of AD-mix $\beta(5.5 \mathrm{~g})$ in ${ }^{t} \mathrm{BuOH}(24 \mathrm{~mL})$ and $\mathrm{H}_{2} \mathrm{O}(24 \mathrm{~mL})$ was stirred at $\mathrm{rt}$ for $45 \mathrm{~min}$. The mixture was cooled to $0{ }^{\circ} \mathrm{C}$, and benzimidazole $53(1.07 \mathrm{~g}, 3.28 \mathrm{mmol})$ was added. After $70 \mathrm{~h}$ at $0{ }^{\circ} \mathrm{C} \rightarrow \mathrm{rt}, \mathrm{Na}_{2} \mathrm{SO}_{3}(4.0 \mathrm{~g}, 32 \mathrm{mmol})$ was added, and the reaction mixture was stirred at $\mathrm{rt}$ for $1 \mathrm{~h}$. The resulting gray suspension was diluted with $\mathrm{CH}_{2} \mathrm{Cl}_{2}$ and $\mathrm{H}_{2} \mathrm{O}$, and stirred for $5 \mathrm{~min}$. The phases were separated, and the extraction was completed with additional portions of $\mathrm{CH}_{2} \mathrm{Cl}_{2}$. The combined organic extracts were dried $\left(\mathrm{MgSO}_{4}\right)$, and evaporated in vacuo to give a white foam. Purification by flash chromatography (silica gel, $\mathrm{CH}_{2} \mathrm{Cl}_{2} \rightarrow$ EtOAc) gave the recovered starting material $53(116 \mathrm{mg}, 11 \%)$ and the title compound $24(1.01 \mathrm{~g}, 85 \%)$ as a yellow solid: $\mathrm{R}_{f}=0.50$ (EtOAc); mp 165.5-166.5 ${ }^{\circ} \mathrm{C}$ (EtOAc/petroleum ether); ${ }^{1} \mathrm{H}$ NMR (400 
MHz, $d_{6}$-DMSO) $\delta 2.50(\mathrm{~s}, 3 \mathrm{H}), 3.29-3.41(\mathrm{~m}, 2 \mathrm{H}), 3.82-3.94(\mathrm{~m}, 1 \mathrm{H}), 4.04(\mathrm{dd}, J=14.5,9.0$ $\mathrm{Hz}, 1 \mathrm{H}), 4.35$ (dd, $J=14.5,3.0 \mathrm{~Hz}, 1 \mathrm{H}), 4.76(\mathrm{t}, J=5.5 \mathrm{~Hz}, 1 \mathrm{H}), 5.14(\mathrm{~d}, J=5.0 \mathrm{~Hz}, 1 \mathrm{H}), 7.08$ $(\mathrm{d}, J=7.5 \mathrm{~Hz}, 1 \mathrm{H}), 7.23(\mathrm{t}, J=7.5 \mathrm{~Hz}, 1 \mathrm{H}), 7.50(\mathrm{~d}, J=8.0 \mathrm{~Hz}, 1 \mathrm{H}), 7.82(\mathrm{t}, J=8.0 \mathrm{~Hz}, 1 \mathrm{H})$, $7.98(\mathrm{~d}, J=8.0 \mathrm{~Hz}, 1 \mathrm{H})$, and $8.11(\mathrm{~d}, J=7.5 \mathrm{~Hz}, 1 \mathrm{H}) ;{ }^{13} \mathrm{C}\left\{{ }^{1} \mathrm{H}\right\} \mathrm{NMR}\left(101 \mathrm{MHz}, d_{6}\right.$-DMSO) $\delta$ $16.12,47.85,63.47,69.82,109.1,122.4,123.0,124.6,125.5,129.0,131.0,132.0(2 \times \mathrm{C}), 135.0$, 141.8, 146.2, and 148.7; IR (KBr) $v_{\max } 1539,1459,1439$, and $1364 \mathrm{~cm}^{-1}$; MS (ESI) $\mathrm{m} / z$ (rel intensity) $384\left(85 \%, \mathrm{MNa}^{+}\right)$and 362 (100); HRMS calcd for $\mathrm{C}_{17} \mathrm{H}_{16} \mathrm{ClN}_{3} \mathrm{NaO}_{4}\left(\mathrm{MNa}^{+}\right)$384.0727, found 384.0744; Anal. Calcd for $\mathrm{C}_{17} \mathrm{H}_{16} \mathrm{ClN}_{3} \mathrm{O}_{4}$ : C, 56.44; H, 4.46; Cl, 9.80; N, 11.61. Found: C, $56.58 ; \mathrm{H}, 4.41 ; \mathrm{Cl}, 9.79 ; \mathrm{N}, 11.65$.

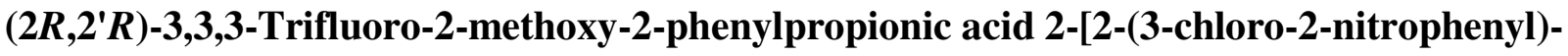

\section{4-methylbenzoimidazol-1-yl]-1-(3,3,3-trifluoro-2-methoxy-2-phenylpropionyloxymethyl)- ethyl ester (55)}

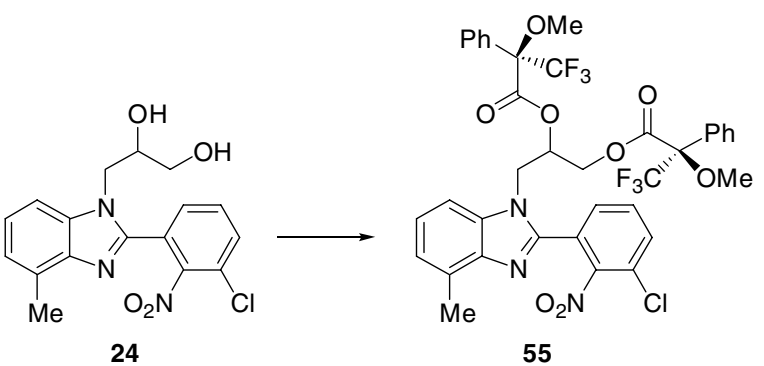

To an ice-cooled solution of diol $24(15.0 \mathrm{mg}, 42 \mu \mathrm{mol})$ in $\mathrm{CH}_{2} \mathrm{Cl}_{2}(3 \mathrm{~mL})$ were added (R)-MTPA (69 mg, $0.30 \mathrm{mmol})$, DMAP (38 mg, $0.31 \mathrm{mmol})$, and DCC (182 mg, $0.88 \mathrm{mmol})$. The reaction mixture was stirred at $0{ }^{\circ} \mathrm{C} \rightarrow \mathrm{rt}$ for $48 \mathrm{~h}$, and the resulting white suspension filtered through a plug of cotton. The filtrate was diluted with EtOAc, and washed successively with satd $\mathrm{NaHCO}_{3}$, water, and brine. The organic layer was dried $\left(\mathrm{MgSO}_{4}\right)$, and evaporated in vacuo to give a pale yellow oil. Purification by flash chromatography (silica gel, $\mathrm{CH}_{2} \mathrm{Cl}_{2} \rightarrow$ $\mathrm{CH}_{2} \mathrm{Cl}_{2} / \mathrm{EtOAc}$, 10/1) gave the title compound $55(29 \mathrm{mg}, 88 \%)$ as a clear oil: $\mathrm{R}_{f}=0.25$ (petroleum ether/EtOAc, 3/1); ${ }^{1} \mathrm{H}$ NMR (400 MHz, $\left.\mathrm{CDCl}_{3}\right) \delta 2.55$ (s, 3H), 2.57 (s, 3H), 2.91 (s, $3 \mathrm{H}), 3.28(\mathrm{~s}, 6 \mathrm{H}), 3.31(\mathrm{~s}, 3 \mathrm{H}), 4.08-4.27(\mathrm{~m}, 6 \mathrm{H}), 4.57(\mathrm{dd}, J=13.0,3.0 \mathrm{~Hz}, 1 \mathrm{H}), 4.75(\mathrm{dd}, J=$ 13.0, 3.0 Hz, 1H), 5.56-5.62 (m, 2H), 7.02-7.39 (m, 30H), and 7.52-7.59 (m, 2H); IR $\left(\mathrm{CHCl}_{3}\right)$ $v_{\max } 1759,1545,1453,1269$, and $1231 \mathrm{~cm}^{-1}$; MS (ESI) $\mathrm{m} / z$ (rel intensity) $816\left(90 \%, \mathrm{MNa}^{+}\right)$and 794 (100); HRMS calcd for $\mathrm{C}_{37} \mathrm{H}_{30} \mathrm{ClF}_{6} \mathrm{~N}_{3} \mathrm{NaO}_{8}\left(\mathrm{MNa}^{+}\right)$816.1523, found 816.1529. 


\section{1-Allyl-2-(3-chloro-2-nitrophenyl)-1H-benzimidazole (56)}

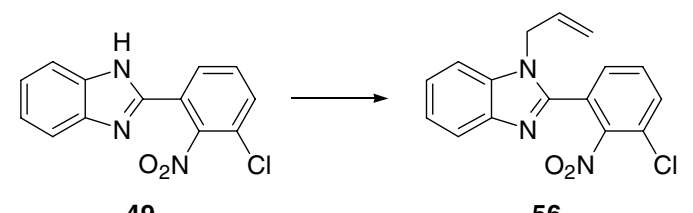

49

56

To a suspension of benzimidazole $49(2.00 \mathrm{~g}, 7.3 \mathrm{mmol})$ in THF $(25 \mathrm{~mL})$ was added $\mathrm{NaH}$ $\left(60 \% \mathrm{w} / \mathrm{w}, 321 \mathrm{mg}, 8.0 \mathrm{mmol}\right.$ ) portionwise at $0{ }^{\circ} \mathrm{C}$. After $15 \mathrm{~min}$ at $\mathrm{rt}$, the resulting red-brown solution was treated with allyl bromide $(821 \mu \mathrm{L}, 8 \mathrm{mmol})$, and stirred at $\mathrm{rt}$ for $20 \mathrm{~h}$. The reaction mixture was quenched with water, evaporated in vacuo, and partitioned between $\mathrm{CH}_{2} \mathrm{Cl}_{2}$ and water. The phases were separated, and the extraction was completed with additional portions of $\mathrm{CH}_{2} \mathrm{Cl}_{2}$. The combined organic extracts were dried $\left(\mathrm{MgSO}_{4}\right)$, and evaporated in vacuo to give a yellow solid. Purification by flash chromatography (silica gel, $\mathrm{CH}_{2} \mathrm{Cl}_{2} \rightarrow \mathrm{CH}_{2} \mathrm{Cl}_{2} / \mathrm{EtOAc}$, 20/1) gave the title compound $\mathbf{5 6}(2.24 \mathrm{~g}, 98 \%)$ as a white solid: $\mathbf{R}_{f}=0.45$ (EtOAc/petroleum ether, 1/1); mp 148.5-149.5 ${ }^{\circ} \mathrm{C}$ (EtOAc/petroleum ether); ${ }^{1} \mathrm{H}$ NMR (400 MHz, $\left.\mathrm{CDCl}_{3}\right) \delta$ 4.69-4.71 (m, 2H), $5.01(\mathrm{~d}, J=17.0 \mathrm{~Hz}, 1 \mathrm{H}), 5.23(\mathrm{~d}, J=10.5 \mathrm{~Hz}, 1 \mathrm{H}), 5.92(\mathrm{ddt}, J=17.0,10.5,5.0 \mathrm{~Hz}, 1 \mathrm{H})$, 7.24-7.37 (m, 3H), 7.48 (dd, $J=7.5,1.5 \mathrm{~Hz}, 1 \mathrm{H}), 7.52(\mathrm{t}, J=7.5 \mathrm{~Hz}, 1 \mathrm{H}), 7.64(\mathrm{dd}, J=8.0,1.5$ $\mathrm{Hz}, 1 \mathrm{H})$, and 7.75-7.79 $(\mathrm{m}, 1 \mathrm{H}) ;{ }^{13} \mathrm{C}\left\{{ }^{1} \mathrm{H}\right\} \mathrm{NMR}\left(101 \mathrm{MHz}, \mathrm{CDCl}_{3}\right) \delta 47.04,110.6,117.9,120.6$, $122.9,123.7,125.6,126.5,129.2,130.8,131.7,132.2,135.1,142.9,146.7$, and 149.4; IR $\left(\mathrm{CHCl}_{3}\right) v_{\max } 1546,1458,1441,1393$, and $1363 \mathrm{~cm}^{-1}$; MS (ESI) $\mathrm{m} / \mathrm{z}$ (rel intensity) 314 (100\%, $\mathrm{MH}^{+}$); HRMS calcd for $\mathrm{C}_{16} \mathrm{H}_{13} \mathrm{ClN}_{3} \mathrm{O}_{2}\left(\mathrm{MH}^{+}\right)$314.0696, found 314.0709; Anal. Calcd for $\mathrm{C}_{16} \mathrm{H}_{12} \mathrm{ClN}_{3} \mathrm{O}_{2}: \mathrm{C}, 61.25 ; \mathrm{H}, 3.86 ; \mathrm{Cl}, 11.30 ; \mathrm{N}, 13.39$. Found: $\mathrm{C}, 61.31 ; \mathrm{H}, 3.89 ; \mathrm{Cl}, 11.38 ; \mathrm{N}$, 13.35 .

\section{3-[2-(3-Chloro-2-nitrophenyl)benzimidazol-1-yl]propane-1,2-diol (57)}

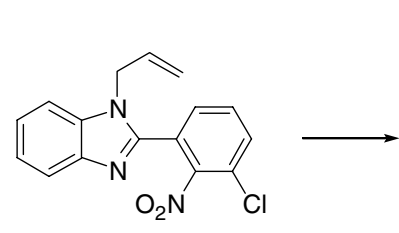

56

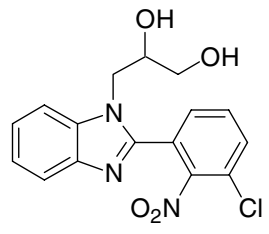

57

A solution of AD-mix $-\beta(7.2 \mathrm{~g})$ in ${ }^{t} \mathrm{BuOH}(29 \mathrm{~mL})$ and $\mathrm{H}_{2} \mathrm{O}(29 \mathrm{~mL})$ was stirred at $\mathrm{rt}$ for $1 \mathrm{~h}$. The reaction mixture was cooled in an ice bath, and benzimidazole $56(1.50 \mathrm{~g}, 4.78 \mathrm{mmol})$ was added. After $45 \mathrm{~h}$ at $0{ }^{\circ} \mathrm{C} \rightarrow \mathrm{rt}, \mathrm{Na}_{2} \mathrm{SO}_{3}(5.0 \mathrm{~g}, 40 \mathrm{mmol})$ was added, and the reaction mixture was stirred at $\mathrm{rt}$ for $1 \mathrm{~h}$. The resulting gray suspension was diluted with $\mathrm{CH}_{2} \mathrm{Cl}_{2}$ and 
$\mathrm{H}_{2} \mathrm{O}$, and stirred for $5 \mathrm{~min}$. The phases were separated, and the extraction was completed with additional portions of $\mathrm{CH}_{2} \mathrm{Cl}_{2}$. The combined organic extracts were dried $\left(\mathrm{MgSO}_{4}\right)$, and evaporated in vacuo to give an off-white solid. Purification by flash chromatography (silica gel, EtOAc $\rightarrow$ EtOAc/MeOH, 50/1) gave the recovered starting material 56 (869 mg, 58\%) and the title compound 57 (527 mg, 32\%). Diol 57: a white solid: $\mathrm{R}_{f}=0.20$ (EtOAc); mp 187.5-189.0 ${ }^{\circ} \mathrm{C}$ $\left(\right.$ EtOAc); $[\alpha]_{\mathrm{D}}=+0.8(c 0.62$ in $\mathrm{MeOH}) ;{ }^{1} \mathrm{H}$ NMR (400 MHz, $d_{6}$-DMSO) $\delta 3.29-3.42(\mathrm{~m}, 2 \mathrm{H})$, 3.86-3.95 (m, 1H), 4.09 (dd, $J=14.5,9.0 \mathrm{~Hz}, 1 \mathrm{H}), 4.39$ (dd, $J=14.5,3.0 \mathrm{~Hz}, 1 \mathrm{H}), 4.79$ (t, $J=$ $5.5 \mathrm{~Hz}, 1 \mathrm{H}), 5.18(\mathrm{~d}, J=5.0 \mathrm{~Hz}, 1 \mathrm{H}), 7.27(\mathrm{t}, J=8.0 \mathrm{~Hz}, 1 \mathrm{H}), 7.34(\mathrm{t}, J=7.0 \mathrm{~Hz}, 1 \mathrm{H}), 7.68(\mathrm{~d}, J$ $=8.5 \mathrm{~Hz}, 1 \mathrm{H}), 7.70(\mathrm{~d}, J=9.0 \mathrm{~Hz}, 1 \mathrm{H}), 7.82(\mathrm{t}, J=8.0 \mathrm{~Hz}, 1 \mathrm{H}), 7.97(\mathrm{~d}, J=8.0 \mathrm{~Hz}, 1 \mathrm{H})$, and $8.14(\mathrm{~d}, J=8.0 \mathrm{~Hz}, 1 \mathrm{H}) ;{ }^{13} \mathrm{C}\left\{{ }^{1} \mathrm{H}\right\} \mathrm{NMR}\left(101 \mathrm{MHz}, d_{6}\right.$-DMSO) $\delta 47.78,63.49,69.88,111.7$, 119.5, 122.3, 123.1, 124.7, 125.2, 131.0, 132.0, 132.1, 135.5, 142.4, 147.0, and 148.7; IR (KBr) $v_{\max } 3061,1528,1462,1401$, and $1358 \mathrm{~cm}^{-1}$; MS (ESI) $\mathrm{m} / z$ (rel intensity) $348\left(100 \%, \mathrm{MH}^{+}\right)$; HRMS calcd for $\mathrm{C}_{16} \mathrm{H}_{15} \mathrm{ClN}_{3} \mathrm{O}_{4}\left(\mathrm{MH}^{+}\right)$348.0751, found 348.0764; Anal. Calcd for $\mathrm{C}_{16} \mathrm{H}_{14} \mathrm{ClN}_{3} \mathrm{O}_{4}$ : C, 55.26; H, 4.06; Cl, 10.19; N, 12.08. Found: C, 55.28; H, 4.09; Cl, 10.44; N, 12.01 .

\subsection{Intramolecular $S_{\mathrm{N}} A \mathbf{r}$ Reactions with Monoalcohols}

\section{(S)-(+)-6-tert-Butyl-11-[1,3]dioxolan-2-yl-6,7-dihydro-5-oxa-7a,12-diazadibenzo[a,e]azulene}

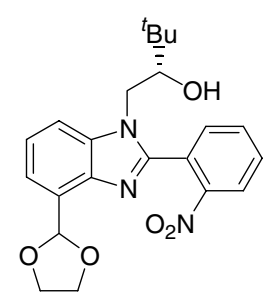

$(+)-1$



$(+)-3$

Method 1 (attempted etherification with 1,8-dibromooctane):

To a solution of alcohol (+)-1 ${ }^{8}(98 \%$ ee, $188 \mathrm{mg}, 0.46 \mathrm{mmol})$ in anhydrous DMF (2 mL) was added $\mathrm{NaH}(60 \% \mathrm{w} / \mathrm{w}, 20 \mathrm{mg}, 0.5 \mathrm{mmol})$, and the reaction mixture was stirred at $\mathrm{rt}$ for 50 min. 1,8-dibromooctane ( $42 \mu \mathrm{L}, 0.23 \mathrm{mmol}$ ) was added, and the mixture was stirred at $\mathrm{rt}$ for 21 h. The reaction mixture was quenched with water, and diluted with EtOAc. The organic phase

\footnotetext{
${ }^{8}$ Alcohols $\mathbf{1}$ and $\mathbf{1 2}$ were prepared as part of our studies on the synthesis of optically pure, strapped cyclic bis(benzimidazole) ligands. Results of these studies will be reported in due course.
} 
was washed repeatedly with water, dried $\left(\mathrm{MgSO}_{4}\right)$, and evaporated in vacuo to give an off-white solid. Purification by flash chromatography (silica gel, $\mathrm{CH}_{2} \mathrm{Cl}_{2} \rightarrow \mathrm{CH}_{2} \mathrm{Cl}_{2} / \mathrm{EtOAc}, 10 / 1$ ) gave the title compound (+)-3 (159 mg, 95\%) as a white solid. CSP HPLC analysis (Figure S1) revealed it to be of $98 \%$ optical purity.

\section{Method 2:}

To a solution of alcohol (+)-1 $\mathbf{1}^{8}(98 \%$ ee, $337 \mathrm{mg}, 0.82 \mathrm{mmol})$ in anhydrous DMF (3 mL) was added $\mathrm{NaH}(60 \% \mathrm{w} / \mathrm{w}, 36 \mathrm{mg}, 0.9 \mathrm{mmol})$. After $15 \mathrm{~h}$ at $\mathrm{rt}$, the reaction mixture was quenched with water, and diluted with EtOAc. The organic phase was washed repeatedly with water, dried $\left(\mathrm{MgSO}_{4}\right)$, and evaporated in vacuo to give an off-white solid. Purification by flash chromatography (silica gel, $\mathrm{CH}_{2} \mathrm{Cl}_{2} \rightarrow \mathrm{CH}_{2} \mathrm{Cl}_{2} / \mathrm{EtOAc}, 10 / 1$ ) gave the title compound (+)-3 (246 mg, 83\%) as a white solid: $\mathrm{mp} 150.0-152.0{ }^{\circ} \mathrm{C}\left(\mathrm{EtOAc} /\right.$ petroleum ether); $[\alpha]_{\mathrm{D}}=+147(c 0.90$ in $\left.\mathrm{CHCl}_{3}\right)$.

\section{6,11-Dimethyl-6,7-dihydro-5-oxa-7a,12-diaza-dibenzo[a,e $]$ azulene (5)}

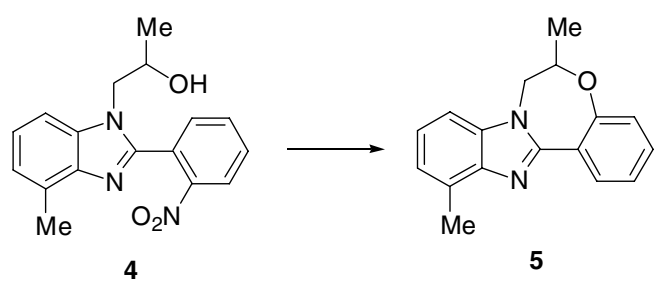

To a solution of alcohol 4 (142 mg, $0.46 \mathrm{mmol})$ in anhydrous DMF (1 mL) was added $\mathrm{NaH}(60 \% \mathrm{w} / \mathrm{w}, 20 \mathrm{mg}, 0.50 \mathrm{mmol})$. After $14 \mathrm{~h}$ at $\mathrm{rt}$, the reaction mixture was quenched with water, and diluted with EtOAc. The organic phase was washed repeatedly with water, dried $\left(\mathrm{MgSO}_{4}\right)$, and evaporated in vacuo to give an off-white solid. Purification by flash chromatography (silica gel, $\mathrm{CH}_{2} \mathrm{Cl}_{2} \rightarrow \mathrm{CH}_{2} \mathrm{Cl}_{2} / \mathrm{EtOAc}, 10 / 1$ ) gave the title compound 5 (94 $\mathrm{mg}$, $78 \%$ ) as a white solid: $\mathrm{R}_{f}=0.35$ (petroleum ether/EtOAc, 9/1); ${ }^{1} \mathrm{H}$ NMR $\left(250 \mathrm{MHz}, \mathrm{CDCl}_{3}\right) \delta$ $1.44(\mathrm{~d}, J=6.5 \mathrm{~Hz}, 3 \mathrm{H}), 2.65(\mathrm{~s}, 3 \mathrm{H}), 4.05(\mathrm{dd}, J=14.0,8.5 \mathrm{~Hz}, 1 \mathrm{H}), 4.22(\mathrm{dd}, J=14.0,2.0 \mathrm{~Hz}$, $1 \mathrm{H}), 4.43(\mathrm{ddq}, J=8.5,6.5,2.0 \mathrm{~Hz}, 1 \mathrm{H}), 6.94-7.14(\mathrm{~m}, 5 \mathrm{H}), 7.24(\mathrm{ddd}, J=8.0,7.5,2.0 \mathrm{~Hz}, 1 \mathrm{H})$, and $8.59(\mathrm{dd}, J=8.0,2.0 \mathrm{~Hz}, 1 \mathrm{H}) ;{ }^{13} \mathrm{C}\left\{{ }^{1} \mathrm{H}\right\} \mathrm{NMR}\left(101 \mathrm{MHz}, \mathrm{CDCl}_{3}\right) \delta 19.07,21.80,53.85$, $78.41,108.8,122.4,123.6,125.0,125.3,125.6,132.3,133.5,133.7,138.3,144.6,151.9$, and 158.1; IR $\left(\mathrm{CHCl}_{3}\right) v_{\max } 1607,1576,1473,1449,1385$, and $1233 \mathrm{~cm}^{-1}$; MS (ESI) m/z (rel intensity) $265\left(100 \%, \mathrm{MH}^{+}\right)$; HRMS calcd for $\mathrm{C}_{17} \mathrm{H}_{16} \mathrm{~N}_{2} \mathrm{NaO}\left(\mathrm{MNa}^{+}\right)$287.1160, found 287.1186. 


\section{6-Butyl-11-methyl-6,7-dihydro-5-oxa-7a,12-diazadibenzo[a,e]azulene (7)}

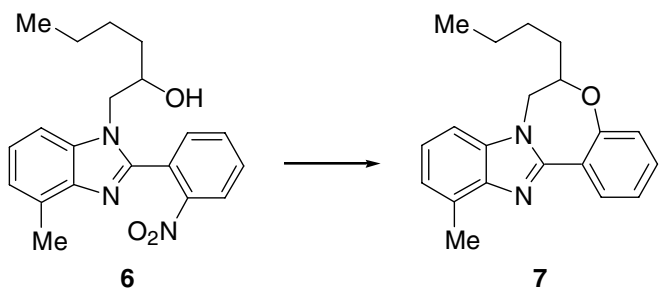

To a solution of alcohol 6 (664 $\mathrm{mg}, 1.88 \mathrm{mmol})$ in anhydrous DMF (62 mL) was added $\mathrm{NaH}(60 \% \mathrm{w} / \mathrm{w}, 83 \mathrm{mg}, 2.1 \mathrm{mmol})$ to give a black solution. After $17 \mathrm{~h}$ at $\mathrm{rt}$, the reaction mixture was quenched with water, and diluted with EtOAc. The organic phase was washed repeatedly with water, dried $\left(\mathrm{MgSO}_{4}\right)$, and evaporated in vacuo to give an off-white solid. Purification by flash chromatography (silica gel, $\mathrm{CH}_{2} \mathrm{Cl}_{2} \rightarrow \mathrm{CH}_{2} \mathrm{Cl}_{2} / \mathrm{EtOAc}, 10 / 1$ ) gave the title compound 7 (432 mg, 75\%) as a white solid: $\mathrm{R}_{f}=0.45$ (petroleum ether/EtOAc, 9/1); ${ }^{1} \mathrm{H}$ NMR $(250 \mathrm{MHz}$, $\left.\mathrm{CDCl}_{3}\right) \delta 0.88(\mathrm{t}, J=7.0 \mathrm{~Hz}, 3 \mathrm{H}), 1.20-1.91(\mathrm{~m}, 6 \mathrm{H}), 2.67(\mathrm{~s}, 3 \mathrm{H}), 4.03(\mathrm{dd}, J=14.0,9.0 \mathrm{~Hz}$, $1 \mathrm{H}), 4.22(\mathrm{dd}, J=14.0,2.0 \mathrm{~Hz}, 1 \mathrm{H}), 6.95-7.14(\mathrm{~m}, 5 \mathrm{H}), 7.24(\mathrm{ddd}, J=8.0,7.0,2.0 \mathrm{~Hz}, 1 \mathrm{H})$, and $8.63(\mathrm{dd}, J=8.0,2.0 \mathrm{~Hz}, 1 \mathrm{H}) ;{ }^{13} \mathrm{C}\left\{{ }^{1} \mathrm{H}\right\} \mathrm{NMR}\left(63 \mathrm{MHz}, \mathrm{CDCl}_{3}\right) \delta 14.47,17.15,22.92,28.17$, 33.50, 51.21, 80.16, 107.0, 120.7, 121.4, 123.0, 123.3, 123.6, 130.3, 131.5, 131.8, 136.5, 142.7, 149.8, and 156.6; IR $\left(\mathrm{CHCl}_{3}\right) v_{\max } 1605,1576,1427,1447,1376$, and $1321 \mathrm{~cm}^{-1}$; MS (ESI) $\mathrm{m} / z$ (rel intensity) 307 (100\%, $\mathrm{MH}^{+}$); HRMS calcd for $\mathrm{C}_{20} \mathrm{H}_{23} \mathrm{~N}_{2} \mathrm{O}\left(\mathrm{MH}^{+}\right)$307.1810, found 307.1798.

\section{6-tert-Butyl-6,7-dihydro-5-oxa-7a,12-diazadibenzo[a,e $]$ azulene (9)}

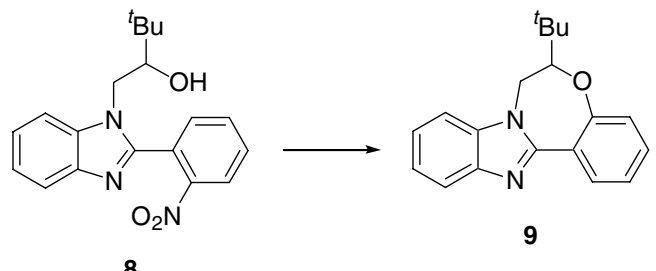

To a solution of alcohol 8 (100 mg, $0.29 \mathrm{mmol})$ in anhydrous DMF (2 mL) was added $\mathrm{NaH}(60 \% \mathrm{w} / \mathrm{w}, 12.8 \mathrm{mg}, 0.32 \mathrm{mmol})$ to give a dark-green solution. After $11 \mathrm{~h}$ at $\mathrm{rt}$, the reaction mixture was quenched with water, and diluted with EtOAc. The organic phase was washed repeatedly with water, dried $\left(\mathrm{MgSO}_{4}\right)$, and evaporated in vacuo to give a yellow solid. Purification by flash chromatography (silica gel, $\mathrm{CH}_{2} \mathrm{Cl}_{2} \rightarrow \mathrm{CH}_{2} \mathrm{Cl}_{2} / \mathrm{EtOAc}$, 10/1) gave the title compound 9 (74 mg, 86\%) as a white solid: $\mathrm{R}_{f}=0.65\left(\mathrm{CH}_{2} \mathrm{Cl}_{2} / \mathrm{EtOAc}, 9 / 1\right) ; \mathrm{mp} 149.0-150.0{ }^{\circ} \mathrm{C}$ (EtOAc/petroleum ether); ${ }^{1} \mathrm{H}$ NMR (400 MHz, $\left.\mathrm{CDCl}_{3}\right) \delta 1.09(\mathrm{~s}, 9 \mathrm{H}), 3.83(\mathrm{dd}, J=9.5,1.0 \mathrm{~Hz}$, $1 \mathrm{H}), 4.19(\mathrm{dd}, J=13.5,9.5 \mathrm{~Hz}, 1 \mathrm{H}), 4.49(\mathrm{dd}, J=13.5,1.0 \mathrm{~Hz}, 1 \mathrm{H}), 7.01(\sim \mathrm{d}, J=8.0 \mathrm{~Hz}, 1 \mathrm{H})$, 
$7.09(\mathrm{dt}, J=8.0,0.5 \mathrm{~Hz}, 1 \mathrm{H}), 7.13-7.29(\mathrm{~m}, 4 \mathrm{H}), 7.74(\mathrm{dd}, J=6.5,2.0 \mathrm{~Hz}, 1 \mathrm{H})$, and $8.62(\mathrm{dd}, J=$ 8.0, $1.5 \mathrm{~Hz}, 1 \mathrm{H}) ;{ }^{13} \mathrm{C}\left\{{ }^{1} \mathrm{H}\right\}$ NMR $\left(101 \mathrm{MHz}, \mathrm{CDCl}_{3}\right) \delta 26.13,34.94,47.54,86.96,109.1,119.5$, 119.7, 120.4, 122.6, 122.7, 123.0, $131.3(2 \times \mathrm{C}), 136.5,142.8,150.1$, and 157.4; IR $(\mathrm{KBr}) v_{\max }$ $1611,1578,1477,1441,1388,1328,1308$, and $1228 \mathrm{~cm}^{-1}$; MS (ESI) $\mathrm{m} / z$ (rel intensity) 293 (100\%, $\left.\mathrm{MH}^{+}\right)$; HRMS calcd for $\mathrm{C}_{19} \mathrm{H}_{21} \mathrm{~N}_{2} \mathrm{O}\left(\mathrm{MH}^{+}\right)$293.1654, found 293.1640; Anal. Calcd for $\mathrm{C}_{19} \mathrm{H}_{20} \mathrm{~N}_{2} \mathrm{O}: \mathrm{C}, 78.05 ; \mathrm{H}, 6.89 ; \mathrm{N}, 9.58$. Found: C, 77.70; H, 6.97; N, 9.62.

Single crystals of compound 9 suitable for $\mathrm{X}$-ray analysis were grown by slow evaporation of its $\mathrm{CH}_{2} \mathrm{Cl}_{2}$-EtOAc solution (see: Chapter 3, for data).

\section{6-tert-Butyl-11-methyl-6,7-dihydro-5-oxa-7a,12-diazadibenzo[a,e]azulene (11)}

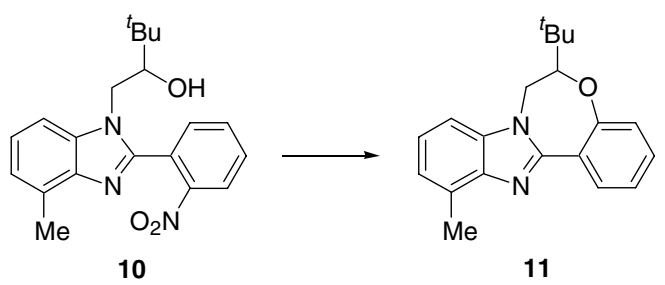

To a solution of alcohol $10(100 \mathrm{mg}, 0.28 \mathrm{mmol})$ in anhydrous DMF (2 mL) was added $\mathrm{NaH}(60 \% \mathrm{w} / \mathrm{w}, 12.5 \mathrm{mg}, 0.31 \mathrm{mmol})$ to give a dark-brown solution. After $13 \mathrm{~h}$ at $\mathrm{rt}$, the reaction mixture was quenched with water, and diluted with EtOAc. The organic phase was washed repeatedly with water, dried $\left(\mathrm{MgSO}_{4}\right)$, and evaporated in vacuo to give a yellow oil. Purification by flash chromatography (silica gel, $\mathrm{CH}_{2} \mathrm{Cl}_{2} \rightarrow \mathrm{CH}_{2} \mathrm{Cl}_{2} / \mathrm{EtOAc}, 5 / 1$ ) gave the title compound 11 (83 mg, 96\%) as a clear oil that solidified on standing: $\mathrm{R}_{f}=0.55$ (petroleum ether/EtOAc, 9/1); ${ }^{1} \mathrm{H}$ NMR (400 MHz, $\left.\mathrm{CDCl}_{3}\right) \delta 1.07$ (s, 9H), 2.65 (s, 3H), 3.79 (dd, J = 9.5, 1.0 Hz, 1H), 4.15 (dd, $J=13.5,9.5 \mathrm{~Hz}, 1 \mathrm{H}), 4.45(\mathrm{dd}, J=13.5,1.0 \mathrm{~Hz}, 1 \mathrm{H}), 6.96-7.12(\mathrm{~m}, 5 \mathrm{H}), 7.24$ (dt, $J=7.5,1.5$ $\mathrm{Hz}, 1 \mathrm{H})$, and $8.67(\mathrm{dd}, J=8.0,1.5 \mathrm{~Hz}, 1 \mathrm{H}) ;{ }^{13} \mathrm{C}\left\{{ }^{1} \mathrm{H}\right\} \mathrm{NMR}\left(101 \mathrm{MHz}, \mathrm{CDCl}_{3}\right) \delta 17.10,26.58$, 35.37, 47.97, 87.53, 107.0, 120.3, 120.8, 123.0, 123.4 (2 × C), 130.4, 131.5, 131.9, 136.7, 142.7, 149.7, and 157.6; IR $\left(\mathrm{CHCl}_{3}\right) v_{\max } 2965,1603,1576,1475,1446,1373$, and $1273 \mathrm{~cm}^{-1}$; MS (ESI) $m / z$ (rel intensity) $307\left(100 \%, \mathrm{MH}^{+}\right)$; HRMS calcd for $\mathrm{C}_{20} \mathrm{H}_{23} \mathrm{~N}_{2} \mathrm{O}\left(\mathrm{MH}^{+}\right)$307.1810, found 307.1817 . 


\section{Ethyl 6-tert-butyl-6,7-dihydro-5-oxa-7a,12-diazadibenzo[a,e]azulene-11-carboxylate (13)}

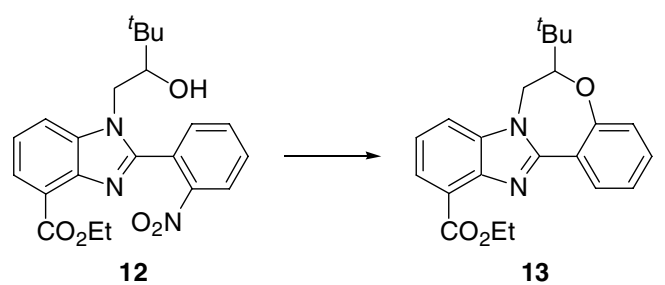

To a solution of alcohol $\mathbf{1 2}^{8}(100 \mathrm{mg}, 0.24 \mathrm{mmol})$ in anhydrous DMF (2 mL) was added $\mathrm{NaH}(60 \% \mathrm{w} / \mathrm{w}, 10.8 \mathrm{mg}, 0.27 \mathrm{mmol})$. After $20 \mathrm{~h}$ at $\mathrm{rt}$, the reaction mixture was quenched with water, and diluted with EtOAc. The organic phase was washed repeatedly with water, dried $\left(\mathrm{MgSO}_{4}\right)$, and evaporated in vacuo to give a clear oil. Purification by flash chromatography (silica gel, $\mathrm{CH}_{2} \mathrm{Cl}_{2} \rightarrow \mathrm{CH}_{2} \mathrm{Cl}_{2} / \mathrm{EtOAc}$, 4/1) gave the title compound 13 (79 $\mathrm{mg}, 89 \%$ ) as a clear oil that solidified on standing: $\mathrm{R}_{f}=0.55$ (petroleum ether/EtOAc, 3/1); ${ }^{1} \mathrm{H}$ NMR (250 MHz, $\left.\mathrm{CDCl}_{3}\right) \delta 1.20(\mathrm{~s}, 9 \mathrm{H}), 1.54(\mathrm{t}, J=7.0 \mathrm{~Hz}, 3 \mathrm{H}), 3.91(\sim \mathrm{d}, J=9.5 \mathrm{~Hz}, 1 \mathrm{H}), 4.32(\mathrm{dd}, J=13.5,9.5$ $\mathrm{Hz}, 1 \mathrm{H}), 4.56(\mathrm{q}, J=7.0 \mathrm{~Hz}, 2 \mathrm{H}), 4.61(\mathrm{~d}, J=13.5 \mathrm{~Hz}, 1 \mathrm{H}), 7.11(\mathrm{~d}, J=8.0 \mathrm{~Hz}, 1 \mathrm{H}), 7.22(\mathrm{dt}, J$ $=7.0,1.0 \mathrm{~Hz}, 1 \mathrm{H}), 7.28-7.44(\mathrm{~m}, 2 \mathrm{H}), 7.53(\mathrm{~d}, J=8.0 \mathrm{~Hz}, 1 \mathrm{H}), 8.01(\mathrm{~d}, J=7.5 \mathrm{~Hz}, 1 \mathrm{H})$, and $8.89(\mathrm{dd}, J=8.0,1.5 \mathrm{~Hz}, 1 \mathrm{H}) ;{ }^{13} \mathrm{C}\left\{{ }^{1} \mathrm{H}\right\} \mathrm{NMR}\left(63 \mathrm{MHz}, \mathrm{CDCl}_{3}\right) \delta 14.92,26.56,35.37,48.29$, $61.41,87.18,114.0,119.6,120.6,121.7,122.1$, 123.5, 126.0, 132.2, 132.5, 138.3 , 142.4, 152.1, 158.0, and 166.7; IR $\left(\mathrm{CHCl}_{3}\right) v_{\max } 2966,1708,1608,1476,1427,1299$, and $1256 \mathrm{~cm}^{-1}$; MS (ESI) $m / z$ (rel intensity) $365\left(100 \%, \mathrm{MH}^{+}\right.$), 337 (35), and 319 (20); HRMS calcd for $\mathrm{C}_{22} \mathrm{H}_{24} \mathrm{~N}_{2} \mathrm{NaO}_{3}\left(\mathrm{MNa}^{+}\right)$387.1685, found 387.1692.

\section{6-tert-Butyl-11-[1,3]dioxolan-2-yl-6,7-dihydro-5-oxa-7a,12-diazadibenzo[a,e ]azulene (3)}
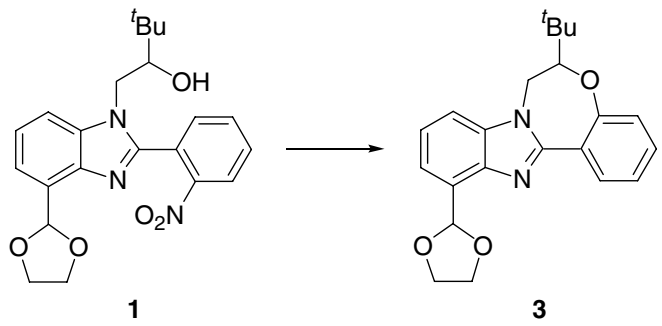

To a solution of alcohol $\mathbf{1}^{8}$ (200 $\left.\mathrm{mg}, 0.49 \mathrm{mmol}\right)$ in anhydrous DMF (2 mL) was added $\mathrm{NaH}(60 \% \mathrm{w} / \mathrm{w}, 21.6 \mathrm{mg}, 0.54 \mathrm{mmol})$. After $19 \mathrm{~h}$ at $\mathrm{rt}$, the reaction mixture was quenched with water, and diluted with EtOAc. The organic phase was washed repeatedly with water, dried $\left(\mathrm{MgSO}_{4}\right)$, and evaporated in vacuo to give an off-white solid. Purification by flash chromatography (silica gel, $\mathrm{CH}_{2} \mathrm{Cl}_{2} \rightarrow \mathrm{CH}_{2} \mathrm{Cl}_{2} / \mathrm{EtOAc}, 10 / 1$ ) gave the title compound 3 (161 $\mathrm{mg}$, $92 \%$ ) as a white solid: $\mathrm{R}_{f}=0.35$ (petroleum ether/EtOAc, 3/1); $\mathrm{mp}$ 200.0-201.0 ${ }^{\circ} \mathrm{C}$ 
(EtOAc/petroleum ether); ${ }^{1} \mathrm{H}$ NMR $\left(250 \mathrm{MHz}, \mathrm{CDCl}_{3}\right) \delta 1.13(\mathrm{~s}, 9 \mathrm{H}), 3.84(\mathrm{dd}, J=9.0,1.0 \mathrm{~Hz}$, $1 \mathrm{H}), 4.05-4.32(\mathrm{~m}, 5 \mathrm{H}), 4.52(\mathrm{dd}, J=13.5,1.0 \mathrm{~Hz}, 1 \mathrm{H}), 6.65(\mathrm{~s}, 1 \mathrm{H}), 7.04(\mathrm{dd}, J=8.0,1.0 \mathrm{~Hz}$, $1 \mathrm{H}), 7.12(\mathrm{dt}, J=8.0,1.0 \mathrm{~Hz}, 1 \mathrm{H}), 7.19-7.35(\mathrm{~m}, 3 \mathrm{H}), 7.46(\mathrm{dd}, J=6.5,1.5 \mathrm{~Hz}, 1 \mathrm{H})$, and 8.75 $(\mathrm{dd}, J=8.0,1.5 \mathrm{~Hz}, 1 \mathrm{H}) ;{ }^{13} \mathrm{C}\left\{{ }^{1} \mathrm{H}\right\} \mathrm{NMR}\left(63 \mathrm{MHz}, \mathrm{CDCl}_{3}\right) \delta 26.57,35.35,48.01,66.07$ and 66.11 (rotamers?), 87.44, 101.3, 110.4, 120.0, 120.2, 120.7, 122.8, 123.3, 129.1, 131.7, 132.4, 137.4, 141.7, 150.8, and 157.7; IR $\left(\mathrm{CHCl}_{3}\right) v_{\max } 2965,1610,1576,1475,1437$, and $1076 \mathrm{~cm}^{-1}$; MS (ESI) $m / z$ (rel intensity) 365 (100\%, $\mathrm{MH}^{+}$) and 321 (15); HRMS calcd for $\mathrm{C}_{20} \mathrm{H}_{25} \mathrm{~N}_{2} \mathrm{O}_{3}$ $\left(\mathrm{MH}^{+}\right)$365.1865, found 365.1870; Anal. Calcd for $\mathrm{C}_{22} \mathrm{H}_{24} \mathrm{~N}_{2} \mathrm{O}_{3}: \mathrm{C}, 72.50 ; \mathrm{H}, 6.64 ; \mathrm{N}, 7.69$. Found: C, 72.34; H, 6.68; N, 7.61. The cyclic ether $\mathbf{3}$ was optically resolved by CSP HPLC (Figure S1).

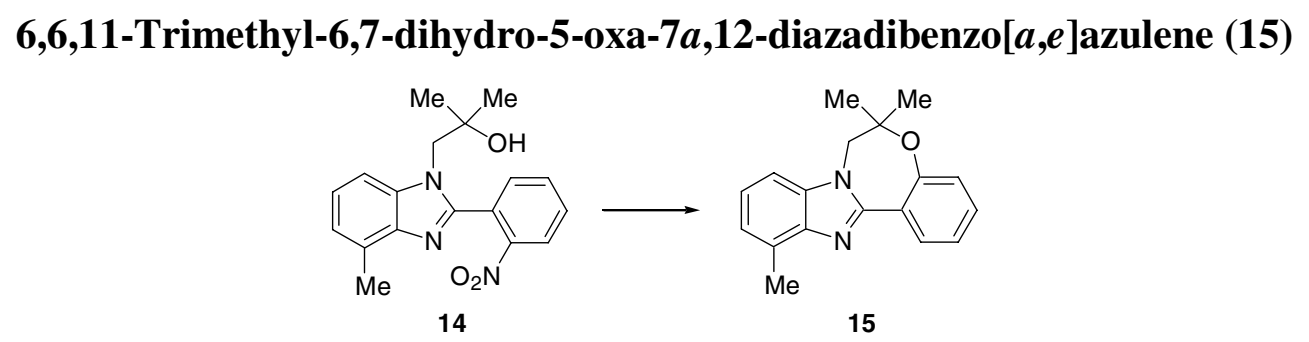

To a solution of alcohol 14 (100 mg, $0.31 \mathrm{mmol})$ in anhydrous DMF (1 mL) was added $\mathrm{NaH}(95 \% \mathrm{w} / \mathrm{w}, 8.6 \mathrm{mg}, 0.34 \mathrm{mmol})$ to give a brown solution. After $2 \mathrm{~h}$ at $\mathrm{rt}$, the reaction mixture was quenched with water, and diluted with EtOAc. The organic phase was washed repeatedly with water, dried $\left(\mathrm{MgSO}_{4}\right)$, and evaporated in vacuo to give a pale brown oil. Purification by flash chromatography (silica gel, $\mathrm{CH}_{2} \mathrm{Cl}_{2} \rightarrow \mathrm{CH}_{2} \mathrm{Cl}_{2} / \mathrm{EtOAc}$, 8/1) gave the title compound 15 (58 mg, 68\%) as a clear oil: $\mathrm{R}_{f}=0.40$ (petroleum ether/EtOAc, 3/1); ${ }^{1} \mathrm{H}$ NMR (400 $\left.\mathrm{MHz} \mathrm{CDCl}_{3}\right) \delta 1.48(\mathrm{~s}, 6 \mathrm{H}), 2.79(\mathrm{~s}, 3 \mathrm{H}), 4.05(\mathrm{~s}, 2 \mathrm{H}), 7.09-7.32(\mathrm{~m}, 5 \mathrm{H}), 7.45(\sim \mathrm{t}, J=8.0 \mathrm{~Hz}$, $1 \mathrm{H})$, and $8.20(\mathrm{~d}, J=7.5 \mathrm{~Hz}, 1 \mathrm{H}) ;{ }^{13} \mathrm{C}\left\{{ }^{1} \mathrm{H}\right\} \mathrm{NMR}\left(63 \mathrm{MHz}, \mathrm{CDCl}_{3}\right) \delta 17.31,25.64,52.12,84.76$, 106.5, 123.1 ( $2 \times \mathrm{C}$ ?), 124.1, 124.2, 124.6, 130.6, 130.8, 131.9, 135.9, 143.0, 151.8, and 153.7; IR $\left(\mathrm{CHCl}_{3}\right) v_{\max } 1610,1469,1455$, and $1386 \mathrm{~cm}^{-1}$; MS (ESI) $\mathrm{m} / z$ (rel intensity) 301 (90\%, $\mathrm{MNa}^{+}$) and 279 (100); HRMS calcd for $\mathrm{C}_{18} \mathrm{H}_{19} \mathrm{~N}_{2} \mathrm{O}\left(\mathrm{MH}^{+}\right)$279.1497, found 279.1494. 


\section{6-tert-Butyl-4-chloro-11-methyl-6,7-dihydro-5-oxa-7a,12-diazadibenzo[a,e]azulene (17)}

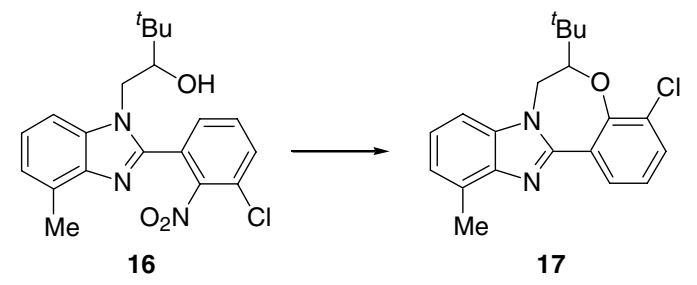

To a solution of alcohol 16 (100 mg, $0.26 \mathrm{mmol})$ in anhydrous DMF (2 mL) was added $\mathrm{NaH}(60 \% \mathrm{w} / \mathrm{w}, 11.5 \mathrm{mg}, 0.29 \mathrm{mmol})$. After $23 \mathrm{~h}$ at $\mathrm{rt}$, the reaction mixture was quenched with water, and diluted with EtOAc. The organic phase was washed repeatedly with water, dried $\left(\mathrm{MgSO}_{4}\right)$, and evaporated in vacuo to give a white foam. Purification by flash chromatography (silica gel, $\left.\mathrm{CH}_{2} \mathrm{Cl}_{2} \rightarrow \mathrm{CH}_{2} \mathrm{Cl}_{2} / \mathrm{EtOAc}, 10 / 1\right)$ gave the title compound 17 (70 $\mathrm{mg}, 80 \%$ ) as a white solid: $\mathrm{R}_{f}=0.55$ (petroleum ether/EtOAc, 9/1); mp 191.0-192.0 ${ }^{\circ} \mathrm{C}$ (EtOAc/petroleum ether); ${ }^{1} \mathrm{H}$ NMR (400 MHz, CDCl $) \delta 1.24(\mathrm{~s}, 9 \mathrm{H}), 2.74(\mathrm{~s}, 3 \mathrm{H}), 3.98(\mathrm{~d}, J=8.5 \mathrm{~Hz}, 1 \mathrm{H}), 4.26(\mathrm{dd}, J=$ 14.0, 8.5 Hz, 1H), $4.57(\mathrm{~d}, J=14.0 \mathrm{~Hz}, 1 \mathrm{H}), 7.05-7.25(\mathrm{~m}, 4 \mathrm{H}), 7.45$ (dd, $J=7.5,1.5 \mathrm{~Hz}, 1 \mathrm{H})$, and $8.68(\mathrm{dd}, J=8.0,1.5 \mathrm{~Hz}, 1 \mathrm{H}) ;{ }^{13} \mathrm{C}\left\{{ }^{1} \mathrm{H}\right\} \mathrm{NMR}\left(101 \mathrm{MHz}, \mathrm{CDCl}_{3}\right) \delta 16.90,26.62,35.25$, 47.15, 88.78, 106.8, 121.2, 123.1 (2 × C), 123.3, 126.0, 130.3, 130.4, 131.9, 136.3, 142.5, 148.8, and 152.3; IR $\left(\mathrm{CHCl}_{3}\right) v_{\max } 2965,1468,1422$, and $1369 \mathrm{~cm}^{-1}$; MS (ESI) $\mathrm{m} / z$ (rel intensity) 341 (100\%, $\mathrm{MH}^{+}$); HRMS calcd for $\mathrm{C}_{20} \mathrm{H}_{22} \mathrm{ClN}_{2} \mathrm{O}\left(\mathrm{MH}^{+}\right)$341.1420, found 341.1407; Anal. Calcd for $\mathrm{C}_{20} \mathrm{H}_{21} \mathrm{ClN}_{2} \mathrm{O}$ : C, 70.48; H, 6.21; Cl, 10.40; N, 8.22. Found: C, 70.43; H, 6.18; Cl, 10.53; N, 8.26 .

6-tert-Butyl-4,11-dimethyl-6,7-dihydro-5-oxa-7a,12-diazadibenzo[a,e]azulene (19)

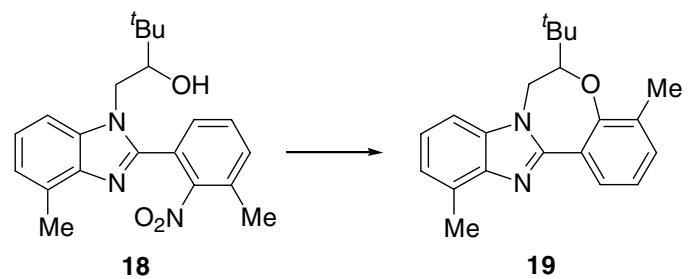

To a solution of alcohol 18 (200 mg, $0.54 \mathrm{mmol})$ in anhydrous DMF (2 mL) was added $\mathrm{NaH}(95 \% \mathrm{w} / \mathrm{w}, 15.1 \mathrm{mg}, 0.6 \mathrm{mmol})$ to give a deep-violet solution. After $22 \mathrm{~h}$ at $\mathrm{rt}$, the reaction mixture was quenched with water, and diluted with EtOAc. The organic phase was washed repeatedly with water, dried $\left(\mathrm{MgSO}_{4}\right)$, and evaporated in vacuo to give a brown oil. Purification by flash chromatography (silica gel, $\mathrm{CH}_{2} \mathrm{Cl}_{2} \rightarrow \mathrm{CH}_{2} \mathrm{Cl}_{2} / \mathrm{EtOAc}, 10 / 1$ ) gave the title compound 19 $(13 \mathrm{mg}, 7 \%)$ as a white solid: $\mathrm{R}_{f}=0.55$ (petroleum ether/EtOAc, 9/1); ${ }^{1} \mathrm{H}$ NMR (400 MHz, 
$\left.\mathrm{CDCl}_{3}\right) \delta 1.18(\mathrm{~s}, 9 \mathrm{H}), 2.37(\mathrm{~s}, 3 \mathrm{H}), 2.73(\mathrm{~s}, 3 \mathrm{H}), 3.92(\mathrm{~d}, J=7.5 \mathrm{~Hz}, 1 \mathrm{H}), 4.22(\mathrm{dd}, J=14.0,7.5$ $\mathrm{Hz}, 1 \mathrm{H}), 4.55(\mathrm{~d}, J=14.0 \mathrm{~Hz}, 1 \mathrm{H}), 7.03-7.22(\mathrm{~m}, 5 \mathrm{H})$, and $8.55(\mathrm{~d}, J=8.0 \mathrm{~Hz}, 1 \mathrm{H}),{ }^{13} \mathrm{C}\left\{{ }^{1} \mathrm{H}\right\}$ NMR $\left(101 \mathrm{MHz}, \mathrm{CDCl}_{3}\right) \delta 16.73,17.36,26.44,34.92,46.64,87.49,106.3,118.4,122.2,122.4$, $122.9,129.3,129.5,129.9,132.6,135.8,142.2,150.1$, and 154.5; $\mathrm{IR}\left(\mathrm{CHCl}_{3}\right) v_{\max } 2965,1601$, 1475, and $1417 \mathrm{~cm}^{-1}$; MS (ESI) $\mathrm{m} / z$ (rel intensity) $321\left(100 \%, \mathrm{MH}^{+}\right.$); HRMS calcd for $\mathrm{C}_{21} \mathrm{H}_{24} \mathrm{~N}_{2} \mathrm{NaO}\left(\mathrm{MNa}^{+}\right)$343.1786, found 343.1772.

6-tert-Butyl-11-methyl-6,7-dihydro-5-oxa-7a,12-diazadibenzo[a,e $]$ azulene (11)

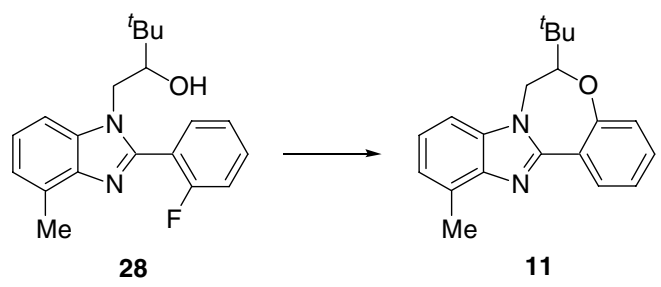

To a solution of alcohol 28 (100 mg, $0.31 \mathrm{mmol})$ in anhydrous DMF (2 mL) was added $\mathrm{NaH}(60 \% \mathrm{w} / \mathrm{w}, 13.5 \mathrm{mg}, 0.34 \mathrm{mmol})$. After $5 \mathrm{~h}$ at $\mathrm{rt}$, the reaction mixture was quenched with water, and diluted with EtOAc. The organic phase was washed repeatedly with water, dried $\left(\mathrm{MgSO}_{4}\right)$, and evaporated in vacuo. Purification by flash chromatography (silica gel, $\mathrm{CH}_{2} \mathrm{Cl}_{2} \rightarrow$ $\left.\mathrm{CH}_{2} \mathrm{Cl}_{2} / \mathrm{EtOAc}, 5 / 1\right)$ gave the title compound $11(87 \mathrm{mg}, 93 \%)$ identical (TLC, ${ }^{1} \mathrm{H}$ NMR) with the product of the nitro-group displacement from alcohol $\mathbf{1 0}$ (vide supra).

\section{Attempted Replacement of the Chloro Group in Alcohol (29)}

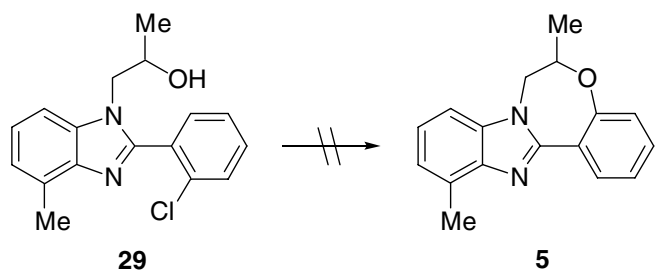

To a solution of alcohol 29 (136 mg, $0.45 \mathrm{mmol})$ in anhydrous DMF (2 mL) was added $\mathrm{NaH}(95 \% \mathrm{w} / \mathrm{w}, 12.6 \mathrm{mg}, 0.50 \mathrm{mmol})$. After $24 \mathrm{~h}$ at $\mathrm{rt}$, the reaction mixture was quenched with water, neutralized with $5 \% \mathrm{HCl}$, and diluted with EtOAc. The organic phase was washed repeatedly with water, dried $\left(\mathrm{MgSO}_{4}\right)$, and evaporated in vacuo. ${ }^{1} \mathrm{H}$ NMR analysis of the crude product indicated the presence of the starting material 29, only. Purification by flash chromatography (silica gel, $\mathrm{CH}_{2} \mathrm{Cl}_{2} \rightarrow$ EtOAc) gave the recovered starting material 29 (107 mg, $79 \%)$. 


\section{(E/Z)-2-(4-Methyl-1-propenyl-1H-benzimidazol-2-yl)phenol (30)}

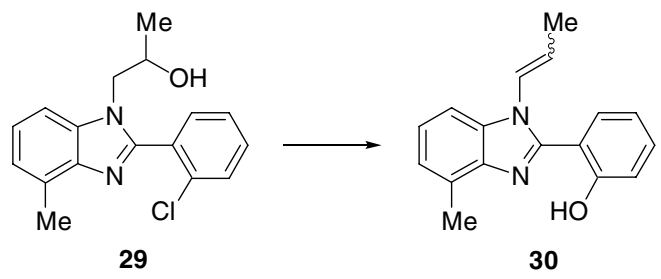

\section{Method 1:}

To a solution of alcohol 29 (107 mg, $0.36 \mathrm{mmol})$ in anhydrous DMF (1 mL) was added $\mathrm{NaH}(95 \% \mathrm{w} / \mathrm{w}, 9.9 \mathrm{mg}, 0.4 \mathrm{mmol})$. After $24 \mathrm{~h}$ at $90{ }^{\circ} \mathrm{C}$, the reaction mixture was quenched with water, neutralized with $5 \% \mathrm{HCl}$, and diluted with EtOAc. The organic phase was washed repeatedly with water, dried $\left(\mathrm{MgSO}_{4}\right)$, and evaporated in vacuo to give a pale brown oil. Purification by flash chromatography (silica gel, $\mathrm{CH}_{2} \mathrm{Cl}_{2} \rightarrow$ EtOAc) gave the title compound 30 (37 mg, 39\%, E/Z = 2:1), cyclic ether 5 ( 3 mg, 3\%), and recovered starting material 29 (45 mg, 42\%). Phenol 30: a white solid, MS (ESI) $\mathrm{m} / \mathrm{z}$ (rel intensity) $287\left(60 \%, \mathrm{MNa}^{+}\right.$) and 265 (100); HRMS calcd for $\mathrm{C}_{17} \mathrm{H}_{16} \mathrm{~N}_{2} \mathrm{NaO}\left(\mathrm{MNa}^{+}\right)$287.1160, found 287.1158.

Method 2:

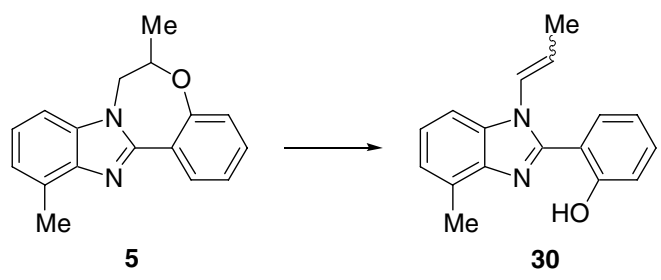

To a solution of the cyclic ether $5(54 \mathrm{mg}, 0.20 \mathrm{mmol})$ in anhydrous DMF (1 mL) was added $\mathrm{NaH}(95 \% \mathrm{w} / \mathrm{w}, 5.7 \mathrm{mg}, 0.2 \mathrm{mmol})$. After $18 \mathrm{~h}$ at $90{ }^{\circ} \mathrm{C}$, the reaction mixture was quenched with water, neutralized with $5 \% \mathrm{HCl}$, and diluted with EtOAc. The organic phase was washed repeatedly with water, dried $\left(\mathrm{MgSO}_{4}\right)$, and evaporated in vacuo to give an off-white solid. Purification by flash chromatography (silica gel, $\mathrm{CH}_{2} \mathrm{Cl}_{2} \rightarrow$ EtOAc) gave the title compound 30 (46 $\mathrm{mg}, 85 \%, E / Z=\sim 2: 1)$ as a white solid.

\section{Method 3:}

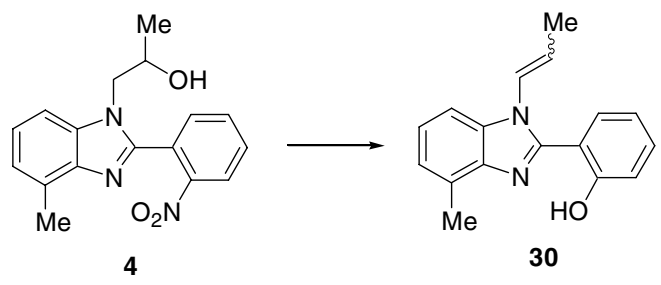


To a solution of alcohol 4 (100 mg, $0.32 \mathrm{mmol})$ in anhydrous DMF (1 mL) was added $\mathrm{NaH}(95 \% \mathrm{w} / \mathrm{w}, 17.9 \mathrm{mg}, 0.7 \mathrm{mmol})$. After $1 \mathrm{~h}$ at $\mathrm{rt}$, followed by $23 \mathrm{~h}$ at $90{ }^{\circ} \mathrm{C}$, the reaction mixture was quenched with water, neutralized with $5 \% \mathrm{HCl}$, and diluted with EtOAc. The organic phase was washed repeatedly with water, dried $\left(\mathrm{MgSO}_{4}\right)$, and evaporated in vacuo to give a brown oil. Purification by flash chromatography (silica gel, $\mathrm{CH}_{2} \mathrm{Cl}_{2}$ ) gave the title compound $30(60 \mathrm{mg}, 71 \%, E / Z=\sim 2.2: 1)$ as a white solid. The two isomeric products were separated by repeated purification by flash chromatography (silica gel, petroleum ether/ $\mathrm{CH}_{2} \mathrm{Cl}_{2}$, 1/1). Phenol (Z)-30: a white solid: $\mathrm{R}_{f}=0.60$ (petroleum ether/ $\left.\mathrm{CH}_{2} \mathrm{Cl}_{2}, 1 / 1\right)$; ${ }^{1} \mathrm{H}$ NMR $(400 \mathrm{MHz}$, $\left.\mathrm{CDCl}_{3}\right) \delta 1.58(\mathrm{dd}, J=7.0,2.0 \mathrm{~Hz}, 3 \mathrm{H}), 2.67(\mathrm{~s}, 3 \mathrm{H}), 6.15(\mathrm{dq}, J=8.0,7.0 \mathrm{~Hz}, 1 \mathrm{H}), 6.83(\mathrm{dq}, J=$ 8.0, $2.0 \mathrm{~Hz}, 1 \mathrm{H}), 6.87$ ( dt, $J=7.5,1.0 \mathrm{~Hz}, 1 \mathrm{H}), 7.05(\mathrm{~d}, J=8.0 \mathrm{~Hz}, 1 \mathrm{H}), 7.11$ (dd, $J=8.5,1.0$ $\mathrm{Hz}, 1 \mathrm{H}), 7.12(\sim \mathrm{dd}, J=7.5,1.0 \mathrm{~Hz}, 1 \mathrm{H}), 7.21(\mathrm{t}, J=8.0 \mathrm{~Hz}, 1 \mathrm{H}), 7.32(\mathrm{ddd}, J=8.5,7.5,1.5 \mathrm{~Hz}$, $1 \mathrm{H}), 8.08(\mathrm{dd}, J=8.5,1.5 \mathrm{~Hz}, 1 \mathrm{H})$, and 13.7 (br s, $1 \mathrm{H}) ;{ }^{13} \mathrm{C}\left\{{ }^{1} \mathrm{H}\right\} \mathrm{NMR}\left(101 \mathrm{MHz}, \mathrm{CDCl}_{3}\right) \delta$ 12.92 , 16.54, 108.2, 113.4, 117.9, 118.3, 123.6, 124.6, 126.8, 128.9, 129.3, 131.5, 134.0, 139.4, 149.9, and 159.5 (one $\mathrm{C}$ atom obscured); IR $\left(\mathrm{CHCl}_{3}\right) v_{\max } 1623,1583,1484,1375,1363,1271$, and $1255 \mathrm{~cm}^{-1}$; MS (ESI) $\mathrm{m} / z$ (rel intensity) $265\left(100 \%, \mathrm{MH}^{+}\right.$) and 225 (90); HRMS calcd for $\mathrm{C}_{17} \mathrm{H}_{17} \mathrm{~N}_{2} \mathrm{O}\left(\mathrm{MH}^{+}\right)$265.1341, found 265.1330. Phenol $(E)-30$ : a white solid: $\mathrm{R}_{f}=0.55$ (petroleum ether/ $\left.\mathrm{CH}_{2} \mathrm{Cl}_{2}, 1 / 1\right) ;{ }^{1} \mathrm{H}$ NMR $\left(400 \mathrm{MHz}, \mathrm{CDCl}_{3}\right) \delta 2.04(\mathrm{dd}, J=7.0,1.5 \mathrm{~Hz}, 3 \mathrm{H}), 2.66(\mathrm{~s}, 3 \mathrm{H})$, $6.19(\mathrm{dq}, J=14.0,7.0 \mathrm{~Hz}, 1 \mathrm{H}), 6.80(\sim \mathrm{dd}, J=14.0,1.5 \mathrm{~Hz}, 1 \mathrm{H}), 6.91$ (t, $J=7.5 \mathrm{~Hz}, 1 \mathrm{H}), 7.09$ $7.16(\mathrm{~m}, 2 \mathrm{H}), 7.20(\mathrm{t}, J=7.5 \mathrm{~Hz}, 1 \mathrm{H}), 7.28(\mathrm{~d}, J=8.0 \mathrm{~Hz}, 1 \mathrm{H}), 7.34(\mathrm{dt}, J=8.0,1.5 \mathrm{~Hz}, 1 \mathrm{H})$, $7.93(\mathrm{~d}, J=8.0 \mathrm{~Hz}, 1 \mathrm{H})$, and $13.4($ br s, $1 \mathrm{H}) ;{ }^{13} \mathrm{C}\left\{{ }^{1} \mathrm{H}\right\} \mathrm{NMR}\left(101 \mathrm{MHz}, \mathrm{CDCl}_{3}\right) \delta 15.36,16.50$, $108.1,113.3$, 117.9, 118.3, 123.5, 123.6, 125.1, 127.3, 127.5, 128.8, 131.4, 134.5, 139.7, 149.7, and 159.3; IR $\left(\mathrm{CHCl}_{3}\right) v_{\max } 1624,1584,1485,1385,1373,1270$, and $1256 \mathrm{~cm}^{-1}$; MS (ESI) $\mathrm{m} / z$ (rel intensity) $265\left(100 \%, \mathrm{MH}^{+}\right.$) and 225 (60); HRMS calcd for $\mathrm{C}_{17} \mathrm{H}_{17} \mathrm{~N}_{2} \mathrm{O}\left(\mathrm{MNH}^{+}\right)$265.1341, found 265.1323 . 


\subsection{Intramolecular $S_{N} A r$ Reactions with Diols}

\section{(6,7-Dihydro-5-oxa-7a,12-diazadibenzo[a,e]azulen-6-yl)methanol (22)}

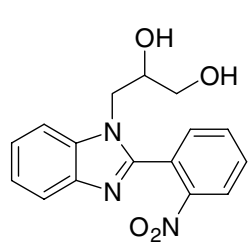

20

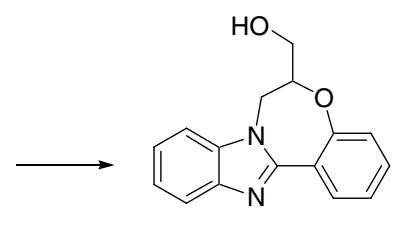

22

To a solution of diol 20 (1.00 g, $3.19 \mathrm{mmol})$ in anhydrous DMF $(10 \mathrm{~mL})$ was added $\mathrm{NaH}$ $(60 \% \mathrm{w} / \mathrm{w}, 281 \mathrm{mg}, 7.0 \mathrm{mmol})$ to give a dark-brown solution. After $100 \mathrm{~min}$ at $\mathrm{rt}$, the reaction mixture was quenched with water, and diluted with EtOAc. The organic phase was washed repeatedly with water, dried $\left(\mathrm{MgSO}_{4}\right)$, and evaporated in vacuo to give an off-white solid. Purification by flash chromatography (silica gel, $\mathrm{CH}_{2} \mathrm{Cl}_{2} \rightarrow \mathrm{CH}_{2} \mathrm{Cl}_{2} / \mathrm{EtOAc}, 1 / 1$ ) gave the title compound $22(818 \mathrm{mg}, 96 \%)$ as a white solid: $\mathrm{R}_{f}=0.65$ (EtOAc); $\mathrm{mp}$ 142.0-143.5 ${ }^{\circ} \mathrm{C}$ (EtOAc/petroleum ether); $[\alpha]_{\mathrm{D}}=-0.7$ (c 0.88 in $\left.\mathrm{CHCl}_{3}\right) ;{ }^{1} \mathrm{H}$ NMR $\left(400 \mathrm{MHz}, \mathrm{CDCl}_{3}\right) \delta 3.45$ (br s, $1 \mathrm{H}), 3.98(\mathrm{dd}, J=11.5,5.0 \mathrm{~Hz}, 1 \mathrm{H}), 4.04(\mathrm{dd}, J=11.5,5.0 \mathrm{~Hz}, 1 \mathrm{H}), 4.35(\mathrm{dd}, J=13.0,8.5$ $\mathrm{Hz}, 1 \mathrm{H}), 4.39-4.44(\mathrm{~m}, 1 \mathrm{H}), 4.55$ (dd, $J=13.0,0.5 \mathrm{~Hz}, 1 \mathrm{H}), 7.05$ (dd, $J=8.0,1.0 \mathrm{~Hz}, 1 \mathrm{H}), 7.20$ $(\mathrm{dt}, J=8.0,1.0 \mathrm{~Hz}, 1 \mathrm{H}), 7.28-7.37(\mathrm{~m}, 4 \mathrm{H}), 7.84-7.86(\mathrm{~m}, 1 \mathrm{H})$, and $8.60(\mathrm{dd}, J=8.0,1.5 \mathrm{~Hz}$, $1 \mathrm{H}) ;{ }^{13} \mathrm{C}\left\{{ }^{1} \mathrm{H}\right\} \mathrm{NMR}\left(63 \mathrm{MHz}, \mathrm{CDCl}_{3}\right) \delta 47.37,63.15,80.17,109.2,119.3,119.5,121.0,122.9$, 123.0, 123.5, 131.2, 131.5, 136.1, 142.5, 150.1, and 155.7; IR $\left(\mathrm{CHCl}_{3}\right) v_{\max } 2963,1611,1576$, 1476, 1445, 1387, and $1324 \mathrm{~cm}^{-1}$; MS (ESI) $\mathrm{m} / \mathrm{z}$ (rel intensity) $267\left(100 \%, \mathrm{MH}^{+}\right.$); HRMS calcd for $\mathrm{C}_{16} \mathrm{H}_{15} \mathrm{~N}_{2} \mathrm{O}_{2}\left(\mathrm{MH}^{+}\right)$267.1133, found 267.1122; Anal. Calcd for $\mathrm{C}_{16} \mathrm{H}_{14} \mathrm{~N}_{2} \mathrm{O}_{2}$ : C, 72.16; $\mathrm{H}$, 5.30; N, 10.52. Found: C, 72.00; H, 5.19; N, 10.55. Alcohol 22 was optically resolved by CSP HPLC (Figure S2).

(6,7-Dihydro-5-oxa-7a,12-diazadibenzo[a,e]azulen-6-yl)methanol (22) and 6,7-dihydro-5-oxa-7a,12-diazadibenzo[a,e]azulen-6-ylmethyl acetate (58)

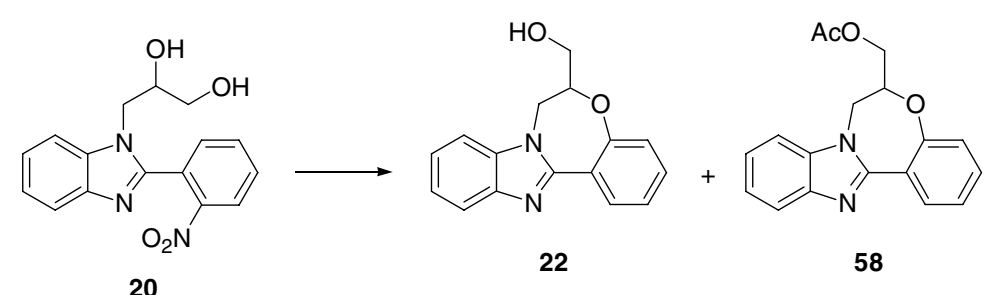


To a solution of diol $20(1.00 \mathrm{~g}, 3.19 \mathrm{mmol})$ in anhydrous DMF $(10 \mathrm{~mL})$ was added $\mathrm{NaH}$ $(60 \% \mathrm{w} / \mathrm{w}, 281 \mathrm{mg}, 7.0 \mathrm{mmol})$ to give a dark-brown solution. After $2 \mathrm{~h}$ at $\mathrm{rt}$, the reaction mixture was diluted with EtOAc, and washed repeatedly with water. The organic layer was dried $\left(\mathrm{MgSO}_{4}\right)$, and evaporated in vacuo to give an off-white oil. Purification by flash chromatography (silica gel, $\left.\mathrm{CH}_{2} \mathrm{Cl}_{2} \rightarrow \mathrm{CH}_{2} \mathrm{Cl}_{2} / \mathrm{EtOAc}, 1 / 1\right)$ gave the title compound $\mathbf{5 8}(301 \mathrm{mg}, 31 \%$ ) as a white semi-solid, and alcohol 22 (588 mg, 69\%) as a white solid. Ester 58: $\mathrm{R}_{f}=0.75\left(\mathrm{CH}_{2} \mathrm{Cl}_{2} / \mathrm{EtOAc}\right.$, 2/1); ${ }^{1} \mathrm{H}$ NMR (250 MHz, $\left.\mathrm{CDCl}_{3}\right) \delta 1.97$ (s, 3H), $4.12(\mathrm{dd}, J=14.0,8.5 \mathrm{~Hz}, 1 \mathrm{H}), 4.20(\mathrm{dd}, J=$ 11.0, $5.0 \mathrm{~Hz}, 1 \mathrm{H}), 4.29(\mathrm{dd}, J=14.0,2.0 \mathrm{~Hz}, 1 \mathrm{H}), 4.35(\mathrm{dd}, J=11.5,5.0 \mathrm{~Hz}, 1 \mathrm{H}), 4.41$ (dddd, $J$ $=8.5,5.0,5.0,2.0 \mathrm{~Hz}, 1 \mathrm{H}), 6.95(\mathrm{dd}, J=8.0,1.0 \mathrm{~Hz}, 1 \mathrm{H}), 7.05(\mathrm{dt}, J=8.0,1.0 \mathrm{~Hz}, 1 \mathrm{H}), 7.10-$ $7.26(\mathrm{~m}, 4 \mathrm{H}), 7.65-7.69(\mathrm{~m}, 1 \mathrm{H})$, and $8.44(\mathrm{dd}, J=8.0,1.5 \mathrm{~Hz}, 1 \mathrm{H}) ;{ }^{13} \mathrm{C}\left\{{ }^{1} \mathrm{H}\right\}$ NMR $(63 \mathrm{MHz}$, $\left.\mathrm{CDCl}_{3}\right) \delta 20.57,47.23,63.76,77.42,108.9,119.5(2 \times \mathrm{C}), 121.0,122.7,122.8,123.6,131.0$, 131.4, 135.9, 142.4, 149.8, 155.3, and 170.3; IR $\left(\mathrm{CHCl}_{3}\right) v_{\max } 1744,1475$, and $1445 \mathrm{~cm}^{-1}$; MS (ESI) $\mathrm{m} / z$ (rel intensity) $309\left(100 \%, \mathrm{MH}^{+}\right)$; HRMS calcd for $\mathrm{C}_{18} \mathrm{H}_{16} \mathrm{~N}_{2} \mathrm{NaO}_{3}\left(\mathrm{MNa}^{+}\right) 331.1059$, found 331.1064. Acetate $\mathbf{5 8}$ was optically resolved by CSP HPLC (Figure S5).

\section{(6,7-Dihydro-5-oxa-7a,12-diazadibenzo[a,e]azulen-6-yl)methanol (22)}

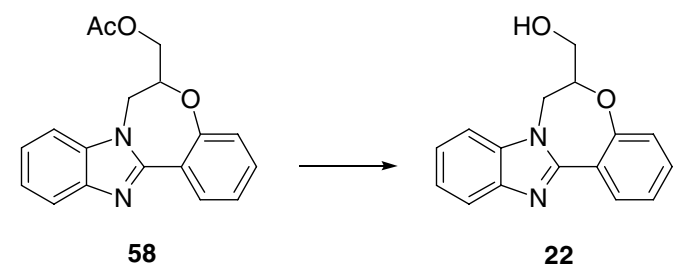

To a solution of ester $\mathbf{5 8}(128 \mathrm{mg}, 0.42 \mathrm{mmol})$ in THF ( $4 \mathrm{~mL})$ was added $1 \mathrm{M} \mathrm{LiOH} \mathrm{(1.3}$ $\mathrm{mL}, 1.3 \mathrm{mmol}$ ). The reaction mixture was stirred at $\mathrm{rt}$ for $17 \mathrm{~h}$, and partitioned between $\mathrm{CH}_{2} \mathrm{Cl}_{2}$ and water. The phases were separated, and the extraction was completed with additional portions of $\mathrm{CH}_{2} \mathrm{Cl}_{2}$. The combined organic extracts were dried $\left(\mathrm{MgSO}_{4}\right)$, and evaporated in vacuo to give a white solid. Purification by flash chromatography (silica gel, $\mathrm{CH}_{2} \mathrm{Cl}_{2} / \mathrm{EtOAc}, 1 / 1$ ) gave the title compound 22 (104 mg, 95\%), identical (TLC, ${ }^{1} \mathrm{H}$ NMR) with the product obtained from the cyclization of diol 20. 


\section{6,7-Dihydro-5-oxa-7a,12-diazadibenzo[a,e]azulen-6-ylmethyl toluene-4-sulfonate (59)}

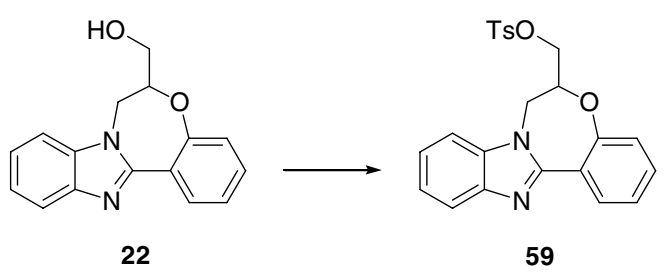

To an ice-cooled solution of alcohol 22 (266 $\mathrm{mg}, 1.00 \mathrm{mmol})$ in anhydrous pyridine (1 $\mathrm{mL})$ was added $\mathrm{TsCl}(238 \mathrm{mg}, 1.25 \mathrm{mmol})$. After $14 \mathrm{~h}$ at $0{ }^{\circ} \mathrm{C} \rightarrow \mathrm{rt}$, the reaction mixture was partitioned between $\mathrm{CH}_{2} \mathrm{Cl}_{2}$ and water, and neutralized with $1 \mathrm{M} \mathrm{HCl}$. The phases were separated and the extraction was completed with additional portions of $\mathrm{CH}_{2} \mathrm{Cl}_{2}$. The combined organic extracts were dried $\left(\mathrm{MgSO}_{4}\right)$ and evaporated in vacuo to give a white solid. Purification by flash chromatography (silica gel, $\mathrm{CH}_{2} \mathrm{Cl}_{2} \rightarrow \mathrm{CH}_{2} \mathrm{Cl}_{2} /$ EtOAc, 2/1) gave the title compound 59 (397 $\mathrm{mg}$, 95\%) as a white solid: $\mathrm{R}_{f}=0.25$ (petroleum ether/EtOAc, $2 / 1$ ); mp 165.5-166.5 ${ }^{\circ} \mathrm{C}$ (EtOAc/petroleum ether); ${ }^{1} \mathrm{H}$ NMR (400 MHz, $\left.\mathrm{CDCl}_{3}\right) \delta 2.46$ (s, 3H), 4.27 (dd, $\left.J=10.5,6.0 \mathrm{~Hz}, 1 \mathrm{H}\right), 4.31$ $(\mathrm{dd}, J=14.0,8.0 \mathrm{~Hz}, 1 \mathrm{H}), 4.41(\mathrm{dd}, J=10.5,5.0 \mathrm{~Hz}, 1 \mathrm{H}), 4.47(\mathrm{dd}, J=14.0,2.0 \mathrm{~Hz}, 1 \mathrm{H}), 4.61$ (dddd, $J=8.0,6.0,5.0,2.0 \mathrm{~Hz}, 1 \mathrm{H}), 6.99(\mathrm{~d}, J=8.0 \mathrm{~Hz}, 1 \mathrm{H}), 7.21(\mathrm{dt}, J=8.0,1.0 \mathrm{~Hz}, 1 \mathrm{H})$, 7.28-7.38 (m, 6H), 7.80-7.82 (m, $1 \mathrm{H}), 7.81(\mathrm{~d}, J=8.0 \mathrm{~Hz}, 2 \mathrm{H})$, and $8.52(\mathrm{dd}, J=8.0,1.5 \mathrm{~Hz}$, $1 \mathrm{H}) ;{ }^{13} \mathrm{C}\left\{{ }^{1} \mathrm{H}\right\} \mathrm{NMR}\left(101 \mathrm{MHz}, \mathrm{CDCl}_{3}\right) \delta 21.62,46.43,68.33,77.20,109.0,119.7(2 \times \mathrm{C}), 121.2$, $122.8,123.0,123.9,127.9,130.0,131.1,131.5,132.2$, 135.9, 142.7, 145.4, 149.9, and 154.6; IR $\left(\mathrm{CHCl}_{3}\right) v_{\max } 1611,1599,1577,1475,1446,1371$, and $1191 \mathrm{~cm}^{-1}$; MS (ESI) $\mathrm{m} / z$ (rel intensity) $421\left(100 \%, \mathrm{MH}^{+}\right)$; HRMS calcd for $\mathrm{C}_{23} \mathrm{H}_{20} \mathrm{~N}_{2} \mathrm{NaO}_{4} \mathrm{~S}\left(\mathrm{MNa}^{+}\right)$443.1041, found 443.1046; Anal. Calcd for $\mathrm{C}_{23} \mathrm{H}_{20} \mathrm{~N}_{2} \mathrm{O}_{4} \mathrm{~S}$ : C, 65.70; H, 4.79; N, 6.66; S, 7.63. Found: C, 65.71; H, 4.79; N, 6.65; S, 7.55.



To an ice-cooled solution of tosylate 59 (100 mg, $0.24 \mathrm{mmol})$ in THF (2 mL) was added $\mathrm{LiAlH}_{4}(76 \mathrm{mg}, 2.0 \mathrm{mmol})$. After $1.5 \mathrm{~h}$ at $0{ }^{\circ} \mathrm{C} \rightarrow \mathrm{rt}$, the reaction mixture was re-cooled in an ice bath, and quenched by a slow addition of water $(80 \mu \mathrm{L}), 15 \% \mathrm{NaOH}(240 \mu \mathrm{L})$, and water $(240$ $\mu \mathrm{L})$. The resulting thick suspension was diluted with $\mathrm{CH}_{2} \mathrm{Cl}_{2}$, and filtered through a thin pad of 
Celite $^{\circledR}$. The filtrate was evaporated in vacuo to give a clear oil. Purification by flash chromatography (silica gel, petroleum ether/EtOAc, 2/1) gave the title compound 23 (47 mg, $79 \%)$ as a white solid: $\mathrm{R}_{f}=0.25$ (petroleum ether/EtOAc, $\left.2 / 1\right) ;{ }^{1} \mathrm{H} \mathrm{NMR}\left(250 \mathrm{MHz}, \mathrm{CDCl}_{3}\right) 1.46$ (d, $J=6.5 \mathrm{~Hz}, 3 \mathrm{H}), 4.09$ (dd, $J=14.0,8.5 \mathrm{~Hz}, 1 \mathrm{H}), 4.27$ (dd, $J=14.0,2.0 \mathrm{~Hz}, 1 \mathrm{H}), 4.43$ (ddq, $J$ $=8.5,6.5,2.0 \mathrm{~Hz}, 1 \mathrm{H}), 6.96(\mathrm{dd}, J=8.0,1.0 \mathrm{~Hz}, 1 \mathrm{H}), 7.06(\mathrm{dt}, J=8.0,1.5 \mathrm{~Hz}, 1 \mathrm{H}), 7.12-7.26$ $(\mathrm{m}, 4 \mathrm{H}), 7.69-7.73(\mathrm{~m}, 1 \mathrm{H})$, and $8.53(\mathrm{dd}, J=8.0,1.5 \mathrm{~Hz}, 1 \mathrm{H}) ;{ }^{13} \mathrm{C}\left\{{ }^{1} \mathrm{H}\right\} \mathrm{NMR}\left(63 \mathrm{MHz}, \mathrm{CDCl}_{3}\right)$ $\delta 19.39,51.52,75.84,109.0,119.5,119.6,121.2,122.7$ ( $2 \times \mathrm{C}$ ?), 123.1, 131.2, 131.3, 136.2, 142.7, 150.3, and 155.9; IR $\left(\mathrm{CHCl}_{3}\right) v_{\max } 1609,1576,1476,1449,1385,1325$, and $1227 \mathrm{~cm}^{-1}$; MS (ESI) $\mathrm{m} / z$ (rel intensity) $251\left(100 \%, \mathrm{MH}^{+}\right)$; HRMS calcd for $\mathrm{C}_{16} \mathrm{H}_{15} \mathrm{~N}_{2} \mathrm{O}\left(\mathrm{MH}^{+}\right)$251.1184, found 251.1192 .

\section{(4-Chloro-11-methyl-6,7-dihydro-5-oxa-7a,12-diazadibenzo[a,e]azulen-6-yl)methanol (25) and cyclic ether (26)}
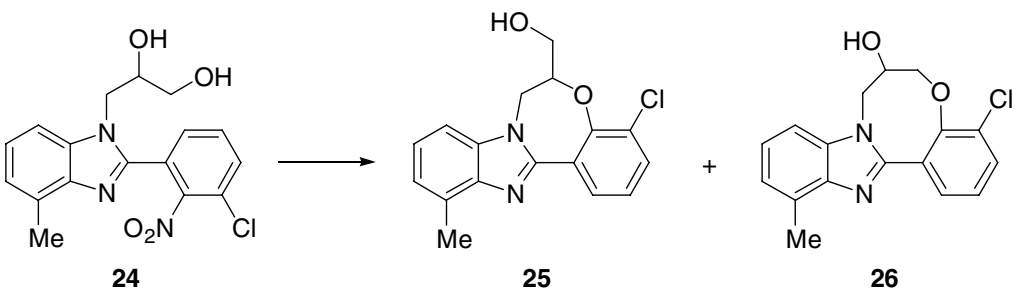

To a solution of diol $24(642 \mathrm{mg}, 1.78 \mathrm{mmol})$ in anhydrous DMF (4 mL) was added $\mathrm{NaH}$ (95\% w/w, $95 \mathrm{mg}, 3.9 \mathrm{mmol}$ ) to give a yellow solution. After $1 \mathrm{~h}$ at $\mathrm{rt}$, the reaction mixture was quenched with water, and diluted with $\mathrm{CH}_{2} \mathrm{Cl}_{2}$. The organic phase was washed repeatedly with water, dried $\left(\mathrm{MgSO}_{4}\right)$, and evaporated in vacuo to give a white solid. Purification by flash chromatography (silica gel, $\mathrm{CH}_{2} \mathrm{Cl}_{2} \rightarrow \mathrm{CH}_{2} \mathrm{Cl}_{2} / \mathrm{EtOAc}, 1 / 1$ ) gave the title compounds 25 (389 $\mathrm{mg}, 70 \%)$ and $26(45 \mathrm{mg}, 8 \%)$ as white solids. Benzimidazole 25: $\mathrm{R}_{f}=0.70\left(\mathrm{CH}_{2} \mathrm{Cl}_{2} / \mathrm{EtOAc}\right.$, 3/1); mp 186.5-188.0 ${ }^{\circ} \mathrm{C}$ (EtOAc/petroleum ether); ${ }^{1} \mathrm{H}$ NMR (400 MHz, $d_{6}$-DMSO) $\delta 2.61$ (s, $3 \mathrm{H}), 3.78^{9}$ (ddd, $\left.J=11.5,6.0,5.5 \mathrm{~Hz}, 1 \mathrm{H}\right), 3.88^{9}(\mathrm{ddd}, J=11.5,5.5,5.0 \mathrm{~Hz}, 1 \mathrm{H}), 4.44(\mathrm{dd}, J=$ 14.5, 8.0 Hz, 1H), 4.56 (dddd, $J=8.0,6.0,5.0,2.0 \mathrm{~Hz}, 1 \mathrm{H}$ ), 4.69 (dd, $J=14.5,2.0 \mathrm{~Hz}, 1 \mathrm{H}$ ), 5.23 [t, $J=5.5 \mathrm{~Hz}, 1 \mathrm{H}\left(\mathrm{D}_{2} \mathrm{O}\right.$ exchangeable)], $7.19(\mathrm{~d}, J=8.0 \mathrm{~Hz}, 1 \mathrm{H}), 7.20(\mathrm{t}, J=8.0 \mathrm{~Hz}, 1 \mathrm{H}), 7.23(\mathrm{t}$, $J=8.0 \mathrm{~Hz}, 1 \mathrm{H}), 7.41(\mathrm{~d}, J=8.0 \mathrm{~Hz}, 1 \mathrm{H}), 7.61(\mathrm{dd}, J=8.0,1.5 \mathrm{~Hz}, 1 \mathrm{H})$, and $8.44(\mathrm{dd}, J=8.0$, $1.5 \mathrm{~Hz}, 1 \mathrm{H}) ;{ }^{13} \mathrm{C}\left\{{ }^{1} \mathrm{H}\right\}$ NMR (101 MHz, $d_{6}$-DMSO) $\delta 16.33,46.68,61.52,82.11,107.7,122.3$, $122.6,122.7,123.7,125.5,128.7,129.5,131.3,135.9,141.5,148.0$, and 151.0; IR (KBr) $v_{\max }$

\footnotetext{
${ }^{9}$ In the presence of $\mathrm{D}_{2} \mathrm{O}$, the splitting pattern for the signals at 3.78 and $3.88 \mathrm{ppm}$ changed from 'ddd' to 'dd'.
} 
1472, 1459, and $1246 \mathrm{~cm}^{-1}$; MS (ESI) $\mathrm{m} / z$ (rel intensity) $337\left(30 \%, \mathrm{MNa}^{+}\right)$and 315 (100); HRMS calcd for $\mathrm{C}_{17} \mathrm{H}_{16} \mathrm{ClN}_{2} \mathrm{O}_{2}\left(\mathrm{MH}^{+}\right)$315.0900, found 315.0903; Anal. Calcd for $\mathrm{C}_{17} \mathrm{H}_{15} \mathrm{ClN}_{2} \mathrm{O}_{2}: \mathrm{C}, 64.87 ; \mathrm{H}, 4.80 ; \mathrm{Cl}, 11.26 ; \mathrm{N}, 8.90$. Found: C, 64.90; H, 4.83; Cl, 11.22; N, 8.84. Benzimidazole 26: $\mathrm{R}_{f}=0.60\left(\mathrm{CH}_{2} \mathrm{Cl}_{2} / \mathrm{EtOAc}, 3 / 1\right)$; $\mathrm{mp}>260{ }^{\circ} \mathrm{C}$ (EtOAc/petroleum ether); ${ }^{1} \mathrm{H}$ NMR (400 MHz, $d_{6}$-DMSO) $\delta 2.58(\mathrm{~s}, 3 \mathrm{H}), 3.98-4.04(\mathrm{~m}, 2 \mathrm{H}), 4.23(\mathrm{~d}, J=3.5 \mathrm{~Hz}, 1 \mathrm{H}), 4.29$ and $4.30(\mathrm{ABq}, J=14.5 \mathrm{~Hz}, 2 \mathrm{H}), 5.49\left[\left(\mathrm{~d}, J=4.0 \mathrm{~Hz}, 1 \mathrm{H}\left(\mathrm{D}_{2} \mathrm{O}\right.\right.\right.$ exchangeable $\left.)\right], 7.06(\mathrm{~d}, J=7.4$ $\mathrm{Hz}, 1 \mathrm{H}), 7.20$ (t, $J=7.5 \mathrm{~Hz}, 1 \mathrm{H}), 7.30$ (t, $J=8.0 \mathrm{~Hz}, 1 \mathrm{H}), 7.46(\mathrm{~d}, J=8.0 \mathrm{~Hz}, 1 \mathrm{H}), 7.71(\mathrm{dd}, J=$ $7.5,1.5 \mathrm{~Hz}, 1 \mathrm{H})$, and $7.72(\mathrm{dd}, J=8.0,1.5 \mathrm{~Hz}, 1 \mathrm{H}) ;{ }^{13} \mathrm{C}\left\{{ }^{1} \mathrm{H}\right\}$ NMR $\left(101 \mathrm{MHz}, d_{6}\right.$-DMSO) $\delta$ 16.35, 47.36, 65.56, 75.70, 108.3, 122.0, 122.5, 124.2, 124.7, 126.3, 128.6, 130.9, 132.1, 136.2, 141.8, 149.5, and 153.5; IR (KBr) $v_{\max } 1597,1471,1454,1432,1397,1265,1068$, and $994 \mathrm{~cm}^{-1}$; MS (ESI) $\mathrm{m} / z$ (rel intensity) $337\left(45 \%, \mathrm{MNa}^{+}\right.$) and 315 (100); HRMS calcd for $\mathrm{C}_{17} \mathrm{H}_{16} \mathrm{ClN}_{2} \mathrm{O}_{2}$ $\left(\mathrm{MH}^{+}\right)$315.0900, found 315.0890; Anal. Calcd for $\mathrm{C}_{17} \mathrm{H}_{15} \mathrm{ClN}_{2} \mathrm{O}_{2}$ : C, 64.87; $\mathrm{H}, 4.80 ; \mathrm{Cl}, 11.26$; N, 8.90. Found: C, 64.44; H, 4.82; Cl, 11.32; N, 8.80. Alcohols 25 and 26 were optically resolved by CSP HPLC (Figures S3 and S4, respectively).

\section{4-Chloro-11-methyl-6,7-dihydro-5-oxa-7a,12-diazadibenzo[a,e]azulen-6-ylmethyl toluene-4- sulfonate (60)

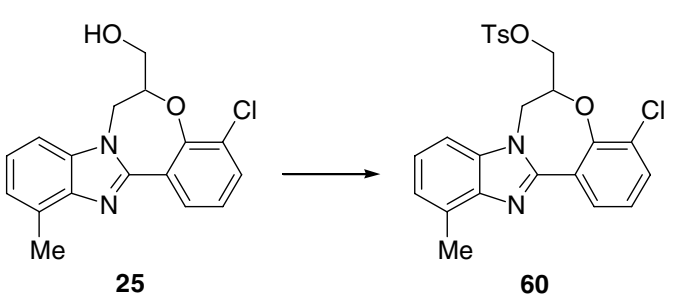

To an ice-cooled suspension of alcohol $25(314 \mathrm{mg}, 1.00 \mathrm{mmol})$ in anhydrous pyridine ( 2 $\mathrm{mL}$ ) was added $\mathrm{TsCl}(238 \mathrm{mg}, 1.25 \mathrm{mmol})$. After $17 \mathrm{~h}$ at $0{ }^{\circ} \mathrm{C} \rightarrow \mathrm{rt}$, the reaction mixture was partitioned between $\mathrm{CH}_{2} \mathrm{Cl}_{2}$ and water, and neutralized with $1 \mathrm{M} \mathrm{HCl}$. The phases were separated and the extraction was completed with additional portions of $\mathrm{CH}_{2} \mathrm{Cl}_{2}$. The combined organic extracts were dried $\left(\mathrm{MgSO}_{4}\right)$ and evaporated in vacuo to give a white solid. Purification by flash chromatography (silica gel, $\mathrm{CH}_{2} \mathrm{Cl}_{2} \rightarrow \mathrm{CH}_{2} \mathrm{Cl}_{2} / \mathrm{EtOAc}$, 5/1) gave the title compound $\mathbf{6 0}$ (441 mg, 94\%) as a white solid: $\mathrm{R}_{f}=0.50$ (petroleum ether/EtOAc, 2/1); mp 168.0-169.0 ${ }^{\circ} \mathrm{C}$ (EtOAc/petroleum ether); ${ }^{1} \mathrm{H}$ NMR $\left(250 \mathrm{MHz}, \mathrm{CDCl}_{3}\right) \delta 2.32(\mathrm{~s}, 3 \mathrm{H}), 2.58(\mathrm{~s}, 3 \mathrm{H}), 4.12-4.36(\mathrm{~m}$, 4H), 4.52-4.60 (m, 1H), 6.97-7.14 (m, 4H), $7.22(\mathrm{~d}, J=8.0 \mathrm{~Hz}, 1 \mathrm{H}), 7.31$ (dd, $J=8.0,1.5 \mathrm{~Hz}$, $1 \mathrm{H}), 7.69(\mathrm{~d}, J=8.0 \mathrm{~Hz}, 1 \mathrm{H})$, and $8.25(\mathrm{dd}, J=8.0,1.5 \mathrm{~Hz}, 1 \mathrm{H}) ;{ }^{13} \mathrm{C}\left\{{ }^{1} \mathrm{H}\right\} \mathrm{NMR}(63 \mathrm{MHz}$, 
$\left.\mathrm{CDCl}_{3}\right) \delta 17.05,22.09,46.41,68.60,79.05,107.1,123.6,123.7,123.8,125.0,127.1,128.5$, 130.1, 130.5, 130.7, 132.1, 132.6, 136.1, 142.6, 145.9, 148.8, and 150.6; IR $\left(\mathrm{CHCl}_{3}\right) v_{\max } 1469$, $1450,1432,1373,1228$, and $1190 \mathrm{~cm}^{-1}$; MS (ESI) $\mathrm{m} / z$ (rel intensity) $491\left(60 \%, \mathrm{MNa}^{+}\right)$and 469 (100); HRMS calcd for $\mathrm{C}_{24} \mathrm{H}_{21} \mathrm{ClN}_{2} \mathrm{NaO}_{4} \mathrm{~S}\left(\mathrm{MNa}^{+}\right)$391.0808, found 391.0803; Anal. Calcd for $\mathrm{C}_{24} \mathrm{H}_{21} \mathrm{ClN}_{2} \mathrm{O}_{4} \mathrm{~S}: \mathrm{C}, 61.47 ; \mathrm{H}, 4.51 ; \mathrm{Cl}, 7.56 ; \mathrm{N}, 5.97 ; \mathrm{S}, 6.84$. Found: C, 61.61; H, 4.49; Cl, 7.69; N, 5.93; S, 6.78.

\section{8-(1-Chlorovinyl)-1,6,9-trimethyl-5,6-dihydro-7-oxa-4b,10-diazabenzo[a]azulene (27)}

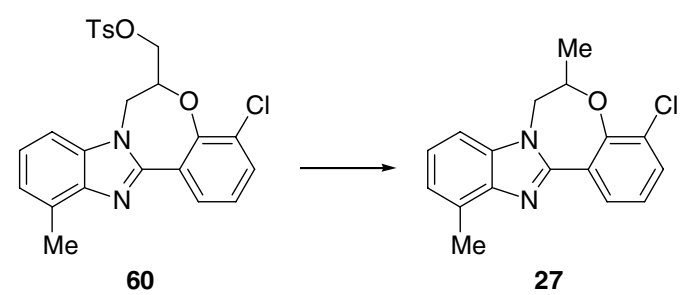

To an ice-cooled solution of tosylate $\mathbf{6 0}(100 \mathrm{mg}, 0.21 \mathrm{mmol})$ in THF (2 mL) was added $\mathrm{LiAlH}_{4}(40 \mathrm{mg}, 1.1 \mathrm{mmol})$. After $3 \mathrm{~h}$ at $0{ }^{\circ} \mathrm{C} \rightarrow \mathrm{rt}$, the reaction mixture was re-cooled to $0{ }^{\circ} \mathrm{C}$, and quenched by a slow addition of water $(40 \mu \mathrm{L}), 15 \% \mathrm{NaOH}(120 \mu \mathrm{L})$, and water $(40 \mu \mathrm{L})$. The resulting thick suspension was diluted with $\mathrm{CH}_{2} \mathrm{Cl}_{2}$, and filtered through a thin pad of Celite ${ }^{\circledR}$. The filtrate was evaporated in vacuo to give a clear oil. Purification by flash chromatography ( silica gel, petroleum ether/ $\left.\mathrm{CH}_{2} \mathrm{Cl}_{2}, 1 / 1\right)$ gave the title compound $27(59 \mathrm{mg}, 93 \%$ ) as a white solid: ${ }^{1} \mathrm{H}$ NMR (400 MHz, $\left.\mathrm{CDCl}_{3}\right) 1.67(\mathrm{dt}, J=6.5,1.5 \mathrm{~Hz}, 3 \mathrm{H}), 2.75(\mathrm{~s}, 3 \mathrm{H}), 4.25$ (ddt, $J=$ 14.0, 8.5, 1.5 Hz, 1H), $4.38(\sim \mathrm{d}, J=14.0 \mathrm{~Hz}, 1 \mathrm{H}), 4.66(\mathrm{~m}, 1 \mathrm{H}), 7.11-7.28(\mathrm{~m}, 4 \mathrm{H}), 7.48$ ( dd, $J$ $=8.0,1.5 \mathrm{~Hz}, 1 \mathrm{H})$, and $8.55(\mathrm{dd}, J=8.0,1.5 \mathrm{~Hz}, 1 \mathrm{H}) ;{ }^{13} \mathrm{C}\left\{{ }^{1} \mathrm{H}\right\} \mathrm{NMR}\left(63 \mathrm{MHz}, \mathrm{CDCl}_{3}\right) \delta 16.61$, 19.36, 51.12, 77.61, 106.5, 122.8, 123.0, 123.1, 123.7, 126.5, 129.7, 130.1, 131.4, 135.8, 142.0, 148.6, and 151.4; IR $\left(\mathrm{CHCl}_{3}\right) v_{\max }$ 1470, 1452, 1431, 1422, and $1385 \mathrm{~cm}^{-1}$; MS (ESI) $\mathrm{m} / z$ (rel intensity) $321\left(55 \%, \mathrm{MNa}^{+}\right)$and 299 (100); HRMS calcd for $\mathrm{C}_{17} \mathrm{H}_{16} \mathrm{ClN}_{2} \mathrm{O}\left(\mathrm{MH}^{+}\right)$299.0951, found 299.0930.

(4-Chloro-6,7-dihydro-5-oxa-7a,12-diazadibenzo[a,e]azulen-6-yl)methanol (61)

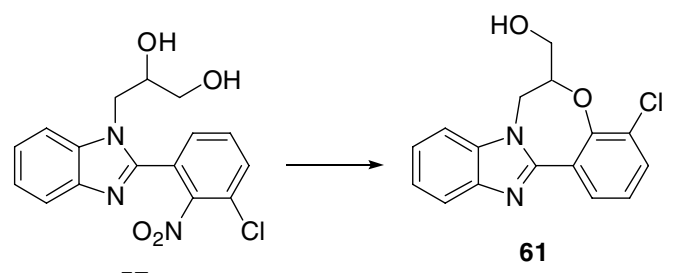

57 
To a solution of diol $57(100 \mathrm{mg}, 0.29 \mathrm{mmol})$ in anhydrous DMF $(2 \mathrm{~mL})$ was added $\mathrm{NaH}$ (60\% w/w, $25.3 \mathrm{mg}, 0.63 \mathrm{mmol})$. After $21 \mathrm{~h}$ at $\mathrm{rt}$, the reaction mixture was quenched with water, and diluted with EtOAc. The organic phase was washed repeatedly with water, dried $\left(\mathrm{MgSO}_{4}\right)$, and evaporated in vacuo to give an off-white solid. Purification by flash chromatography (silica gel, $\left.\mathrm{CH}_{2} \mathrm{Cl}_{2} \rightarrow \mathrm{CH}_{2} \mathrm{Cl}_{2} / \mathrm{EtOAc}, 1 / 1\right)$ gave the title compound $\mathbf{6 1}(70 \mathrm{mg}, 81 \%)$ as a white solid: $\mathrm{R}_{f}=0.75$ (EtOAc); ${ }^{1} \mathrm{H}$ NMR (400 MHz, $d_{6}$-DMSO) $\delta 3.83(\mathrm{dd}, J=11.0,5.5 \mathrm{~Hz}, 1 \mathrm{H}), 3.92(\mathrm{dd}, J$ $=11.0,5.0 \mathrm{~Hz}, 1 \mathrm{H}), 4.52(\mathrm{dd}, J=14.5,8.5 \mathrm{~Hz}, 1 \mathrm{H}), 4.64(\mathrm{dddd}, J=8.5,5.5,5.0,2.0 \mathrm{~Hz}, 1 \mathrm{H})$, 4.77 (dd, $J=14.5,2.0 \mathrm{~Hz}, 1 \mathrm{H}), 5.27$ (br s, $1 \mathrm{H}), 7.28$ (t, $J=8.0 \mathrm{~Hz}, 1 \mathrm{H}), 7.33$ (dt, $J=7.0,1.0 \mathrm{~Hz}$, $1 \mathrm{H}), 7.37(\mathrm{dt}, J=7.0,1.0 \mathrm{~Hz}, 1 \mathrm{H}), 7.63-7.69(\mathrm{~m}, 2 \mathrm{H}), 7.77(\sim \mathrm{d}, J=7.0 \mathrm{~Hz}, 1 \mathrm{H})$, and $8.46(\mathrm{dd}, J$ $=8.0,1.5 \mathrm{~Hz}, 1 \mathrm{H}) ;{ }^{13} \mathrm{C}\left\{{ }^{1} \mathrm{H}\right\} \mathrm{NMR}\left(101 \mathrm{MHz}, d_{6}\right.$-DMSO) $\delta 46.63,61.53,82.10,110.4,119.1$, $122.1,122.5,122.8,123.7,125.6,129.5,131.5,136.2,142.1,148.9$, and 151.2; IR (KBr) $v_{\max }$ 1474, 1455, 1427, 1259, 1247, 1099, and $1058 \mathrm{~cm}^{-1}$; MS (ESI) $\mathrm{m} / z$ (rel intensity) 301 (100\%, $\mathrm{MH}^{+}$); HRMS calcd for $\mathrm{C}_{16} \mathrm{H}_{14} \mathrm{ClN}_{2} \mathrm{O}_{2}\left(\mathrm{MH}^{+}\right)$301.0744, found 301.0728. Alcohol 61 was optically resolved by CSP HPLC (Figure S6).

\subsection{Intermolecular $S_{N} A r$ Reactions}

\section{1-Methyl-2-(2-nitrophenyl)-1H-benzimidazole (31)}

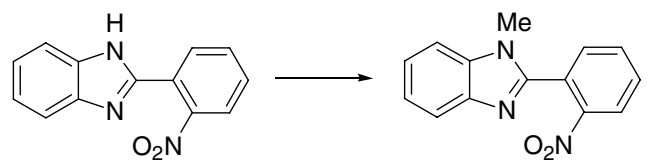

50

31

To an ice-cooled suspension of benzimidazole $\mathbf{5 0}^{6}(2.00 \mathrm{~g}, 8.37 \mathrm{mmol})$ in THF $(15 \mathrm{~mL})$ was added $\mathrm{NaH}(60 \% \mathrm{w} / \mathrm{w}, 368 \mathrm{mg}, 9.2 \mathrm{mmol})$ portionwise over $5 \mathrm{~min}$. After $10 \mathrm{~min}$ at $0{ }^{\circ} \mathrm{C}$, the resulting red solution was treated with $\mathrm{MeI}(1.3 \mathrm{~mL}, 21 \mathrm{mmol})$, and stirred at $0{ }^{\circ} \mathrm{C} \rightarrow \mathrm{rt}$ for $16 \mathrm{~h}$. The reaction mixture was quenched with water, evaporated in vacuo, and partitioned between $\mathrm{CH}_{2} \mathrm{Cl}_{2}$ and water. The phases were separated, and the extraction was completed with additional portions of $\mathrm{CH}_{2} \mathrm{Cl}_{2}$. The combined organic extracts were dried $\left(\mathrm{MgSO}_{4}\right)$, and evaporated in vacuo. Purification by flash chromatography (silica gel, EtOAc/petroleum ether, 1/1) gave the title compound $31(1.52 \mathrm{~g}, 72 \%)$ as a yellow solid: $\mathrm{R}_{f}=0.60$ (EtOAc); $\mathrm{mp} 132.5-133.5{ }^{\circ} \mathrm{C}$ (EtOAc/petroleum ether) $\left(\mathrm{Lit}^{10}{ }^{10} 135-137{ }^{\circ} \mathrm{C}\right) ;{ }^{1} \mathrm{H}$ NMR $\left(400 \mathrm{MHz}, \mathrm{CDCl}_{3}\right) \delta 3.60$ (s, 3H), 7.32

\footnotetext{
${ }^{10}$ Hawkins, D.; Lindley, J. M.; McRobbie, I. M.; Meth-Cohn, O. J. Chem. Soc., Perkin Trans. 1, 1980, 2387-2391.
} 
$(\mathrm{dt}, J=7.5,1.5 \mathrm{~Hz}, 1 \mathrm{H}), 7.36(\mathrm{dt}, J=7.5,1.5 \mathrm{~Hz}, 1 \mathrm{H}), 7.41(\mathrm{dd}, J=7.0,1.5 \mathrm{~Hz}, 1 \mathrm{H}), 7.64(\mathrm{dd}, J$ $=8.0,1.5 \mathrm{~Hz}, 1 \mathrm{H}), 7.68(\mathrm{dt}, J=8.0,1.5 \mathrm{~Hz}, 1 \mathrm{H}), 7.75(\mathrm{dt}, J=7.5,1.0 \mathrm{~Hz}, 1 \mathrm{H}), 7.81(\mathrm{dd}, J=7.0$, $1.5 \mathrm{~Hz}, 1 \mathrm{H})$, and $8.18(\mathrm{dd}, J=8.0,1.0 \mathrm{~Hz}, 1 \mathrm{H}) ;{ }^{13} \mathrm{C}\left\{{ }^{1} \mathrm{H}\right\} \mathrm{NMR}\left(101 \mathrm{MHz}, \mathrm{CDCl}_{3}\right) \delta 30.39$, 109.5, 119.8, 122.3, 123.0, 124.6, 125.8, 130.9, 132.6, 133.4, 135.5, 142.7, 148.5, and 149.6; IR $\left(\mathrm{CHCl}_{3}\right) v_{\max } 1534,1462,1438,1394$, and $1348 \mathrm{~cm}^{-1}$; MS (ESI) $\mathrm{m} / z$ (rel intensity) 254 (100\%, $\mathrm{MH}^{+}$) and 207 (25); HRMS calcd for $\mathrm{C}_{14} \mathrm{H}_{12} \mathrm{~N}_{3} \mathrm{O}_{2}\left(\mathrm{MH}^{+}\right)$254.0909, found 254.0906; Anal. Calcd for $\mathrm{C}_{14} \mathrm{H}_{11} \mathrm{~N}_{3} \mathrm{O}_{2}$ : C, 66.40; H, 4.38; N, 16.59. Found: C, 66.69; H, 4.40; N, 16.72 .

\section{2-(2-Methoxyphenyl)-1-methyl-1H-benzimidazole (32) and}

\section{2-(2-methoxyphenyl)-1,3-dimethyl-3H-benzimidazol-1-ium iodide (62)}

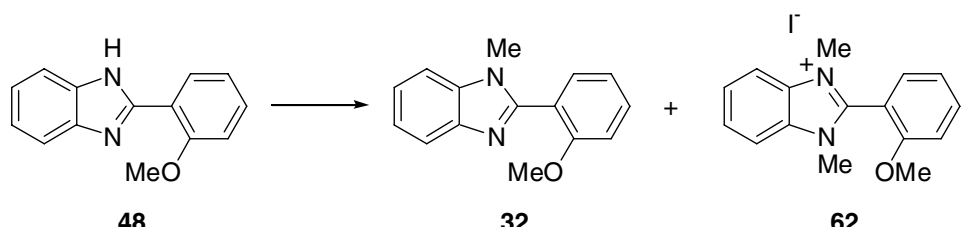

To a solution of benzimidazole $48(1.00 \mathrm{~g}, 4.20 \mathrm{mmol})$ in THF $(20 \mathrm{~mL})$ was added $\mathrm{NaH}$ $(60 \% \mathrm{w} / \mathrm{w}, 185 \mathrm{mg}, 4.6 \mathrm{mmol})$ portionwise at $0{ }^{\circ} \mathrm{C}$. After $15 \mathrm{~min}$ at $\mathrm{rt}$, the resulting red solution was treated with MeI $(314 \mu \mathrm{L}, 5.0 \mathrm{mmol})$, and stirred at $\mathrm{rt}$ for $18 \mathrm{~h}$. The reaction mixture was quenched with water, evaporated in vacuo, and partitioned between $\mathrm{CH}_{2} \mathrm{Cl}_{2}$ and water. The phases were separated, and the extraction was completed with additional portions of $\mathrm{CH}_{2} \mathrm{Cl}_{2}$. The combined organic extracts were dried $\left(\mathrm{MgSO}_{4}\right)$, and evaporated in vacuo to give a white solid. Purification by flash chromatography (silica gel, $\mathrm{CH}_{2} \mathrm{Cl}_{2} \rightarrow \mathrm{CH}_{2} \mathrm{Cl}_{2} / \mathrm{EtOAc}$, 20/1) gave the title compound 32 (600 mg, 57\%) as a clear oil that solidified on standing: $\mathrm{R}_{f}=0.65$ (EtOAc); ${ }^{1} \mathrm{H}$ NMR (400 MHz, $\left.\mathrm{CDCl}_{3}\right) \delta 3.61(\mathrm{~s}, 3 \mathrm{H}), 3.76(\mathrm{~s}, 3 \mathrm{H}), 6.99(\mathrm{~d}, J=8.5 \mathrm{~Hz}, 1 \mathrm{H}), 7.07$ (dt, $J=7.5$, $0.5 \mathrm{~Hz}, 1 \mathrm{H}), 7.22-7.30(\mathrm{~m}, 2 \mathrm{H}), 7.32-7.38(\mathrm{~m}, 1 \mathrm{H}), 7.46(\mathrm{dt}, J=8.5,1.5 \mathrm{~Hz}, 1 \mathrm{H}), 7.56(\mathrm{dd}, J=$ 7.5, $1.5 \mathrm{~Hz}, 1 \mathrm{H})$, and 7.77-7.82 $(\mathrm{m}, 1 \mathrm{H}) ;{ }^{13} \mathrm{C}\left\{{ }^{1} \mathrm{H}\right\} \mathrm{NMR}\left(101 \mathrm{MHz}, \mathrm{CDCl}_{3}\right) \delta$ 31.28, 55.97, $109.9,111.5,120.1,120.2,121.4,122.3,122.8,132.0,132.8,136.5,143.6,152.6$, and 158.0; IR $\left(\mathrm{CHCl}_{3}\right) v_{\max } 1609,1477,1463,1439,1388$, and $1254 \mathrm{~cm}^{-1}$; MS (ESI) $\mathrm{m} / z$ (rel intensity) 239 $\left(100 \%, \mathrm{MH}^{+}\right)$; HRMS calcd for $\mathrm{C}_{15} \mathrm{H}_{15} \mathrm{~N}_{2} \mathrm{O}\left(\mathrm{MH}^{+}\right)$239.1184, found 239.1161 .

When an analogous reaction was performed with an excess of MeI (2.5 equiv.), only a small amount $(\sim 10 \%)$ of benzimidazole 32 was isolated. Instead, the benzimidazolium salt $\mathbf{6 2}$ was obtained as the major product $(77 \%)$. Salt 62: a white solid: $\mathrm{mp}>260{ }^{\circ} \mathrm{C}$ (EtOAc); ${ }^{1} \mathrm{H}$ NMR $\left(250 \mathrm{MHz}, d_{4}-\mathrm{MeOH}\right) \delta 3.81(\mathrm{~s}, 6 \mathrm{H}), 3.84(\mathrm{~s}, 3 \mathrm{H}), 7.23(\mathrm{dt}, J=7.5,1.5 \mathrm{~Hz}, 1 \mathrm{H}), 7.33(\mathrm{~d}, J=8.5$ 
$\mathrm{Hz}, 1 \mathrm{H}), 7.60-7.69(\mathrm{~m}, 3 \mathrm{H}), 7.75(\mathrm{ddd}, J=8.5,7.5,1.5 \mathrm{~Hz}, 1 \mathrm{H})$, and 7.86-7.92 $(\mathrm{m}, 2 \mathrm{H}) ;{ }^{13} \mathrm{C}\left\{{ }^{1} \mathrm{H}\right\}$ NMR $\left(101 \mathrm{MHz}, d_{4}-\mathrm{MeOH}\right) \delta 33.32,56.98,110.4,113.8,114.2,122.7,128.2,133.1,133.5$, 136.9, 150.2, and 159.6; IR (KBr) $v_{\max } 1603,1582,1516,1487,1470,1446,1435$, and $1259 \mathrm{~cm}^{-}$

${ }^{1}$; MS (ESI) $m / z$ (rel intensity) 253 (100\%, M-I-), 237 (80), and 221 (7); HRMS calcd for $\mathrm{C}_{16} \mathrm{H}_{17} \mathrm{~N}_{2} \mathrm{O}\left(\mathrm{M}-\mathrm{I}^{-}\right)$253.1341, found 253.1325.

\section{2-(2-Methoxyphenyl)-1-methyl-1H-benzimidazole (32)}

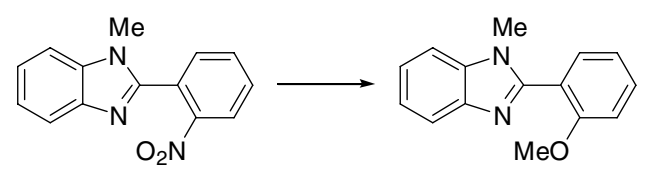

31

32

To a solution of benzimidazole 31 (100 mg, $0.40 \mathrm{mmol})$ in anhydrous DMF ( $2 \mathrm{~mL}$ ) was added $\mathrm{MeONa}$ (213 mg, $3.95 \mathrm{mmol}$ ). After $20 \mathrm{~h}$ at $100{ }^{\circ} \mathrm{C}$, the reaction mixture was cooled to $\mathrm{rt}$, and partitioned between EtOAc and water. The organic layer was washed repeatedly with water, dried $\left(\mathrm{MgSO}_{4}\right)$, and evaporated in vacuo to give a yellow solid. Purification by flash chromatography (silica gel, $\mathrm{CH}_{2} \mathrm{Cl}_{2} \rightarrow \mathrm{CH}_{2} \mathrm{Cl}_{2}$ /EtOAc, 3/1) gave an inseparable $~ 1.9: 1$ mixture $(87 \mathrm{mg}$ ) of the starting material $\mathbf{3 1}$ and the title compound $\mathbf{3 2}$ as a yellow oil. When an analogous reaction was carried out at $\mathrm{rt}$ for $25 \mathrm{~h}$, it gave a $\sim 3.3: 1$ mixture $(97 \mathrm{mg}$ ) of the starting material 31 and the title compound $\mathbf{3 2}$.

\section{1-Methyl-2-(4-nitrophenyl)-1H-benzimidazole (33)}

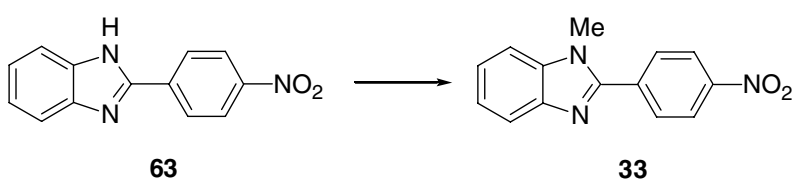

To a suspension of benzimidazole $\mathbf{6 3}^{11}(2.00 \mathrm{~g}, 8.37 \mathrm{mmol})$ in THF $(15 \mathrm{~mL})$ was added $\mathrm{NaH}(60 \% \mathrm{w} / \mathrm{w}, 368 \mathrm{mg}, 9.2 \mathrm{mmol})$ portionwise at $0{ }^{\circ} \mathrm{C}$. After $15 \mathrm{~min}$ at $\mathrm{rt}$, the resulting red solution was treated with $\mathrm{MeI}(1.3 \mathrm{~mL}, 21 \mathrm{mmol})$, and stirred at $\mathrm{rt}$ for $19 \mathrm{~h}$. The reaction mixture was quenched with water, evaporated in vacuo, and partitioned between $\mathrm{CH}_{2} \mathrm{Cl}_{2}$ and water. The phases were separated, and the extraction was completed with additional portions of $\mathrm{CH}_{2} \mathrm{Cl}_{2}$. The combined organic extracts were dried $\left(\mathrm{MgSO}_{4}\right)$, and evaporated in vacuo. Purification by flash chromatography (silica gel, $\mathrm{CH}_{2} \mathrm{Cl}_{2} \rightarrow \mathrm{CH}_{2} \mathrm{Cl}_{2} / \mathrm{EtOAc}$, 20/1) gave the title compound $\mathbf{3 3}$ (1.47 g, $69 \%)$ as a yellow solid: $\mathrm{R}_{f}=0.75\left(\mathrm{EtOAc} / \mathrm{CH}_{2} \mathrm{Cl}_{2}, 1 / 1\right) ; \mathrm{mp} 208.0-209.0{ }^{\circ} \mathrm{C}(\mathrm{EtOAc} /$ petroleum

\footnotetext{
${ }^{11}$ Prabhakar Reddy, V.; Prasunamba, P. L.; Reddy, P. S. N.; Ratnam, C. V. Ind. J. Chem. Sect. B 1983, 22, 917-918.
} 
ether) (Lit. $\left.{ }^{12} 211-213{ }^{\circ} \mathrm{C}\right) ;{ }^{1} \mathrm{H} \mathrm{NMR}\left(400 \mathrm{MHz}, \mathrm{CDCl}_{3}\right) \delta 3.91(\mathrm{~s}, 3 \mathrm{H}), 7.34(\mathrm{dt}, J=7.0,1.0 \mathrm{~Hz}$, $1 \mathrm{H}), 7.37(\sim \mathrm{t}, J=7.0 \mathrm{~Hz}, 1 \mathrm{H}), 7.42(\mathrm{dd}, J=7.5,1.5 \mathrm{~Hz}, 1 \mathrm{H}), 7.83(\mathrm{dd}, J=7.5,1.5 \mathrm{~Hz}, 1 \mathrm{H}), 7.98$ $(\mathrm{d}, J=8.5 \mathrm{~Hz}, 2 \mathrm{H})$, and $8.37(\mathrm{~d}, J=8.5 \mathrm{~Hz}, 2 \mathrm{H}) ;{ }^{13} \mathrm{C}\left\{{ }^{1} \mathrm{H}\right\}$ NMR $\left(101 \mathrm{MHz}, \mathrm{CDCl}_{3}\right) \delta 31.92$, $109.9,120.3,123.1,123.8,123.9,130.3,136.3,136.7,142.8,148.4$, and 151.0; IR $\left(\mathrm{CHCl}_{3}\right) v_{\max }$ 1604, 1527, and $1350 \mathrm{~cm}^{-1}$; MS (ESI) $\mathrm{m} / z$ (rel intensity) $254\left(100 \%, \mathrm{MH}^{+}\right.$) and $208(20)$; HRS calcd for $\mathrm{C}_{14} \mathrm{H}_{12} \mathrm{~N}_{3} \mathrm{O}_{2}\left(\mathrm{MH}^{+}\right)$254.0929, found 254.0936; Anal. Calcd for $\mathrm{C}_{14} \mathrm{H}_{11} \mathrm{~N}_{3} \mathrm{O}_{2}$ : C, 66.40; H, 4.38; N, 16.59. Found: C, 66.49; H, 4.41; N, 16.66.

\section{2-(4-Methoxyphenyl)-1-methyl-1H-benzimidazole (34)}

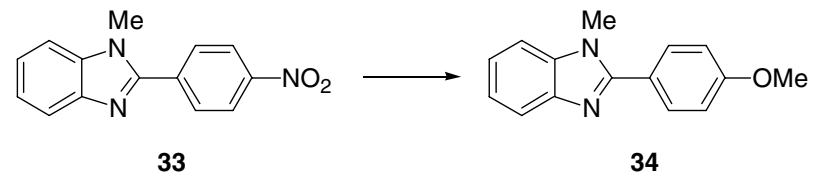

To a suspension of benzimidazole $33(100 \mathrm{mg}, 0.40 \mathrm{mmol})$ in anhydrous DMF (2 mL) was added MeONa (213 mg, $3.95 \mathrm{mmol})$. After $18 \mathrm{~h}$ at $100{ }^{\circ} \mathrm{C}$, the reaction mixture was cooled to rt, and partitioned between EtOAc and water. The organic layer was washed repeatedly with water, dried $\left(\mathrm{MgSO}_{4}\right)$, and evaporated in vacuo to give a yellow solid. Purification by flash chromatography (silica gel, $\mathrm{CH}_{2} \mathrm{Cl}_{2} \rightarrow \mathrm{CH}_{2} \mathrm{Cl}_{2}$ /EtOAc, 20/1) gave the recovered starting material 33 (42 $\mathrm{mg}, 42 \%)$, and the title compound 34 (53 mg, 56\%) as a white solid. When an analogous reaction was carried out at $\mathrm{rt}$ for $24 \mathrm{~h}$, it gave the recovered starting material 33 (73 $\mathrm{mg}, 73 \%$ ) and the title compound 34 (23 mg, 24\%). Benzimidazole 34: $\mathrm{R}_{f}=0.25$ (EtOAc/petroleum ether, 1/1); ${ }^{1} \mathrm{H}$ NMR (400 MHz, $\left.\mathrm{CDCl}_{3}\right) \delta 3.84(\mathrm{~s}, 3 \mathrm{H}), 3.89$ (s, 3H), 7.06 ( dt, $\left.J=9.0,2.5 \mathrm{~Hz}, 2 \mathrm{H}\right)$, 7.28-7.39 (m, 3H), $7.73(\sim \mathrm{dt}, J=9.0,2.5 \mathrm{~Hz}, 2 \mathrm{H})$, and 7.82-7.86 (m, 1H); ${ }^{13} \mathrm{C}\left\{{ }^{1} \mathrm{H}\right\} \mathrm{NMR}(63$ $\left.\mathrm{MHz}, \mathrm{CDCl}_{3}\right) \delta 31.58,55.28,109.4,114.0,119.4,122.2,122.3,122.4,130.7,136.4,142.7$, 153.6, and 160.7; IR $\left(\mathrm{CHCl}_{3}\right) v_{\max } 1614,1485,1462,1436,1384,1253$, and $1176 \mathrm{~cm}^{-1}$; MS (ESI) $m / z$ (rel intensity) $239\left(100 \%, \mathrm{MH}^{+}\right)$; HRMS calcd for $\mathrm{C}_{15} \mathrm{H}_{15} \mathrm{~N}_{2} \mathrm{O}\left(\mathrm{MH}^{+}\right) 239.1184$, found 239.1171 .

\footnotetext{
${ }^{12}$ El'tsov, A. V.; Muravich-Aleksandr, Kh. L. J. Org. Chem. USSR (Engl.) 1965, 1321-1327.
} 


\section{Selected CSP HPLC Profiles}

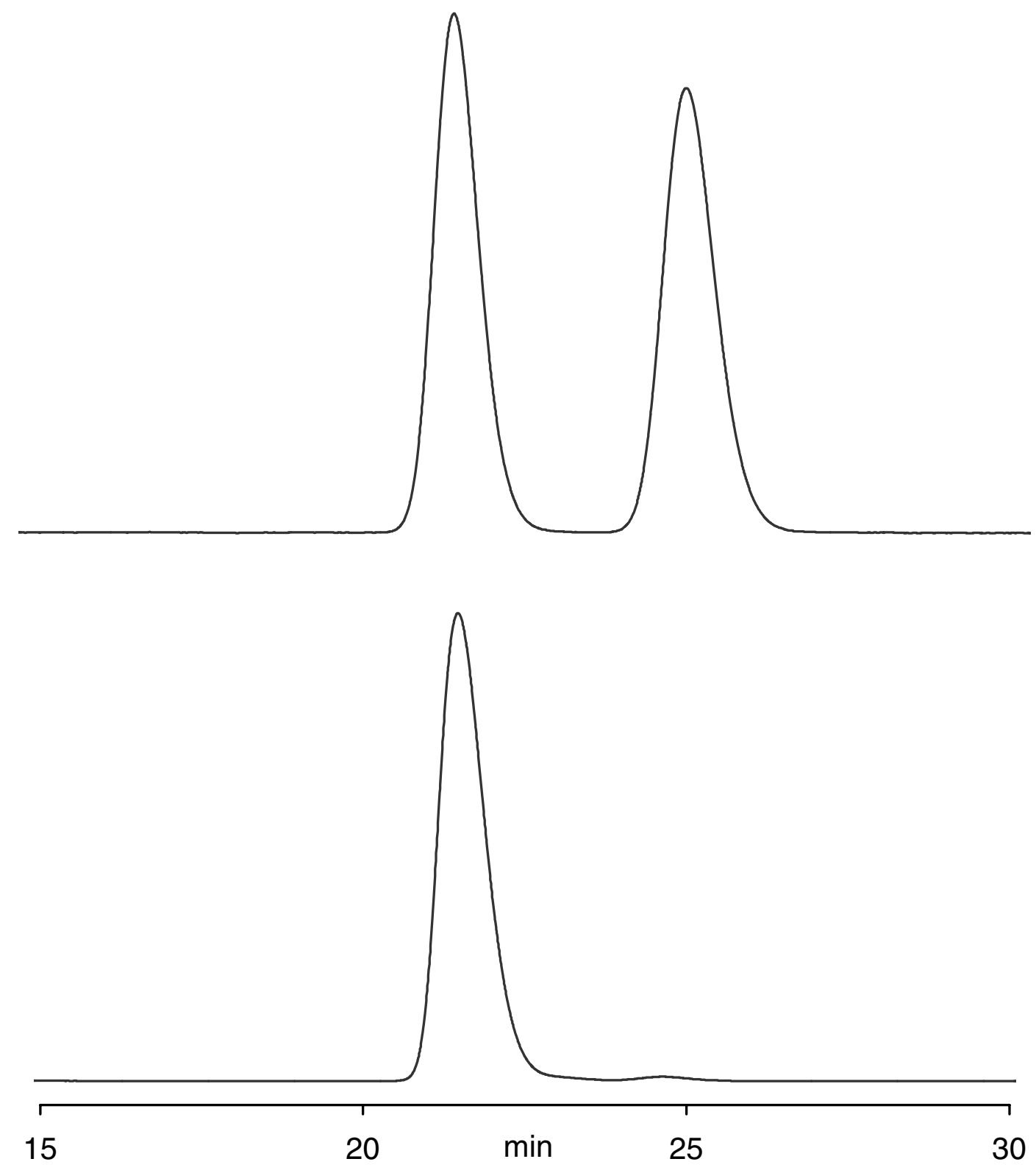

Figure S1. Optical resolution of the cyclic ether 3 by CSP HPLC (Chiralcel OD column, $4.6 \mathrm{~mm}$ $\times 25 \mathrm{~cm}$; 2-propanol/hexanes, 10/90; $1 \mathrm{~mL} \mathrm{~min}^{-1} ; 40{ }^{\circ} \mathrm{C} ; 254 \mathrm{~nm}$ ). Top: the racemate; bottom: an enantiomerically enriched sample (ee $=98.0 \%$ ) obtained by the intermolecular nitro-group displacement in alcohol $(+)-\mathbf{1}(\mathrm{ee}=98.0 \%)$. 

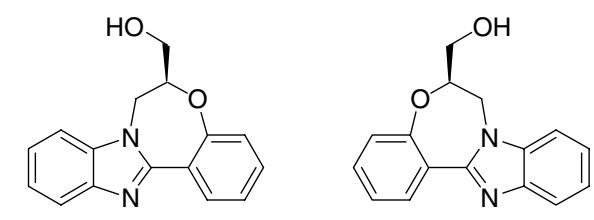

22

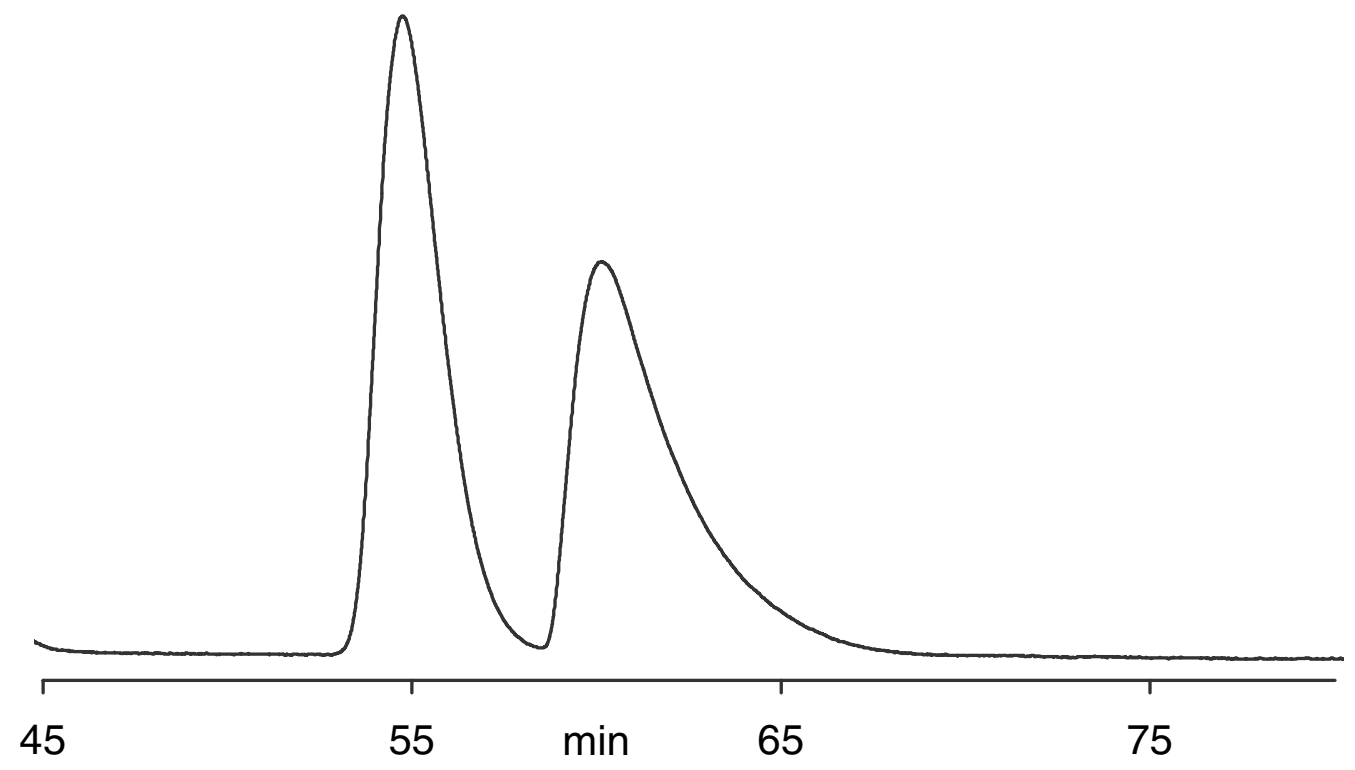

Figure S2. Optical resolution of alcohol 22 by CSP HPLC (Chiralcel OD column, $4.6 \mathrm{~mm} \times 25$ cm; 2-propanol/hexanes, 15/85; $1 \mathrm{~mL} \mathrm{~min}^{-1} ; 40{ }^{\circ} \mathrm{C} ; 254 \mathrm{~nm}$ ). 

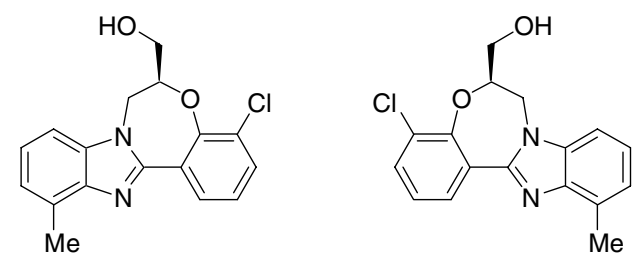

25

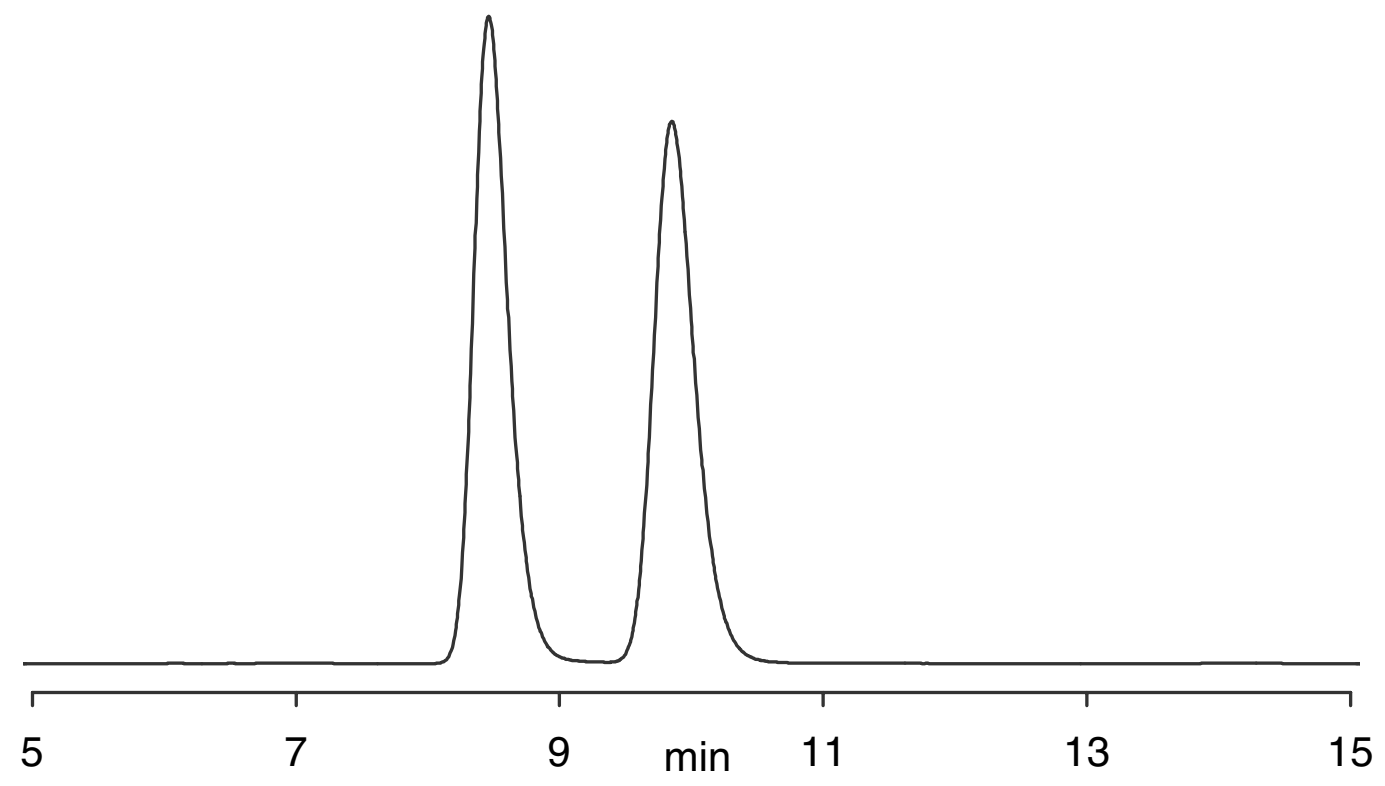

Figure S3. Optical resolution of alcohol 25 by CSP HPLC (Chiralcel OD column, $4.6 \mathrm{~mm} \times 25$ $\mathrm{cm}$; 2-propanol/hexanes, $15 / 85 ; 1 \mathrm{~mL} \mathrm{~min}^{-1} ; 40{ }^{\circ} \mathrm{C} ; 230 \mathrm{~nm}$ ). 

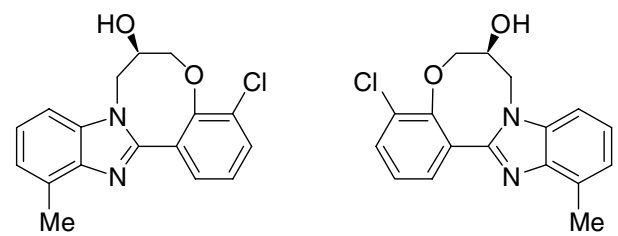

26

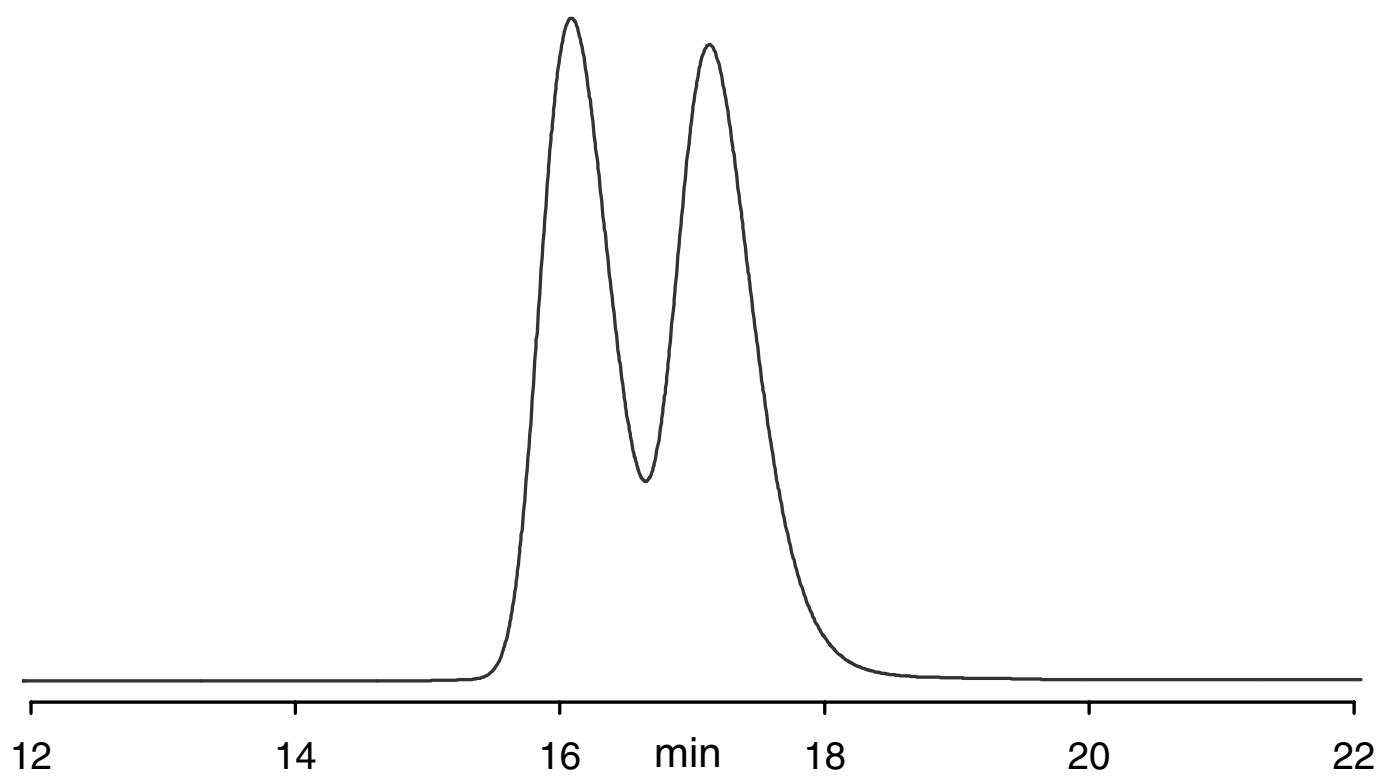

Figure S4. Optical resolution of alcohol 26 by CSP HPLC (Chiralcel OD column, $4.6 \mathrm{~mm} \times 25$ $\mathrm{cm}$; 2-propanol/hexanes, $8 / 92 ; 1 \mathrm{~mL} \mathrm{~min}^{-1} ; 40{ }^{\circ} \mathrm{C} ; 230 \mathrm{~nm}$ ). 

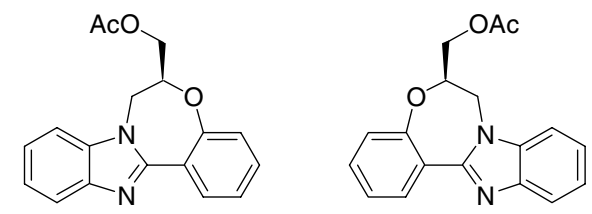

58

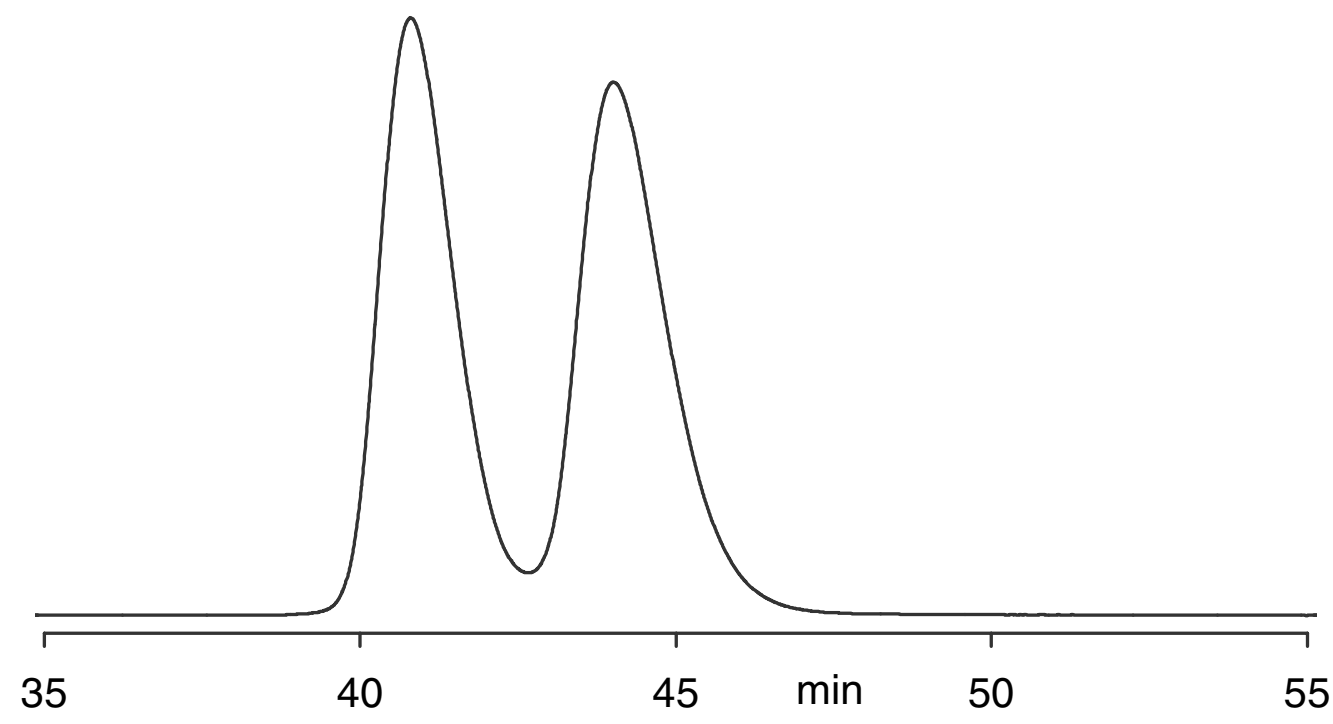

Figure S5. Optical resolution of acetate $\mathbf{5 8}$ by CSP HPLC (Chiralcel OD column, $4.6 \mathrm{~mm} \times 25$ $\mathrm{cm}$; 2-propanol/hexanes, 5/95; $\left.1 \mathrm{~mL} \mathrm{~min}^{-1} ; 40{ }^{\circ} \mathrm{C} ; 254 \mathrm{~nm}\right)$. 

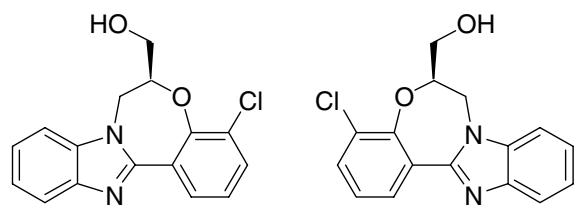

61

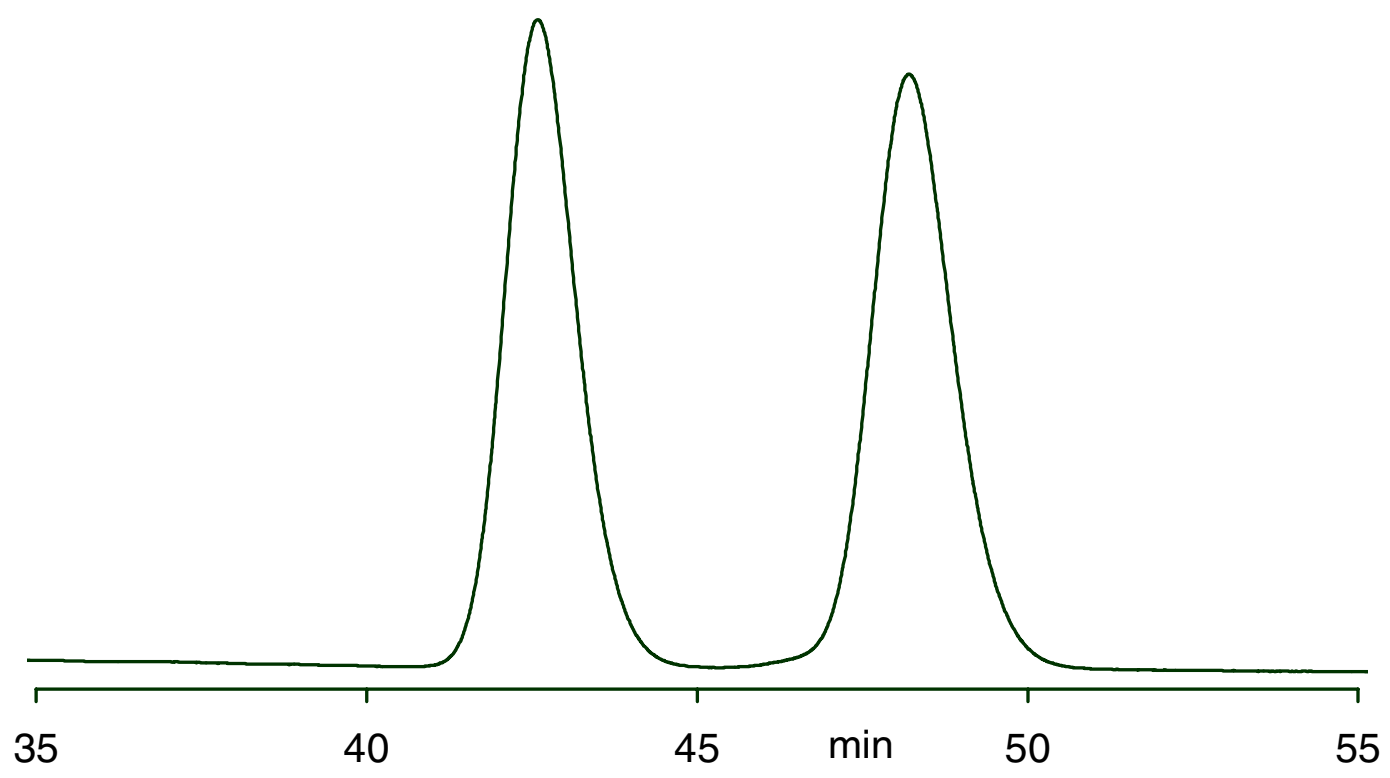

Figure S6. Optical resolution of alcohol $\mathbf{6 1}$ by CSP HPLC (Chiralcel OD column, $4.6 \mathrm{~mm} \times 25$ cm; 2-propanol/hexanes, $5 / 95 ; 1 \mathrm{~mL} \mathrm{~min}^{-1} ; 40{ }^{\circ} \mathrm{C} ; 254 \mathrm{~nm}$ ). 


\section{X-Ray Single-Crystal Data for Benzimidazole (9)}

The data collection crystal was a colorless, rectangular chunk. Examination of the diffraction pattern on a Nonius Kappa CCD diffractometer indicated a monoclinic crystal system. All work was done at $200 \mathrm{~K}$ using an Oxford Cryosystems Cryostream Cooler. The data collection strategy was set up to measure a quadrant of reciprocal space with a redundancy factor of 4 , meaning that $90 \%$ of the reflections were measured at least 4 times. A combination of phi and omega scans with a frame width of $1.0^{\circ}$ was used. Data integration was done with Denzo, ${ }^{13}$ and scaling and merging of the data was done with Scalepack. ${ }^{13}$ Merging the data and averaging the symmetry equivalent reflections resulted in an R(int) value of 0.043 . The teXsan package indicated the space group to be $P 2_{1} / n$, based on the systematic absences. ${ }^{14}$

The structure was solved by the direct methods in SHELXS-97. ${ }^{15}$ There are two molecules in the asymmetric unit and these are labeled as A and B. Full-matrix least-squares refinements based on $\mathrm{F}^{2}$ were performed in SHELXL-93. ${ }^{16}$

For each methyl group, the hydrogen atoms were added at calculated positions using a riding model with $\mathrm{U}(\mathrm{H})=1.5 \times \mathrm{Ueq}$ (bonded $\mathrm{C}$ atom). The torsion angle, which defines the orientation of the methyl group about the $\mathrm{C}-\mathrm{C}$ bond, was refined. The other hydrogen atoms were included in the model at calculated positions using a riding model with $\mathrm{U}(\mathrm{H})=1.2 \times$ Ueq (attached atom). The final refinement cycle was based on all 7009 intensities and 403 variables and resulted in agreement factors of $R_{1}(\mathrm{~F})=0.071$ and $\mathrm{w} R_{2}\left(\mathrm{~F}^{2}\right)=0.099$. For the subset of data with $I>2 \sigma(I)$, the $R_{1}(\mathrm{~F})$ value is 0.040 for 4857 reflections. The final difference electron density map contains maximum and minimum peak heights of 0.20 and $-0.17 \mathrm{e} / \AA^{3}$. Neutral atom scattering factors were used and include terms for anomalous dispersion. ${ }^{17}$

\footnotetext{
${ }^{13}$ DENZO: Otwinowski, Z.; Minor, W. in Methods in Enzymology; Carter Jr. C. W.; Sweet, R. M., Eds.; Academic Press, 1997, vol 276, pp 307-326.

14 teXsan: Crystal Structure Analysis Package, version 1.7-2, 1995; Molecular Structure Corporation, The Woodlands, TX.

15 SHELXS-97: Sheldrick, G. M. Universität Göttingen, Germany, 1997.

${ }^{16}$ SHELXL-93: Sheldrick, G. M. Universität Göttingen, Germany, 1993.

${ }^{17}$ International Tables for Crystallography, Volume C. Kluwer Academic Publishers: Dordrecht, 1992.
} 


\section{Table S1. Crystal Data Collection and Refinement Parameters for Benzimidazole (9).}

Empirical formula

Formula weight

Temperature

Wavelength

Crystal system

Space group

Unit cell dimensions

Volume

Z

Density (calculated)

Absorption coefficient

$\mathrm{F}(000)$

Crystal size

Theta range for data collection

Index ranges

Reflections collected

Independent reflections

Refinement method

Data / restraints / parameters

Goodness-of-fit on $\mathrm{F}^{2}$

Final R indices $[I>2 \sigma(I)]$

$\mathrm{R}$ indices (all data)

Largest diff. peak and hole
$\mathrm{C}_{19} \mathrm{H}_{20} \mathrm{~N}_{2} \mathrm{O}$

292.37

200(2) K

$0.71073 \AA$

monoclinic

$P 2{ }_{1} / n$

$a=13.088(1) \AA$

$b=10.022(1) \AA$

$c=24.198(3) \AA$

$\beta=105.15(1)^{\circ}$

3063.7(6) $\AA^{3}$

8

$1.268 \mathrm{Mg} / \mathrm{m}^{3}$

$0.079 \mathrm{~mm}^{-1}$

1248

$0.15 \times 0.23 \times 0.38 \mathrm{~mm}$

2.21 to $27.50^{\circ}$

$-16 \leq \mathrm{h} \leq 17,-13 \leq \mathrm{k} \leq 12,-31 \leq 1 \leq 31$

57018

$7009[\mathrm{R}($ int $)=0.043]$

Full-matrix least-squares on $\mathrm{F}^{2}$

7009 / 0 / 403

1.034

$R_{1}=0.0400, \mathrm{w} R_{2}=0.0883$

$R_{1}=0.0708, \mathrm{w} R_{2}=0.0991$

0.203 and $-0.172 \mathrm{e} / \AA^{3}$ 


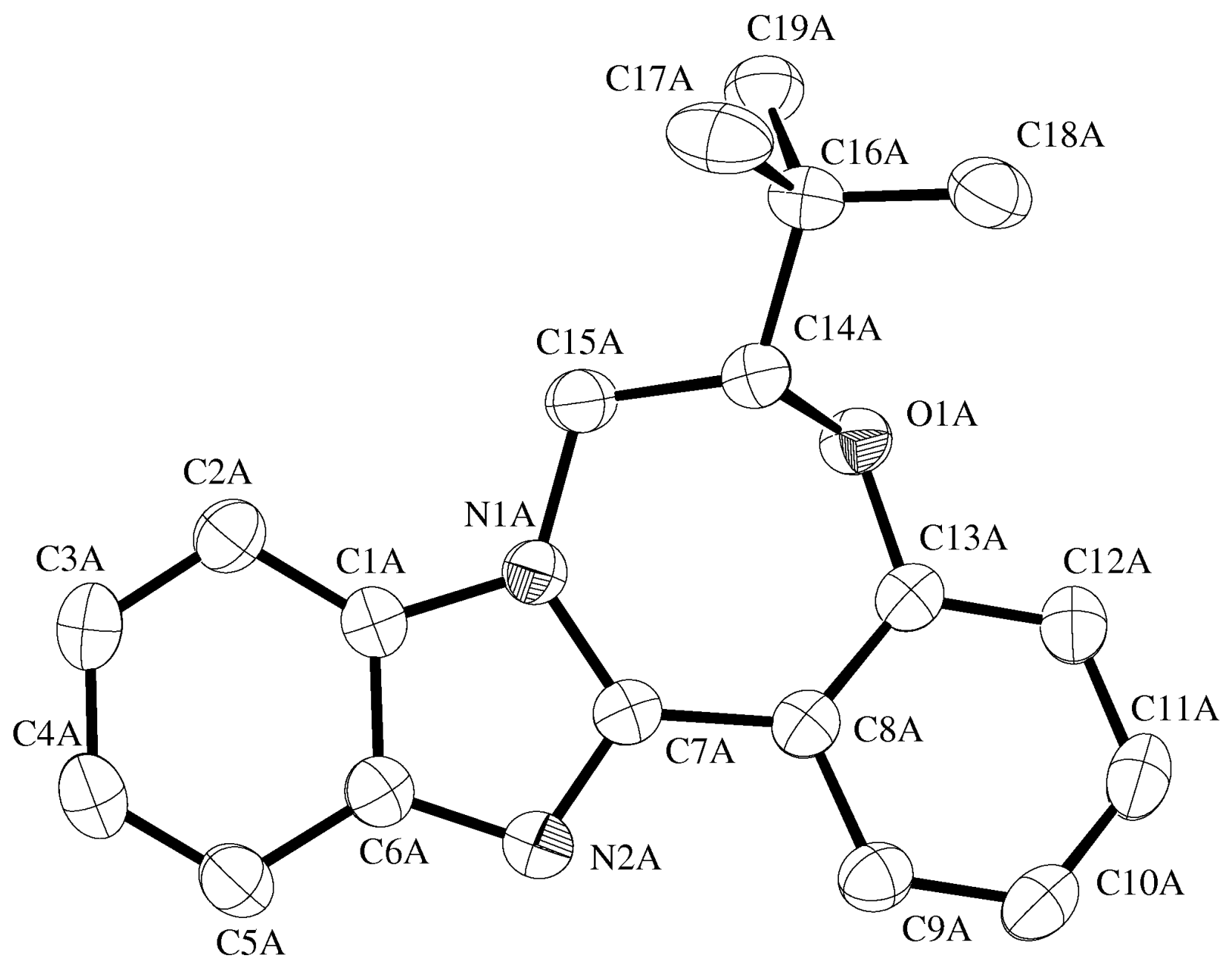

Figure S7. ORTEP drawing (50\% probability thermal ellipsoids) of the molecular structure of benzimidazole 9 . 

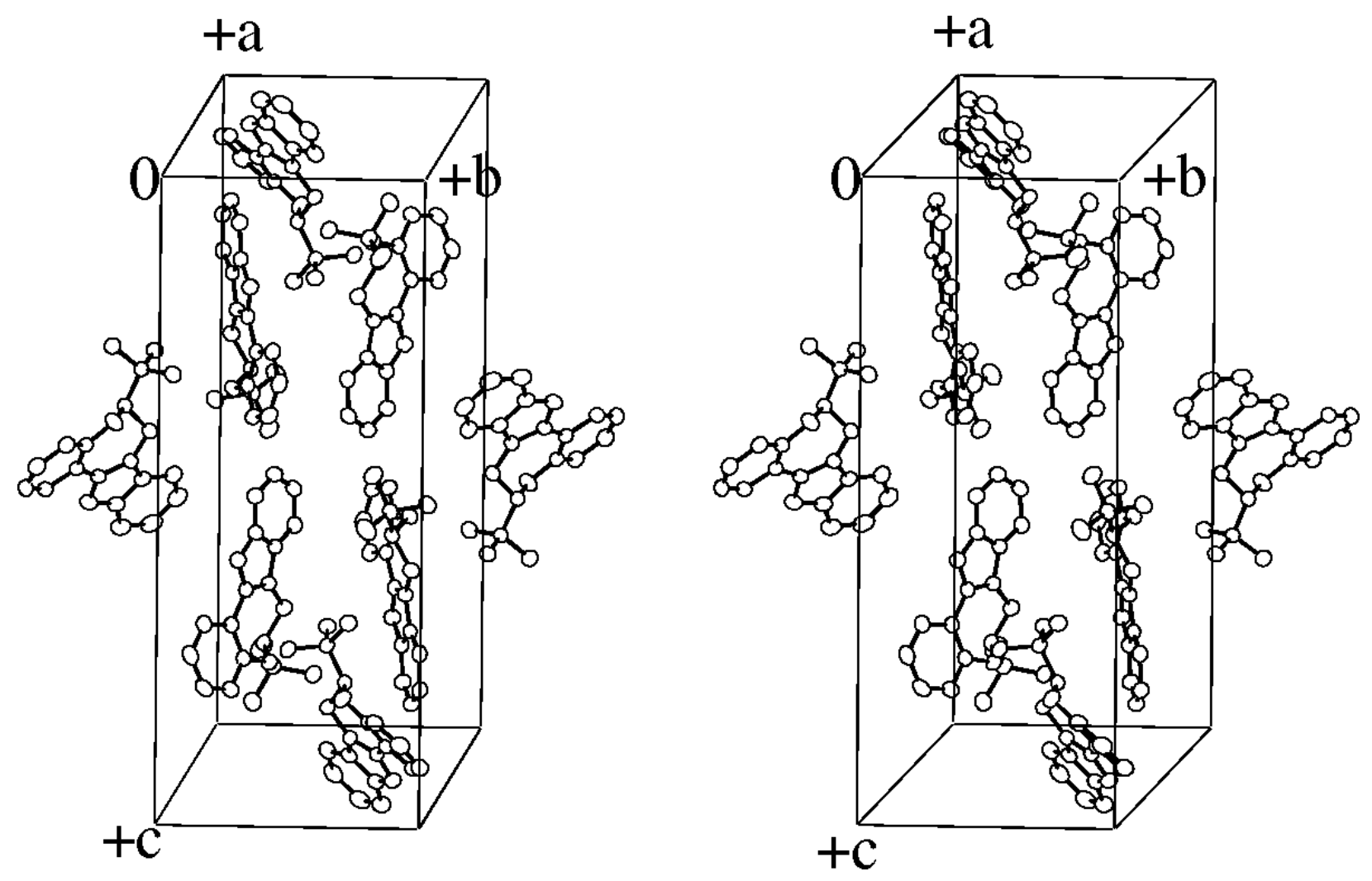

Figure S8. Packing diagram of benzimidazole 9. 
Table S2. Atomic Coordinates $\left[(x, y, z), \times 10^{4}\right]$ and Equivalent Isotropic Displacement U(eq) Parameters $\left(\AA^{2} \times 10^{3}\right)$ for Benzimidazole (9).

\begin{tabular}{|c|c|c|c|c|}
\hline & $\mathrm{x}$ & $\mathrm{y}$ & z & $\mathrm{U}(\mathrm{eq})^{18}$ \\
\hline $\mathrm{O}(1 \mathrm{~A})$ & $3033(1)$ & $7327(1)$ & $1437(1)$ & $34(1)$ \\
\hline $\mathrm{N}(1 \mathrm{~A})$ & $2878(1)$ & $7427(1)$ & $2629(1)$ & $31(1)$ \\
\hline $\mathrm{N}(2 \mathrm{~A})$ & $4367(1)$ & $8258(1)$ & $3215(1)$ & $36(1)$ \\
\hline$C(1 \mathrm{~A})$ & 2804(1) & $7217(1)$ & $3185(1)$ & $31(1)$ \\
\hline $\mathrm{C}(2 \mathrm{~A})$ & 2010(1) & $6667(1)$ & $3401(1)$ & $37(1)$ \\
\hline$C(3 A)$ & $2178(1)$ & $6683(1)$ & $3989(1)$ & $41(1)$ \\
\hline $\mathrm{C}(4 \mathrm{~A})$ & $3104(1)$ & $7206(1)$ & $4349(1)$ & $42(1)$ \\
\hline $\mathrm{C}(5 \mathrm{~A})$ & $3893(1)$ & $7727(1)$ & $4134(1)$ & $41(1)$ \\
\hline$C(6 A)$ & $3734(1)$ & 7744(1) & $3540(1)$ & $34(1)$ \\
\hline $\mathrm{C}(7 \mathrm{~A})$ & $3838(1)$ & 8061(1) & $2677(1)$ & $31(1)$ \\
\hline $\mathrm{C}(8 \mathrm{~A})$ & $4285(1)$ & $8497(1)$ & $2206(1)$ & $31(1)$ \\
\hline $\mathrm{C}(9 \mathrm{~A})$ & $5183(1)$ & $9320(1)$ & 2351(1) & $37(1)$ \\
\hline$C(10 A)$ & 5701(1) & $9707(1)$ & $1948(1)$ & $42(1)$ \\
\hline $\mathrm{C}(11 \mathrm{~A})$ & 5351(1) & $9255(1)$ & $1390(1)$ & $42(1)$ \\
\hline $\mathrm{C}(12 \mathrm{~A})$ & $4463(1)$ & $8461(1)$ & $1235(1)$ & $37(1)$ \\
\hline$C(13 \mathrm{~A})$ & 3921(1) & $8107(1)$ & $1633(1)$ & $31(1)$ \\
\hline $\mathrm{C}(14 \mathrm{~A})$ & $2102(1)$ & $7783(1)$ & $1596(1)$ & $31(1)$ \\
\hline$C(15 A)$ & $2046(1)$ & $7022(1)$ & $2125(1)$ & $35(1)$ \\
\hline$C(16 A)$ & $1136(1)$ & $7624(1)$ & $1076(1)$ & $35(1)$ \\
\hline $\mathrm{C}(17 \mathrm{~A})$ & $189(1)$ & $8306(2)$ & $1217(1)$ & $54(1)$ \\
\hline $\mathrm{C}(18 \mathrm{~A})$ & $1373(1)$ & $8337(2)$ & $564(1)$ & $49(1)$ \\
\hline $\mathrm{C}(19 \mathrm{~A})$ & $888(1)$ & 6161(1) & $929(1)$ & $41(1)$ \\
\hline $\mathrm{O}(1 \mathrm{~B})$ & $6688(1)$ & $3706(1)$ & $1469(1)$ & $41(1)$ \\
\hline $\mathrm{N}(1 \mathrm{~B})$ & 4651(1) & $3868(1)$ & $547(1)$ & $31(1)$ \\
\hline $\mathrm{N}(2 \mathrm{~B})$ & $4516(1)$ & $2270(1)$ & $-118(1)$ & $38(1)$ \\
\hline $\mathrm{C}(1 \mathrm{~B})$ & $3726(1)$ & $4120(1)$ & 130(1) & $33(1)$ \\
\hline
\end{tabular}

\footnotetext{
${ }^{18} \mathrm{U}(\mathrm{eq})$ is defined as one third of the trace of the orthogonalized Uij tensor.
} 


$\begin{array}{lllrr}\mathrm{C}(2 \mathrm{~B}) & 2971(1) & 5117(1) & 82(1) & 40(1) \\ \mathrm{C}(3 \mathrm{~B}) & 2128(1) & 5076(2) & -399(1) & 47(1) \\ \mathrm{C}(4 \mathrm{~B}) & 2044(1) & 4082(2) & -814(1) & 49(1) \\ \mathrm{C}(5 \mathrm{~B}) & 2793(1) & 3094(2) & -763(1) & 45(1) \\ \mathrm{C}(6 \mathrm{~B}) & 3653(1) & 3113(1) & -277(1) & 36(1) \\ \mathrm{C}(7 \mathrm{~B}) & 5101(1) & 2748(1) & 374(1) & 31(1) \\ \mathrm{C}(8 B) & 6141(1) & 2173(1) & 654(1) & 32(1) \\ \mathrm{C}(9 B) & 6465(1) & 1074(1) & 378(1) & 39(1) \\ \mathrm{C}(10 B) & 7473(1) & 570(1) & 550(1) & 47(1) \\ \mathrm{C}(11 B) & 8212(1) & 1156(1) & 1002(1) & 47(1) \\ \mathrm{C}(12 B) & 7909(1) & 2196(1) & 1295(1) & 40(1) \\ \mathrm{C}(13 B) & 6875(1) & 2677(1) & 1134(1) & 33(1) \\ \mathrm{C}(14 B) & 5653(1) & 3863(1) & 1557(1) & 31(1) \\ \mathrm{C}(15 B) & 4980(1) & 4659(1) & 1069(1) & 38(1) \\ \mathrm{C}(16 B) & 5817(1) & 4520(1) & 2150(1) & 33(1) \\ \mathrm{C}(17 B) & 4742(1) & 4759(1) & 2276(1) & 42(1) \\ \mathrm{C}(18 B) & 6464(1) & 3562(1) & 2602(1) & 44(1) \\ \mathrm{C}(19 B) & 6406(1) & 5847(1) & 2172(1) & 41(1)\end{array}$


Table S3. Bond Lengths $[\AA ̊]$ and Angles $\left[{ }^{\circ}\right]$ for Benzimidazole (9).

\begin{tabular}{|c|c|}
\hline $\mathrm{O}(1 \mathrm{~A})-\mathrm{C}(13 \mathrm{~A})$ & $1.378(1)$ \\
\hline $\mathrm{O}(1 \mathrm{~A})-\mathrm{C}(14 \mathrm{~A})$ & $1.444(1)$ \\
\hline $\mathrm{N}(1 \mathrm{~A})-\mathrm{C}(7 \mathrm{~A})$ & $1.386(2)$ \\
\hline $\mathrm{N}(1 \mathrm{~A})-\mathrm{C}(1 \mathrm{~A})$ & $1.390(2)$ \\
\hline $\mathrm{N}(1 \mathrm{~A})-\mathrm{C}(15 \mathrm{~A})$ & $1.464(2)$ \\
\hline $\mathrm{N}(2 \mathrm{~A})-\mathrm{C}(7 \mathrm{~A})$ & $1.321(2)$ \\
\hline $\mathrm{N}(2 \mathrm{~A})-\mathrm{C}(6 \mathrm{~A})$ & $1.382(2)$ \\
\hline $\mathrm{C}(1 \mathrm{~A})-\mathrm{C}(2 \mathrm{~A})$ & $1.393(2)$ \\
\hline$C(1 A)-C(6 A)$ & $1.397(2)$ \\
\hline$C(2 A)-C(3 A)$ & $1.383(2)$ \\
\hline $\mathrm{C}(2 \mathrm{~A})-\mathrm{H}(2 \mathrm{~A})$ & 0.95 \\
\hline$C(3 A)-C(4 A)$ & $1.397(2)$ \\
\hline $\mathrm{C}(3 \mathrm{~A})-\mathrm{H}(3 \mathrm{~A})$ & 0.95 \\
\hline$C(4 A)-C(5 A)$ & $1.376(2)$ \\
\hline $\mathrm{C}(4 \mathrm{~A})-\mathrm{H}(4 \mathrm{~A})$ & 0.95 \\
\hline$C(5 A)-C(6 A)$ & $1.396(2)$ \\
\hline $\mathrm{C}(5 \mathrm{~A})-\mathrm{H}(5 \mathrm{~A})$ & 0.95 \\
\hline $\mathrm{C}(7 \mathrm{~A})-\mathrm{C}(8 \mathrm{~A})$ & $1.477(2)$ \\
\hline $\mathrm{C}(8 \mathrm{~A})-\mathrm{C}(13 \mathrm{~A})$ & $1.398(2)$ \\
\hline$C(8 A)-C(9 A)$ & $1.403(2)$ \\
\hline $\mathrm{C}(9 \mathrm{~A})-\mathrm{C}(10 \mathrm{~A})$ & $1.381(2)$ \\
\hline $\mathrm{C}(9 \mathrm{~A})-\mathrm{H}(9 \mathrm{~A})$ & 0.95 \\
\hline$C(10 A)-C(11 A)$ & $1.384(2)$ \\
\hline $\mathrm{C}(10 \mathrm{~A})-\mathrm{H}(10 \mathrm{~A})$ & 0.95 \\
\hline $\mathrm{C}(11 \mathrm{~A})-\mathrm{C}(12 \mathrm{~A})$ & $1.377(2)$ \\
\hline $\mathrm{C}(11 \mathrm{~A})-\mathrm{H}(11 \mathrm{~A})$ & 0.95 \\
\hline $\mathrm{C}(12 \mathrm{~A})-\mathrm{C}(13 \mathrm{~A})$ & $1.384(2)$ \\
\hline $\mathrm{C}(12 \mathrm{~A})-\mathrm{H}(12 \mathrm{~A})$ & 0.95 \\
\hline$C(14 A)-C(15 A)$ & $1.508(2)$ \\
\hline
\end{tabular}




\begin{tabular}{|c|c|}
\hline$C(14 A)-C(16 A)$ & $1.542(2)$ \\
\hline $\mathrm{C}(14 \mathrm{~A})-\mathrm{H}(14 \mathrm{~A})$ & 1.00 \\
\hline $\mathrm{C}(15 \mathrm{~A})-\mathrm{H}(15 \mathrm{~A})$ & 0.99 \\
\hline $\mathrm{C}(15 \mathrm{~A})-\mathrm{H}(15 \mathrm{~B})$ & 0.99 \\
\hline$C(16 A)-C(19 A)$ & $1.525(2)$ \\
\hline$C(16 A)-C(17 A)$ & $1.529(2)$ \\
\hline $\mathrm{C}(16 \mathrm{~A})-\mathrm{C}(18 \mathrm{~A})$ & $1.531(2)$ \\
\hline $\mathrm{C}(17 \mathrm{~A})-\mathrm{H}(17 \mathrm{~A})$ & 0.98 \\
\hline $\mathrm{C}(17 \mathrm{~A})-\mathrm{H}(17 \mathrm{~B})$ & 0.98 \\
\hline $\mathrm{C}(17 \mathrm{~A})-\mathrm{H}(17 \mathrm{C})$ & 0.98 \\
\hline $\mathrm{C}(18 \mathrm{~A})-\mathrm{H}(18 \mathrm{~A})$ & 0.98 \\
\hline $\mathrm{C}(18 \mathrm{~A})-\mathrm{H}(18 \mathrm{~B})$ & 0.98 \\
\hline $\mathrm{C}(18 \mathrm{~A})-\mathrm{H}(18 \mathrm{C})$ & 0.98 \\
\hline $\mathrm{C}(19 \mathrm{~A})-\mathrm{H}(19 \mathrm{~A})$ & 0.98 \\
\hline C(19A)-H(19B) & 0.98 \\
\hline $\mathrm{C}(19 \mathrm{~A})-\mathrm{H}(19 \mathrm{C})$ & 0.98 \\
\hline $\mathrm{O}(1 \mathrm{~B})-\mathrm{C}(13 \mathrm{~B})$ & $1.373(1)$ \\
\hline $\mathrm{O}(1 \mathrm{~B})-\mathrm{C}(14 \mathrm{~B})$ & $1.433(1)$ \\
\hline $\mathrm{N}(1 \mathrm{~B})-\mathrm{C}(7 \mathrm{~B})$ & $1.381(2)$ \\
\hline $\mathrm{N}(1 \mathrm{~B})-\mathrm{C}(1 \mathrm{~B})$ & $1.381(2)$ \\
\hline $\mathrm{N}(1 \mathrm{~B})-\mathrm{C}(15 \mathrm{~B})$ & $1.459(2)$ \\
\hline $\mathrm{N}(2 \mathrm{~B})-\mathrm{C}(7 \mathrm{~B})$ & $1.324(2)$ \\
\hline$N(2 B)-C(6 B)$ & $1.382(2)$ \\
\hline $\mathrm{C}(1 \mathrm{~B})-\mathrm{C}(2 \mathrm{~B})$ & $1.388(2)$ \\
\hline $\mathrm{C}(1 \mathrm{~B})-\mathrm{C}(6 \mathrm{~B})$ & $1.396(2)$ \\
\hline $\mathrm{C}(2 \mathrm{~B})-\mathrm{C}(3 \mathrm{~B})$ & $1.379(2)$ \\
\hline $\mathrm{C}(2 \mathrm{~B})-\mathrm{H}(2 \mathrm{~B})$ & 0.95 \\
\hline $\mathrm{C}(3 \mathrm{~B})-\mathrm{C}(4 \mathrm{~B})$ & $1.398(2)$ \\
\hline $\mathrm{C}(3 \mathrm{~B})-\mathrm{H}(3 \mathrm{~B})$ & 0.95 \\
\hline $\mathrm{C}(4 \mathrm{~B})-\mathrm{C}(5 \mathrm{~B})$ & $1.375(2)$ \\
\hline $\mathrm{C}(4 \mathrm{~B})-\mathrm{H}(4 \mathrm{~B})$ & 0.95 \\
\hline
\end{tabular}




\begin{tabular}{|c|c|}
\hline$C(5 B)-C(6 B)$ & $1.399(2)$ \\
\hline $\mathrm{C}(5 \mathrm{~B})-\mathrm{H}(5 \mathrm{~B})$ & 0.95 \\
\hline$C(7 B)-C(8 B)$ & $1.471(2)$ \\
\hline $\mathrm{C}(8 \mathrm{~B})-\mathrm{C}(13 \mathrm{~B})$ & $1.394(2)$ \\
\hline $\mathrm{C}(8 \mathrm{~B})-\mathrm{C}(9 \mathrm{~B})$ & $1.410(2)$ \\
\hline $\mathrm{C}(9 \mathrm{~B})-\mathrm{C}(10 \mathrm{~B})$ & $1.371(2)$ \\
\hline $\mathrm{C}(9 \mathrm{~B})-\mathrm{H}(9 \mathrm{~B})$ & 0.95 \\
\hline$C(10 B)-C(11 B)$ & $1.387(2)$ \\
\hline $\mathrm{C}(10 \mathrm{~B})-\mathrm{H}(10 \mathrm{~B})$ & 0.95 \\
\hline $\mathrm{C}(11 \mathrm{~B})-\mathrm{C}(12 \mathrm{~B})$ & $1.375(2)$ \\
\hline $\mathrm{C}(11 \mathrm{~B})-\mathrm{H}(11 \mathrm{~B})$ & 0.95 \\
\hline$C(12 B)-C(13 B)$ & $1.393(2)$ \\
\hline $\mathrm{C}(12 \mathrm{~B})-\mathrm{H}(12 \mathrm{~B})$ & 0.95 \\
\hline $\mathrm{C}(14 \mathrm{~B})-\mathrm{C}(15 \mathrm{~B})$ & $1.505(2)$ \\
\hline$C(14 B)-C(16 B)$ & $1.542(2)$ \\
\hline $\mathrm{C}(14 \mathrm{~B})-\mathrm{H}(14 \mathrm{~B})$ & 1.00 \\
\hline $\mathrm{C}(15 \mathrm{~B})-\mathrm{H}(15 \mathrm{C})$ & 0.99 \\
\hline $\mathrm{C}(15 \mathrm{~B})-\mathrm{H}(15 \mathrm{D})$ & 0.99 \\
\hline$C(16 B)-C(19 B)$ & $1.532(2)$ \\
\hline$C(16 B)-C(17 B)$ & $1.534(2)$ \\
\hline$C(16 B)-C(18 B)$ & $1.534(2)$ \\
\hline $\mathrm{C}(17 \mathrm{~B})-\mathrm{H}(17 \mathrm{D})$ & 0.98 \\
\hline $\mathrm{C}(17 \mathrm{~B})-\mathrm{H}(17 \mathrm{E})$ & 0.98 \\
\hline $\mathrm{C}(17 \mathrm{~B})-\mathrm{H}(17 \mathrm{~F})$ & 0.98 \\
\hline $\mathrm{C}(18 \mathrm{~B})-\mathrm{H}(18 \mathrm{D})$ & 0.98 \\
\hline $\mathrm{C}(18 \mathrm{~B})-\mathrm{H}(18 \mathrm{E})$ & 0.98 \\
\hline $\mathrm{C}(18 \mathrm{~B})-\mathrm{H}(18 \mathrm{~F})$ & 0.98 \\
\hline C(19B)-H(19D) & 0.98 \\
\hline C(19B)-H(19E) & 0.98 \\
\hline $\mathrm{C}(19 \mathrm{~B})-\mathrm{H}(19 \mathrm{~F})$ & 0.98 \\
\hline
\end{tabular}




\begin{tabular}{|c|c|}
\hline $\mathrm{C}(13 \mathrm{~A})-\mathrm{O}(1 \mathrm{~A})-\mathrm{C}(14 \mathrm{~A})$ & $115.06(9)$ \\
\hline$C(7 A)-N(1 A)-C(1 A)$ & $106.30(9)$ \\
\hline $\mathrm{C}(7 \mathrm{~A})-\mathrm{N}(1 \mathrm{~A})-\mathrm{C}(15 \mathrm{~A})$ & $131.07(10)$ \\
\hline $\mathrm{C}(1 \mathrm{~A})-\mathrm{N}(1 \mathrm{~A})-\mathrm{C}(15 \mathrm{~A})$ & $122.63(10)$ \\
\hline$C(7 A)-N(2 A)-C(6 A)$ & $105.60(10)$ \\
\hline $\mathrm{N}(1 \mathrm{~A})-\mathrm{C}(1 \mathrm{~A})-\mathrm{C}(2 \mathrm{~A})$ & $132.02(11)$ \\
\hline$N(1 A)-C(1 A)-C(6 A)$ & $105.55(10)$ \\
\hline$C(2 A)-C(1 A)-C(6 A)$ & $122.39(11)$ \\
\hline$C(3 A)-C(2 A)-C(1 A)$ & $116.55(12)$ \\
\hline $\mathrm{C}(3 \mathrm{~A})-\mathrm{C}(2 \mathrm{~A})-\mathrm{H}(2 \mathrm{~A})$ & 121.7 \\
\hline $\mathrm{C}(1 \mathrm{~A})-\mathrm{C}(2 \mathrm{~A})-\mathrm{H}(2 \mathrm{~A})$ & 121.7 \\
\hline $\mathrm{C}(2 \mathrm{~A})-\mathrm{C}(3 \mathrm{~A})-\mathrm{C}(4 \mathrm{~A})$ & $121.67(12)$ \\
\hline $\mathrm{C}(2 \mathrm{~A})-\mathrm{C}(3 \mathrm{~A})-\mathrm{H}(3 \mathrm{~A})$ & 119.2 \\
\hline $\mathrm{C}(4 \mathrm{~A})-\mathrm{C}(3 \mathrm{~A})-\mathrm{H}(3 \mathrm{~A})$ & 119.2 \\
\hline$C(5 A)-C(4 A)-C(3 A)$ & $121.45(12)$ \\
\hline $\mathrm{C}(5 \mathrm{~A})-\mathrm{C}(4 \mathrm{~A})-\mathrm{H}(4 \mathrm{~A})$ & 119.3 \\
\hline $\mathrm{C}(3 \mathrm{~A})-\mathrm{C}(4 \mathrm{~A})-\mathrm{H}(4 \mathrm{~A})$ & 119.3 \\
\hline$C(4 A)-C(5 A)-C(6 A)$ & $117.97(13)$ \\
\hline $\mathrm{C}(4 \mathrm{~A})-\mathrm{C}(5 \mathrm{~A})-\mathrm{H}(5 \mathrm{~A})$ & 121.0 \\
\hline $\mathrm{C}(6 \mathrm{~A})-\mathrm{C}(5 \mathrm{~A})-\mathrm{H}(5 \mathrm{~A})$ & 121.0 \\
\hline$N(2 A)-C(6 A)-C(5 A)$ & $129.75(12)$ \\
\hline$N(2 A)-C(6 A)-C(1 A)$ & $110.26(11)$ \\
\hline$C(5 A)-C(6 A)-C(1 A)$ & $119.97(12)$ \\
\hline $\mathrm{N}(2 \mathrm{~A})-\mathrm{C}(7 \mathrm{~A})-\mathrm{N}(1 \mathrm{~A})$ & $112.30(10)$ \\
\hline $\mathrm{N}(2 \mathrm{~A})-\mathrm{C}(7 \mathrm{~A})-\mathrm{C}(8 \mathrm{~A})$ & $120.41(11)$ \\
\hline $\mathrm{N}(1 \mathrm{~A})-\mathrm{C}(7 \mathrm{~A})-\mathrm{C}(8 \mathrm{~A})$ & $127.29(11)$ \\
\hline $\mathrm{C}(13 \mathrm{~A})-\mathrm{C}(8 \mathrm{~A})-\mathrm{C}(9 \mathrm{~A})$ & $117.33(11)$ \\
\hline $\mathrm{C}(13 \mathrm{~A})-\mathrm{C}(8 \mathrm{~A})-\mathrm{C}(7 \mathrm{~A})$ & $125.49(11)$ \\
\hline$C(9 A)-C(8 A)-C(7 A)$ & $117.10(11)$ \\
\hline$C(10 A)-C(9 A)-C(8 A)$ & $121.44(12)$ \\
\hline $\mathrm{C}(10 \mathrm{~A})-\mathrm{C}(9 \mathrm{~A})-\mathrm{H}(9 \mathrm{~A})$ & 119.3 \\
\hline
\end{tabular}




$\begin{array}{ll}\mathrm{C}(8 \mathrm{~A})-\mathrm{C}(9 \mathrm{~A})-\mathrm{H}(9 \mathrm{~A}) & 119.3 \\ \mathrm{C}(9 \mathrm{~A})-\mathrm{C}(10 \mathrm{~A})-\mathrm{C}(11 \mathrm{~A}) & 119.92(13) \\ \mathrm{C}(9 \mathrm{~A})-\mathrm{C}(10 \mathrm{~A})-\mathrm{H}(10 \mathrm{~A}) & 120.0 \\ \mathrm{C}(11 \mathrm{~A})-\mathrm{C}(10 \mathrm{~A})-\mathrm{H}(10 \mathrm{~A}) & 120.0 \\ \mathrm{C}(12 \mathrm{~A})-\mathrm{C}(11 \mathrm{~A})-\mathrm{C}(10 \mathrm{~A}) & 119.71(12) \\ \mathrm{C}(12 \mathrm{~A})-\mathrm{C}(11 \mathrm{~A})-\mathrm{H}(11 \mathrm{~A}) & 120.1 \\ \mathrm{C}(10 \mathrm{~A})-\mathrm{C}(11 \mathrm{~A})-\mathrm{H}(11 \mathrm{~A}) & 120.1 \\ \mathrm{C}(11 \mathrm{~A})-\mathrm{C}(12 \mathrm{~A})-\mathrm{C}(13 \mathrm{~A}) & 120.53(12) \\ \mathrm{C}(11 \mathrm{~A})-\mathrm{C}(12 \mathrm{~A})-\mathrm{H}(12 \mathrm{~A}) & 119.7 \\ \mathrm{C}(13 \mathrm{~A})-\mathrm{C}(12 \mathrm{~A})-\mathrm{H}(12 \mathrm{~A}) & 119.7 \\ \mathrm{O}(1 \mathrm{~A})-\mathrm{C}(13 \mathrm{~A})-\mathrm{C}(12 \mathrm{~A}) & 116.47(11) \\ \mathrm{O}(1 \mathrm{~A})-\mathrm{C}(13 \mathrm{~A})-\mathrm{C}(8 \mathrm{~A}) & 122.54(10) \\ \mathrm{C}(12 \mathrm{~A})-\mathrm{C}(13 \mathrm{~A})-\mathrm{C}(8 \mathrm{~A}) & 120.96(11) \\ \mathrm{O}(1 \mathrm{~A})-\mathrm{C}(14 \mathrm{~A})-\mathrm{C}(15 \mathrm{~A}) & 107.36(10) \\ \mathrm{O}(1 \mathrm{~A})-\mathrm{C}(14 \mathrm{~A})-\mathrm{C}(16 \mathrm{~A}) & 108.60(9) \\ \mathrm{C}(15 \mathrm{~A})-\mathrm{C}(14 \mathrm{~A})-\mathrm{C}(16 \mathrm{~A}) & 115.38(10) \\ \mathrm{O}(1 \mathrm{~A})-\mathrm{C}(14 \mathrm{~A})-\mathrm{H}(14 \mathrm{~A}) & 108.4 \\ \mathrm{C}(15 \mathrm{~A})-\mathrm{C}(14 \mathrm{~A})-\mathrm{H}(14 \mathrm{~A}) & 108.4 \\ \mathrm{C}(16 \mathrm{~A})-\mathrm{C}(14 \mathrm{~A})-\mathrm{H}(14 \mathrm{~A}) & 108.4 \\ \mathrm{~N}(1 \mathrm{~A})-\mathrm{C}(15 \mathrm{~A})-\mathrm{C}(14 \mathrm{~A}) & 112.05(10) \\ \mathrm{N}(1 \mathrm{~A})-\mathrm{C}(15 \mathrm{~A})-\mathrm{H}(15 \mathrm{~A}) & 109.2 \\ \mathrm{C}(14 \mathrm{~A})-\mathrm{C}(15 \mathrm{~A})-\mathrm{H}(15 \mathrm{~A}) & 109.2 \\ \mathrm{~N}(1 \mathrm{~A})-\mathrm{C}(15 \mathrm{~A})-\mathrm{H}(15 \mathrm{~B}) & 109.2 \\ \mathrm{C}(14 \mathrm{~A})-\mathrm{C}(15 \mathrm{~A})-\mathrm{H}(15 \mathrm{~B}) & 109.2 \\ \mathrm{H}(15 \mathrm{~A})-\mathrm{C}(15 \mathrm{~A})-\mathrm{H}(15 \mathrm{~B}) & 107.9 \\ \mathrm{C}(19 \mathrm{~A})-\mathrm{C}(16 \mathrm{~A})-\mathrm{C}(17 \mathrm{~A}) & 110.34(11) \\ \mathrm{C}(19 \mathrm{~A})-\mathrm{C}(16 \mathrm{~A})-\mathrm{C}(18 \mathrm{~A}) & 109.55(11) \\ \mathrm{C}(17 \mathrm{~A})-\mathrm{C}(16 \mathrm{~A})-\mathrm{C}(18 \mathrm{~A}) & 108.61(12) \\ \mathrm{C}(19 \mathrm{~A})-\mathrm{C}(16 \mathrm{~A})-\mathrm{C}(14 \mathrm{~A}) & 111.66(10) \\ \mathrm{C}(17 \mathrm{~A})-\mathrm{C}(16 \mathrm{~A})-\mathrm{C}(14 \mathrm{~A})-\mathrm{A}(16 \mathrm{~A})-\mathrm{C}(14 \mathrm{~A}) & 108.17(10) \\ & \\ & 108.43(10) \\ & \end{array}$




\begin{tabular}{|c|c|}
\hline$C(16 A)-C(17 A)-H(17 A)$ & 109.5 \\
\hline $\mathrm{C}(16 \mathrm{~A})-\mathrm{C}(17 \mathrm{~A})-\mathrm{H}(17 \mathrm{~B})$ & 109.5 \\
\hline $\mathrm{H}(17 \mathrm{~A})-\mathrm{C}(17 \mathrm{~A})-\mathrm{H}(17 \mathrm{~B})$ & 109.5 \\
\hline $\mathrm{C}(16 \mathrm{~A})-\mathrm{C}(17 \mathrm{~A})-\mathrm{H}(17 \mathrm{C})$ & 109.5 \\
\hline $\mathrm{H}(17 \mathrm{~A})-\mathrm{C}(17 \mathrm{~A})-\mathrm{H}(17 \mathrm{C})$ & 109.5 \\
\hline $\mathrm{H}(17 \mathrm{~B})-\mathrm{C}(17 \mathrm{~A})-\mathrm{H}(17 \mathrm{C})$ & 109.5 \\
\hline $\mathrm{C}(16 \mathrm{~A})-\mathrm{C}(18 \mathrm{~A})-\mathrm{H}(18 \mathrm{~A})$ & 109.5 \\
\hline $\mathrm{C}(16 \mathrm{~A})-\mathrm{C}(18 \mathrm{~A})-\mathrm{H}(18 \mathrm{~B})$ & 109.5 \\
\hline $\mathrm{H}(18 \mathrm{~A})-\mathrm{C}(18 \mathrm{~A})-\mathrm{H}(18 \mathrm{~B})$ & 109.5 \\
\hline $\mathrm{C}(16 \mathrm{~A})-\mathrm{C}(18 \mathrm{~A})-\mathrm{H}(18 \mathrm{C})$ & 109.5 \\
\hline $\mathrm{H}(18 \mathrm{~A})-\mathrm{C}(18 \mathrm{~A})-\mathrm{H}(18 \mathrm{C})$ & 109.5 \\
\hline $\mathrm{H}(18 \mathrm{~B})-\mathrm{C}(18 \mathrm{~A})-\mathrm{H}(18 \mathrm{C})$ & 109.5 \\
\hline$C(16 A)-C(19 A)-H(19 A)$ & 109.5 \\
\hline $\mathrm{C}(16 \mathrm{~A})-\mathrm{C}(19 \mathrm{~A})-\mathrm{H}(19 \mathrm{~B})$ & 109.5 \\
\hline H(19A)-C(19A)-H(19B) & 109.5 \\
\hline$C(16 A)-C(19 A)-H(19 C)$ & 109.5 \\
\hline H(19A)-C(19A)-H(19C) & 109.5 \\
\hline H(19B)-C(19A)-H(19C) & 109.5 \\
\hline$C(13 B)-O(1 B)-C(14 B)$ & $119.43(9)$ \\
\hline $\mathrm{C}(7 \mathrm{~B})-\mathrm{N}(1 \mathrm{~B})-\mathrm{C}(1 \mathrm{~B})$ & $106.82(10)$ \\
\hline $\mathrm{C}(7 \mathrm{~B})-\mathrm{N}(1 \mathrm{~B})-\mathrm{C}(15 \mathrm{~B})$ & $130.67(10)$ \\
\hline$C(1 B)-N(1 B)-C(15 B)$ & $122.43(10)$ \\
\hline$C(7 B)-N(2 B)-C(6 B)$ & $105.52(10)$ \\
\hline $\mathrm{N}(1 \mathrm{~B})-\mathrm{C}(1 \mathrm{~B})-\mathrm{C}(2 \mathrm{~B})$ & $131.49(12)$ \\
\hline $\mathrm{N}(1 \mathrm{~B})-\mathrm{C}(1 \mathrm{~B})-\mathrm{C}(6 \mathrm{~B})$ & $105.52(11)$ \\
\hline$C(2 B)-C(1 B)-C(6 B)$ & $122.98(12)$ \\
\hline $\mathrm{C}(3 \mathrm{~B})-\mathrm{C}(2 \mathrm{~B})-\mathrm{C}(1 \mathrm{~B})$ & $116.41(13)$ \\
\hline $\mathrm{C}(3 \mathrm{~B})-\mathrm{C}(2 \mathrm{~B})-\mathrm{H}(2 \mathrm{~B})$ & 121.8 \\
\hline $\mathrm{C}(1 \mathrm{~B})-\mathrm{C}(2 \mathrm{~B})-\mathrm{H}(2 \mathrm{~B})$ & 121.8 \\
\hline$C(2 B)-C(3 B)-C(4 B)$ & $121.48(13)$ \\
\hline $\mathrm{C}(2 \mathrm{~B})-\mathrm{C}(3 \mathrm{~B})-\mathrm{H}(3 \mathrm{~B})$ & 119.3 \\
\hline
\end{tabular}




$\begin{array}{ll}\mathrm{C}(4 \mathrm{~B})-\mathrm{C}(3 \mathrm{~B})-\mathrm{H}(3 \mathrm{~B}) & 119.3 \\ \mathrm{C}(5 \mathrm{~B})-\mathrm{C}(4 \mathrm{~B})-\mathrm{C}(3 \mathrm{~B}) & 121.82(13) \\ \mathrm{C}(5 \mathrm{~B})-\mathrm{C}(4 \mathrm{~B})-\mathrm{H}(4 \mathrm{~B}) & 119.1 \\ \mathrm{C}(3 \mathrm{~B})-\mathrm{C}(4 \mathrm{~B})-\mathrm{H}(4 \mathrm{~B}) & 119.1 \\ \mathrm{C}(4 \mathrm{~B})-\mathrm{C}(5 \mathrm{~B})-\mathrm{C}(6 \mathrm{~B}) & 117.69(13) \\ \mathrm{C}(4 \mathrm{~B})-\mathrm{C}(5 \mathrm{~B})-\mathrm{H}(5 \mathrm{~B}) & 121.2 \\ \mathrm{C}(6 \mathrm{~B})-\mathrm{C}(5 \mathrm{~B})-\mathrm{H}(5 \mathrm{~B}) & 121.2 \\ \mathrm{~N}(2 \mathrm{~B})-\mathrm{C}(6 \mathrm{~B})-\mathrm{C}(1 \mathrm{~B}) & 110.16(11) \\ \mathrm{N}(2 \mathrm{~B})-\mathrm{C}(6 \mathrm{~B})-\mathrm{C}(5 \mathrm{~B}) & 130.22(12) \\ \mathrm{C}(1 \mathrm{~B})-\mathrm{C}(6 \mathrm{~B})-\mathrm{C}(5 \mathrm{~B}) & 119.61(13) \\ \mathrm{N}(2 \mathrm{~B})-\mathrm{C}(7 \mathrm{~B})-\mathrm{N}(1 \mathrm{~B}) & 111.97(11) \\ \mathrm{N}(2 \mathrm{~B})-\mathrm{C}(7 \mathrm{~B})-\mathrm{C}(8 \mathrm{~B}) & 121.37(11) \\ \mathrm{N}(1 \mathrm{~B})-\mathrm{C}(7 \mathrm{~B})-\mathrm{C}(8 \mathrm{~B}) & 126.47(11) \\ \mathrm{C}(13 \mathrm{~B})-\mathrm{C}(8 \mathrm{~B})-\mathrm{C}(9 \mathrm{~B}) & 116.77(12) \\ \mathrm{C}(13 \mathrm{~B})-\mathrm{C}(8 \mathrm{~B})-\mathrm{C}(7 \mathrm{~B}) & 126.57(11) \\ \mathrm{C}(9 \mathrm{~B})-\mathrm{C}(8 \mathrm{~B})-\mathrm{C}(7 \mathrm{~B}) & 116.38(11) \\ \mathrm{C}(10 \mathrm{~B})-\mathrm{C}(9 \mathrm{~B})-\mathrm{C}(8 \mathrm{~B}) & 121.97(13) \\ \mathrm{C}(10 \mathrm{~B})-\mathrm{C}(9 \mathrm{~B})-\mathrm{H}(9 \mathrm{~B}) & 119.0 \\ \mathrm{C}(8 \mathrm{~B})-\mathrm{C}(9 \mathrm{~B})-\mathrm{H}(9 \mathrm{~B}) & 119.0 \\ \mathrm{C}(9 \mathrm{~B})-\mathrm{C}(10 \mathrm{~B})-\mathrm{C}(11 \mathrm{~B}) & 119.98(13) \\ \mathrm{C}(9 \mathrm{~B})-\mathrm{C}(10 \mathrm{~B})-\mathrm{H}(10 \mathrm{~B}) & 120.0 \\ \mathrm{C}(11 \mathrm{~B})-\mathrm{C}(10 \mathrm{~B})-\mathrm{H}(10 \mathrm{~B}) & 120.0 \\ \mathrm{C}(12 \mathrm{~B})-\mathrm{C}(11 \mathrm{~B})-\mathrm{C}(10 \mathrm{~B}) & 119.42(13) \\ \mathrm{C}(12 \mathrm{~B})-\mathrm{C}(11 \mathrm{~B})-\mathrm{H}(11 \mathrm{~B}) & 120.3 \\ \mathrm{C}(10 \mathrm{~B})-\mathrm{C}(11 \mathrm{~B})-\mathrm{H}(11 \mathrm{~B} & 120.3 \\ \mathrm{C}(11 \mathrm{~B})-\mathrm{C}(12 \mathrm{~B})-\mathrm{C}(13 \mathrm{~B}) & 120.61(13) \\ \mathrm{C}(11 \mathrm{~B})-\mathrm{C}(12 \mathrm{~B})-\mathrm{H}(12 \mathrm{~B}) & 119.7 \\ \mathrm{C}(13 \mathrm{~B})-\mathrm{C}(12 \mathrm{~B})-\mathrm{H}(12 \mathrm{~B}) & 119.7 \\ \mathrm{O}(1 \mathrm{~B})-\mathrm{C}(13 \mathrm{~B})-\mathrm{C}(12 \mathrm{~B}) & 113.95(11) \\ \mathrm{O}(1 \mathrm{~B})-\mathrm{C}(13 \mathrm{~B})-\mathrm{C}(8 \mathrm{~B}) & 125.01(11) \\ \mathrm{C}(12 \mathrm{~B})-\mathrm{C}(13 \mathrm{~B})-\mathrm{C}(8 \mathrm{~B}) & \\ & \\ & \end{array}$




$\begin{array}{ll}\text { O(1B)-C(14B)-C(15B) } & 108.88(10) \\ \text { O(1B)-C(14B)-C(16B) } & 106.19(9) \\ \text { C(15B)-C(14B)-C(16B) } & 114.47(10) \\ \text { O(1B)-C(14B)-H(14B) } & 109.1 \\ \text { C(15B)-C(14B)-H(14B) } & 109.1 \\ \text { C(16B)-C(14B)-H(14B) } & 109.1 \\ \text { N(1B)-C(15B)-C(14B) } & 111.79(10) \\ \text { N(1B)-C(15B)-H(15C) } & 109.3 \\ \text { C(14B)-C(15B)-H(15C) } & 109.3 \\ \text { N(1B)-C(15B)-H(15D) } & 109.3 \\ \text { C(14B)-C(15B)-H(15D) } & 109.3 \\ \text { H(15C)-C(15B)-H(15D) } & 107.9 \\ \text { C(19B)-C(16B)-C(17B) } & 109.69(11) \\ \text { C(19B)-C(16B)-C(18B) } & 109.86(11) \\ \text { C(17B)-C(16B)-C(18B) } & 108.68(11) \\ \text { C(19B)-C(16B)-C(14B) } & 110.44(10) \\ \text { C(17B)-C(16B)-C(14B) } & 109.90(10) \\ \text { C(18B)-C(16B)-C(14B) } & 108.24(10) \\ \text { C(16B)-C(17B)-H(17D) } & 109.5 \\ \text { C(16B)-C(17B)-H(17E) } & 109.5 \\ \text { H(17D)-C(17B)-H(17E) } & 109.5 \\ \text { C(16B)-C(17B)-H(17F) } & 109.5 \\ \text { H(17D)-C(17B)-H(17F) } & 109.5 \\ \text { H(17E)-C(17B)-H(17F) } & 109.5 \\ \text { C(16B)-C(18B)-H(18D) } & 109.5 \\ \text { C(16B)-C(18B)-H(18E) } & 109.5 \\ \text { H(18D)-C(18B)-H(18E) } & 109.5 \\ \text { C(16B)-C(18B)-H(18F) } & 109.5 \\ \text { H(18D)-C(18B)-H(18F) } & 109.5 \\ \text { H(18E)-C(18B)-H(18F) } & 109.5 \\ \text { C(16B)-C(19B)-H(19D) } & 109.5 \\ & \end{array}$




$\begin{array}{ll}\text { C(16B)-C(19B)-H(19E) } & 109.5 \\ \text { H(19D)-C(19B)-H(19E) } & 109.5 \\ \text { C(16B)-C(19B)-H(19F) } & 109.5 \\ \text { H(19D)-C(19B)-H(19F) } & 109.5 \\ \text { H(19E)-C(19B)-H(19F) } & 109.5\end{array}$


Table S4. Anisotropic Displacement Parameters $\left(\AA^{2} \times \mathbf{1 0}^{3}\right)$ for Benzimidazole (9). ${ }^{19}$

\begin{tabular}{|c|c|c|c|c|c|c|}
\hline & U11 & $\mathrm{U} 22$ & U33 & $\mathrm{U} 23$ & U13 & U12 \\
\hline $\mathrm{O}(1 \mathrm{~A})$ & $30(1)$ & $35(1)$ & $37(1)$ & $-5(1)$ & $10(1)$ & $0(1)$ \\
\hline $\mathrm{N}(1 \mathrm{~A})$ & $30(1)$ & $33(1)$ & $30(1)$ & $-1(1)$ & $8(1)$ & $0(1)$ \\
\hline $\mathrm{N}(2 \mathrm{~A})$ & $35(1)$ & $39(1)$ & $33(1)$ & $0(1)$ & $6(1)$ & $-2(1)$ \\
\hline $\mathrm{C}(1 \mathrm{~A})$ & $35(1)$ & $28(1)$ & $31(1)$ & $0(1)$ & $10(1)$ & $4(1)$ \\
\hline$C(2 A)$ & $37(1)$ & $33(1)$ & $42(1)$ & $0(1)$ & $13(1)$ & $-1(1)$ \\
\hline$C(3 A)$ & $49(1)$ & $36(1)$ & $43(1)$ & $5(1)$ & $22(1)$ & $3(1)$ \\
\hline$C(4 A)$ & $57(1)$ & $38(1)$ & $33(1)$ & $2(1)$ & $15(1)$ & $4(1)$ \\
\hline$C(5 A)$ & $46(1)$ & $40(1)$ & $33(1)$ & $0(1)$ & $6(1)$ & $0(1)$ \\
\hline$C(6 A)$ & $36(1)$ & $31(1)$ & $34(1)$ & $1(1)$ & $9(1)$ & $2(1)$ \\
\hline$C(7 A)$ & $29(1)$ & $28(1)$ & $34(1)$ & $0(1)$ & $7(1)$ & $2(1)$ \\
\hline$C(8 A)$ & $28(1)$ & $29(1)$ & $36(1)$ & $2(1)$ & $9(1)$ & $4(1)$ \\
\hline$C(9 A)$ & $33(1)$ & $35(1)$ & $41(1)$ & $2(1)$ & $7(1)$ & $0(1)$ \\
\hline $\mathrm{C}(10 \mathrm{~A})$ & $33(1)$ & $37(1)$ & $56(1)$ & $7(1)$ & $13(1)$ & $-2(1)$ \\
\hline$C(11 \mathrm{~A})$ & $39(1)$ & $44(1)$ & $47(1)$ & $13(1)$ & $21(1)$ & $4(1)$ \\
\hline $\mathrm{C}(12 \mathrm{~A})$ & $37(1)$ & $39(1)$ & $37(1)$ & $5(1)$ & $13(1)$ & $5(1)$ \\
\hline$C(13 A)$ & $28(1)$ & $27(1)$ & $37(1)$ & $2(1)$ & $9(1)$ & $3(1)$ \\
\hline $\mathrm{C}(14 \mathrm{~A})$ & $29(1)$ & $29(1)$ & $35(1)$ & $-4(1)$ & $9(1)$ & $2(1)$ \\
\hline$C(15 A)$ & $30(1)$ & $39(1)$ & $35(1)$ & $-3(1)$ & $7(1)$ & $-3(1)$ \\
\hline$C(16 A)$ & $32(1)$ & $35(1)$ & $35(1)$ & $-4(1)$ & $4(1)$ & $1(1)$ \\
\hline $\mathrm{C}(17 \mathrm{~A})$ & $36(1)$ & $60(1)$ & $57(1)$ & $-16(1)$ & $-2(1)$ & $11(1)$ \\
\hline $\mathrm{C}(18 \mathrm{~A})$ & $52(1)$ & $46(1)$ & $39(1)$ & $6(1)$ & $-3(1)$ & $-8(1)$ \\
\hline$C(19 A)$ & $40(1)$ & $40(1)$ & $42(1)$ & $-7(1)$ & $10(1)$ & $-6(1)$ \\
\hline $\mathrm{O}(1 \mathrm{~B})$ & $34(1)$ & $44(1)$ & $47(1)$ & $-14(1)$ & $15(1)$ & $-4(1)$ \\
\hline $\mathrm{N}(1 \mathrm{~B})$ & $33(1)$ & $32(1)$ & $30(1)$ & $0(1)$ & $9(1)$ & $-1(1)$ \\
\hline $\mathrm{N}(2 \mathrm{~B})$ & $39(1)$ & $43(1)$ & $33(1)$ & $-6(1)$ & 11(1) & $-5(1)$ \\
\hline $\mathrm{C}(1 \mathrm{~B})$ & $31(1)$ & $38(1)$ & $32(1)$ & $5(1)$ & 11(1) & $-5(1)$ \\
\hline $\mathrm{C}(2 \mathrm{~B})$ & $39(1)$ & $41(1)$ & $43(1)$ & $6(1)$ & $13(1)$ & $0(1)$ \\
\hline
\end{tabular}

${ }^{19}$ The anisotropic displacement factor exponent takes the form:- $2 \pi^{2}\left[h^{2} a^{* 2} U 11+\ldots+2 h k a^{*} b^{*} U 12\right]$. 


\begin{tabular}{|c|c|c|c|c|c|c|}
\hline $\mathrm{C}(3 \mathrm{~B})$ & $38(1)$ & $52(1)$ & $50(1)$ & $15(1)$ & 11(1) & $1(1)$ \\
\hline $\mathrm{C}(4 \mathrm{~B})$ & $37(1)$ & $66(1)$ & $40(1)$ & 11(1) & $5(1)$ & $-9(1)$ \\
\hline $\mathrm{C}(5 \mathrm{~B})$ & $42(1)$ & $56(1)$ & $37(1)$ & $-2(1)$ & $9(1)$ & $-12(1)$ \\
\hline $\mathrm{C}(6 \mathrm{~B})$ & $33(1)$ & $43(1)$ & $33(1)$ & $1(1)$ & $12(1)$ & $-7(1)$ \\
\hline$C(7 B)$ & $35(1)$ & $32(1)$ & $30(1)$ & $-1(1)$ & $14(1)$ & $-5(1)$ \\
\hline $\mathrm{C}(8 \mathrm{~B})$ & $39(1)$ & $30(1)$ & $32(1)$ & $3(1)$ & $16(1)$ & $-1(1)$ \\
\hline $\mathrm{C}(9 \mathrm{~B})$ & $51(1)$ & $34(1)$ & $35(1)$ & $0(1)$ & $18(1)$ & $2(1)$ \\
\hline $\mathrm{C}(10 \mathrm{~B})$ & $62(1)$ & $37(1)$ & $48(1)$ & $5(1)$ & $27(1)$ & $15(1)$ \\
\hline $\mathrm{C}(11 \mathrm{~B})$ & $49(1)$ & $49(1)$ & $46(1)$ & $11(1)$ & $17(1)$ & $17(1)$ \\
\hline $\mathrm{C}(12 \mathrm{~B})$ & $40(1)$ & $42(1)$ & $38(1)$ & $7(1)$ & $10(1)$ & $5(1)$ \\
\hline $\mathrm{C}(13 \mathrm{~B})$ & $38(1)$ & $30(1)$ & $34(1)$ & $3(1)$ & $16(1)$ & $-1(1)$ \\
\hline $\mathrm{C}(14 \mathrm{~B})$ & $31(1)$ & $29(1)$ & $33(1)$ & $-1(1)$ & $11(1)$ & $-2(1)$ \\
\hline $\mathrm{C}(15 \mathrm{~B})$ & $48(1)$ & $32(1)$ & $32(1)$ & $-3(1)$ & $9(1)$ & $1(1)$ \\
\hline$C(16 B)$ & $40(1)$ & $30(1)$ & $29(1)$ & $-2(1)$ & $8(1)$ & $1(1)$ \\
\hline $\mathrm{C}(17 \mathrm{~B})$ & $52(1)$ & $41(1)$ & $37(1)$ & $0(1)$ & $18(1)$ & $4(1)$ \\
\hline $\mathrm{C}(18 \mathrm{~B})$ & $54(1)$ & $41(1)$ & $35(1)$ & $2(1)$ & $7(1)$ & $6(1)$ \\
\hline $\mathrm{C}(19 \mathrm{~B})$ & $49(1)$ & $36(1)$ & $36(1)$ & $-6(1)$ & $6(1)$ & $-4(1)$ \\
\hline
\end{tabular}


Table S5. Calculated Hydrogen Coordinates $\left[(x, y, z), \times 10^{4}\right]$ and Isotropic Displacement Parameters U(eq) $\left(\AA^{2} \times \mathbf{1 0}^{3}\right)$ for Benzimidazole $(9)$.

\begin{tabular}{|c|c|c|c|c|}
\hline & $\mathrm{x}$ & $\mathrm{y}$ & $\mathrm{z}$ & $\mathrm{U}(\mathrm{eq})$ \\
\hline $\mathrm{H}(2 \mathrm{~A})$ & $1384(1)$ & $6300(1)$ & $3156(1)$ & 44 \\
\hline $\mathrm{H}(3 \mathrm{~A})$ & $1650(1)$ & $6328(1)$ & $4153(1)$ & 49 \\
\hline $\mathrm{H}(4 \mathrm{~A})$ & $3190(1)$ & $7203(1)$ & $4751(1)$ & 50 \\
\hline $\mathrm{H}(5 \mathrm{~A})$ & $4528(1)$ & $8065(1)$ & $4381(1)$ & 49 \\
\hline $\mathrm{H}(9 \mathrm{~A})$ & $5440(1)$ & $9617(1)$ & $2735(1)$ & 44 \\
\hline $\mathrm{H}(10 \mathrm{~A})$ & $6296(1)$ & $10284(1)$ & $2053(1)$ & 50 \\
\hline $\mathrm{H}(11 \mathrm{~A})$ & $5722(1)$ & $9490(1)$ & $1115(1)$ & 50 \\
\hline $\mathrm{H}(12 \mathrm{~A})$ & $4220(1)$ & $8155(1)$ & $851(1)$ & 44 \\
\hline $\mathrm{H}(14 \mathrm{~A})$ & 2193(1) & $8752(1)$ & $1696(1)$ & 37 \\
\hline $\mathrm{H}(15 \mathrm{~A})$ & $1346(1)$ & $7174(1)$ & 2199(1) & 42 \\
\hline $\mathrm{H}(15 \mathrm{~B})$ & $2114(1)$ & $6055(1)$ & $2058(1)$ & 42 \\
\hline $\mathrm{H}(17 \mathrm{~A})$ & $6(5)$ & $7831(6)$ & $1532(3)$ & 81 \\
\hline $\mathrm{H}(17 \mathrm{~B})$ & $-416(3)$ & $8293(9)$ & $878(1)$ & 81 \\
\hline $\mathrm{H}(17 \mathrm{C})$ & $372(3)$ & $9232(3)$ & 1331(4) & 81 \\
\hline $\mathrm{H}(18 \mathrm{~A})$ & $1959(5)$ & $7886(6)$ & $459(3)$ & 73 \\
\hline $\mathrm{H}(18 \mathrm{~B})$ & $1569(7)$ & $9266(3)$ & $666(2)$ & 73 \\
\hline $\mathrm{H}(18 \mathrm{C})$ & $742(3)$ & $8316(9)$ & $239(1)$ & 73 \\
\hline $\mathrm{H}(19 \mathrm{~A})$ & $667(6)$ & $5727(2)$ & $1241(2)$ & 61 \\
\hline $\mathrm{H}(19 \mathrm{~B})$ & $1522(2)$ & $5716(2)$ & $874(4)$ & 61 \\
\hline $\mathrm{H}(19 \mathrm{C})$ & $317(5)$ & $6098(1)$ & $575(2)$ & 61 \\
\hline $\mathrm{H}(2 \mathrm{~B})$ & $3031(1)$ & $5790(1)$ & $365(1)$ & 48 \\
\hline $\mathrm{H}(3 \mathrm{~B})$ & $1592(1)$ & $5738(2)$ & $-450(1)$ & 56 \\
\hline $\mathrm{H}(4 \mathrm{~B})$ & $1454(1)$ & $4089(2)$ & $-1141(1)$ & 59 \\
\hline $\mathrm{H}(5 \mathrm{~B})$ & $2728(1)$ & $2422(2)$ & $-1046(1)$ & 54 \\
\hline $\mathrm{H}(9 \mathrm{~B})$ & $5971(1)$ & $672(1)$ & $64(1)$ & 46 \\
\hline $\mathrm{H}(10 \mathrm{~B})$ & $7664(1)$ & $-182(1)$ & $360(1)$ & 56 \\
\hline $\mathrm{H}(11 \mathrm{~B})$ & $8922(1)$ & $843(1)$ & $1108(1)$ & 56 \\
\hline
\end{tabular}




$\begin{array}{lrrrr}\mathrm{H}(12 B) & 8409(1) & 2588(1) & 1609(1) & 48 \\ \mathrm{H}(14 B) & 5328(1) & 2962(1) & 1564(1) & 37 \\ \mathrm{H}(15 \mathrm{C}) & 4344(1) & 4987(1) & 1176(1) & 45 \\ \mathrm{H}(15 \mathrm{D}) & 5385(1) & 5445(1) & 997(1) & 45 \\ \mathrm{H}(17 \mathrm{D}) & 4342(3) & 5423(6) & 2008(2) & 63 \\ \mathrm{H}(17 \mathrm{E}) & 4854(1) & 5086(8) & 2669(1) & 63 \\ \mathrm{H}(17 \mathrm{~F}) & 4344(3) & 3920(2) & 2232(4) & 63 \\ \mathrm{H}(18 \mathrm{D}) & 6587(6) & 3969(4) & 2982(1) & 66 \\ \mathrm{H}(18 \mathrm{E}) & 7145(3) & 3380(7) & 2520(2) & 66 \\ \mathrm{H}(18 \mathrm{~F}) & 6074(3) & 2724(4) & 2594(3) & 66 \\ \mathrm{H}(19 \mathrm{D}) & 5966(3) & 6478(3) & 1903(3) & 62 \\ \mathrm{H}(19 \mathrm{E}) & 7072(3) & 5700(2) & 2068(4) & 62 \\ \mathrm{H}(19 \mathrm{~F}) & 6558(6) & 6214(4) & 2561(1) & 62\end{array}$

